Y-12

NATIONAL SECURITY COMPLEX

\section{Annual Storm Water Report for the \\ Y-12 National Security Complex Oak Ridge, Tennessee} \section{December 2013}

\author{
Prepared by the \\ Clean Water Compliance Section \\ of the
}

Environment Compliance Department Environment, Safety and Health

Prepared for the Y-12 National Security Complex Oak Ridge, Tennessee 37831 managed by B\&W Technical Services Y-12, L.L.C. for the

U.S. DEPARTMENT OF ENERGY

under contract

DE-AC05-00OR22800 


\section{Disclaimer}

This report was prepared as an account of work sponsored by an agency of the United States Government. Neither the United States Government nor any agency thereof, nor any of their employees, makes any warranty, express or implied, or assumes any legal liability or responsibility for the accuracy, completeness, or usefulness of any information, apparatus, product, or process disclosed, or represents that its use would not infringe privately owned rights. Reference herein to any specific commercial product, process, or service by trade name, trademark, manufacturer, or otherwise, does not necessarily constitute or imply its endorsement, recommendation, or favoring by the United States Government or any agency thereof. The views and opinions of authors expressed herein do not necessarily state or reflect those of the United States Government or any agency thereof. 


\title{
Annual Storm Water Report for the Y-12 National Security Complex Oak Ridge, Tennessee
}

\section{December 2013}

\author{
Prepared by the \\ Clean Water Compliance Section \\ of the \\ Environmental Compliance Department \\ Environment, Safety and health
}

Prepared for the

Y-12 National Security Complex

Oak Ridge, Tennessee 37831

managed by

B\&W Technical Services Y-12, L.L.C.

for the

U.S. DEPARTMENT OF ENERGY

under contract

DE-AC05-00OR22800 


\section{Table of Contents}

List of Tables and Figure

Page

iv

Executive Summary

$\mathrm{v}$

1.0 Introduction

2.0 Storm Water Monitoring Data

2.1 Sector Outfalls

2.1.1 Sector AA - Storm Water Discharges Associated with Industrial Activity from Fabricated Metal Products Industry

2.1.2 Sector N - Storm Water Discharges Associated with Industrial Activity from Scrap Recycling and Waste Recycling Facilities

2.1.3 Sector L - Storm Water Discharges Associated with Industrial Activity from Landfills and Land Application Sites

2.1.4 Sector K - Storm Water Discharges Associated with Treatment, Storage and Disposal Facilities

2.2 In-Stream Monitoring Locations and Major Outfalls

3.0 Surveillance Results and Significant Projects

4.0 Conclusion.

Appendix A. Schematic of Storm Water Outfall Analytical Data on EFPC, 2006 - 2013 ............... 8

Appendix B. 2013 Storm Water Analytical Results, Sector Outfalls ....................................... 17

Appendix C. 20/3 Storm Water Analytical Results, In-Stream Monitoring Locations and Major Outfalls. 


\section{List of Tables}

Table 2.1 Sector AA, Fabricated Metal Products Industry Analytical Results ............................... 2

Table 2.2 Sector N, Scrap Recycling and Waste Recycling Facilities Analytical Results.................. 3

Table 2.3 Sector L, Landfills and Land Application Sites Analytical Results ............................. 4

Table 2.4 Sector K, Treatment, Storage and Disposal Facilities Analytical Results........................ 4

Table 2.5 Flows and Flow Rates at In-Stream Monitoring Points on EFPC

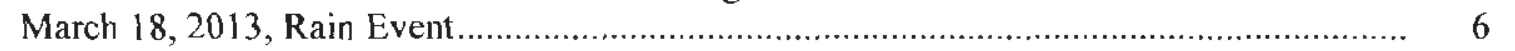

Table 2.6 Flows and Flow Rates at Major Outfalls and Raw Water on EFPC

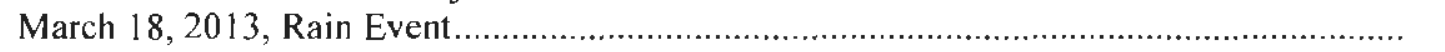

\section{Figure}

Figure $\quad$ Y-12 Complex Monitoring of East Fork Poplar Creek .......................................... 5 


\section{Executive Summary}

This is the second annual storm water report prepared in accordance with the National Pollutant Discharge Elimination System (NPDES) permit issued to the Y-12 National Security Complex (Y-12 Complex) on December 1, 2011, and the corresponding Y-12 Storm Water Pollution Prevention Plan (SWP3) which became effective on September 7, 2012. However, Appendix A does contain some analytical data gathered under the previous NPDES permit and SWP3 for comparison purposes.

The quality of storm water exiting the Y-12 Complex via East Fork Poplar Creek remained relatively stable from 2012 to 2013 . However, there was one largely unexpected high concentration of mercury noted in an area that is not known to have previously been a mercury use area. This was noted in Sector AA, Outfall 014. This outfall is normally sampled on a rotating basis but, due this elevated concentration, will be sampled again in 2014 .

The Y-12 Complex will continue to implement appropriate BMPs and reduce outside material storage ares where possible. Emphasis will continue to be placed on site inspections and timely implementation of proper storm water control measures. 


\subsection{Introduction}

The storm water pollution prevention program at the $Y-12$ National Security Complex (Y-12 Complex) protects the quality of storm water runoff through: (1) reducing the exposure of metal accumulation areas to precipitation, (2) implementation of Best Management Practices (BMPs), (3) sampling during rain events and subsequent analysis, and (4) routine surveillances. The Y-12 Complex was issued a new National Pollutant Discharge Elimination System (NPDES) permit which became effective on December 1,2011. Although this report is prepared in accordance with the most recent NPDES permit, it also contains some data collected in previous years for comparison purposes.

The storm water pollution prevention program is a subset of the Y-12 Environmental Management System (EMS) which was fully implemented at the Y-12 Complex in December 2005. The storm water program focuses primarily on two elements of the EMS policy: pollution prevention and continual improvement. Additionally, the quality of storm water that exits the Y-12 Complex is identified as a significant environmental aspect which requires adequate attention be given to this ongoing program.

\subsection{Storm Water Monitoring Data}

Per the NPDES permit, storm water monitoring at the Y-12 Complex is performed on three levels: sector outfalls, in-stream monitoring locations and major outfalls. Sector outfall monitoring is designed to detect specific pollutants commonly associated with various industrial activities. In-stream and major outfall monitoring are intended to provide an indication of the storm water quality that leaves the Y-12 Complex via East Fork Poplar Creek (EFPC).

\subsection{Sector Outfalls}

Although the NPDES permit defines the Y-12 Complex to be a fabricated metal products industry, it also requires storm water monitoring be conducted for three additional "sectors." These sectors are defined in the Tennessee Storm Water Multi-Sector General Permit for Industrial Activities, Permit No. TNR050000. Each sector has prescribed benchmark values and some have defined sector median values. The Rationale portion of the NPDES permit for the Y-12 Complex states ". . benchmark values were developed by the EPA and the State of Tennessee and are based on data submitted by similar industries for the development of the multisector general storm water permit. The benchmark concentrations are target values and should not be construed to represent permit limits." Similarly, sector median values are defined in the Tennessee Storm Water Multi-Sector General Permit as ". . a pollutant concentration calculated from all sampling results provided from facilities classified in this sector during the previous permit term." The following subsections summarize the sector monitoring activities and results during 2013. All sector outfall samples are collected via grab samples during the first 30 minutes of outfall discharge attributable to a storm event.

The complete set of data for all sector outfalls is contained in Appendix B. Although the NPDES permit requires these samples to be collected by the grab method, a composite sample for most of these outfalls was also collected, analyzed and reported. The analytical results from the composite samples can also be found in Appendix B. 


\subsubsection{Sector AA - Storm Water Discharges Associated with Ind ustrial Activity from Fabricated Metal Products Ind ustry}

The Storm Water Pollution Prevention Plan for the Y-12 Complex identifies six outfalls in this sector and each outfall is required to be sampled only once during the life of the NPDES permit.

The samples for Outfalls 014,067 and 102 were collected on the dates identified in Table 2.1. This table also contains the total rainfall and duration of the rain event. The grab samples were collected during the first 30 minutes of increased flow due to the rain event and there were more than 72 hours of dry weather prior to each rain event.

Table 2.1 - Sector AA, Fabricated Metal Products Industry Analytical Results - Grab Samples (All values are mg/L)

\begin{tabular}{||c|c|c|c|c|}
\hline Parameter & $\begin{array}{c}\text { Benchmark } \\
\text { Value }\end{array}$ & $\begin{array}{c}\text { Outfall 014 } \\
\text { Sampled } \\
\text { April 24, 2013 }\end{array}$ & $\begin{array}{c}\text { Outfall 067 } \\
\text { Sampled } \\
\text { April 4,2013 }\end{array}$ & $\begin{array}{c}\text { Outfall 102 } \\
\text { Sampled } \\
\text { April 4, 2013 }\end{array}$ \\
\hline $\begin{array}{c}\text { Total Rainfall and } \\
\text { Duration }\end{array}$ & N/A & $\begin{array}{c}0.15 \text { inches } \\
65 \text { minutes }\end{array}$ & $\begin{array}{c}0.22 \text { inches } \\
115 \text { minutes }\end{array}$ & $\begin{array}{c}0.24 \text { inches } \\
185 \text { minutes }\end{array}$ \\
\hline $\begin{array}{c}\text { Total Recoverable } \\
\text { Aluminum }\end{array}$ & 0.75 & 0.533 & $<0.2$ & $<0.2$ \\
\hline $\begin{array}{c}\text { Total Recoverable } \\
\text { Iron }\end{array}$ & 5.0 & 1.75 & 0.209 & 0.17 \\
\hline $\begin{array}{c}\text { Total Recoverable } \\
\text { Zinc }\end{array}$ & 0.161 & 0.0552 & 0.0592 & 0.0986 \\
\hline $\begin{array}{c}\text { Nitrate plus Nitrite } \\
\text { Nitrogen }\end{array}$ & 0.68 & 0.583 & 0.67 & 0.351 \\
\hline \hline
\end{tabular}

All parameters at Outfalls 014,067 and 102 were below the benchmark values. However, additional parameters beyond the benchmark parameters are routinely analyzed and the concentration of mercury at Outfall 014 was unusually high at $0.00712 \mathrm{mg} / \mathrm{L}$. This was unexpected as this outfall drains an area which has no history of mercury use. For this reason, this outfall will be resampled next year even though it is scheduled to be off the rotation with other Sector AA outfalls.

\subsubsection{Sector N - Storm Water Discharges Associated with Industrial Activity from Scrap Recycling and Waste Recycling Facilities}

The NPDES permit for the Y-12 Complex defines only one outfall (Outfall S30) in this sector. This outfall was created specifically to monitor storm water runoff from the New Salvage Yard located west of the main industrial complex. This outfall does not discharge directly into the waters of the state (Bear Creek). The discharge from this outfall sheet flows across approximately 50 feet of grassy terrain prior to reaching Bear Creek. Therefore, concentrations of pollutants that may enter the creek can be expected to be lower than measured at the monitoring point. This outfall is sampled annually. Table 2.2 presents the benchmark values, the sector median values and the analytical results for each of the prescribed parameters for this sector. 
The samples for Outfall S30 were collected on September 21, 2013. This rain event lasted 182 minutes and had a total rainfall of 1.05 inches. The grab samples were collected during the first 30 minutes of flow from the discharge pipe. There were more than 72 hours of prior dry weather.

Table 2.2 - Sector N, Scrap Recycling and Waste Recycling Facilities Analytical Results - Grab Samples (All values are mg/L)

\begin{tabular}{|c|c|c|c|}
\hline Parameter & $\begin{array}{c}\text { Benchmark } \\
\text { Value }\end{array}$ & $\begin{array}{c}\text { Sector Median } \\
\text { Value }\end{array}$ & $\begin{array}{c}\text { Outfall S30 } \\
\text { Sampled } \\
\text { September 21,2013 }\end{array}$ \\
\hline $\begin{array}{c}\text { Chemical Oxygen } \\
\text { Demand (COD) }\end{array}$ & 120 & 79 & 41.0 \\
\hline Total Suspended Solids & 150 & 72 & 13.5 \\
\hline $\begin{array}{c}\text { Total Recoverable } \\
\text { Aluminum }\end{array}$ & 0.75 & 2.08 & 0.878 \\
\hline Total Recoverable Copper & 0.0014 & 0.091 & 0.00724 \\
\hline Total Recoverable lron & 5.0 & 3.7 & 0.545 \\
\hline Total Recoverable Lead & 0.091 & 0.058 & 0.00364 \\
\hline Total Recoverable Zinc & 0.161 & 0.243 & 0.0146 \\
\hline
\end{tabular}

The aluminum and copper levels exceeded the benchmark values for this sector. However, both were below the sector median values, so this location is ranked in the bottom half of all locations which fall into this sector in the State of Tennessee. Aluminum levels continue to decline and are well below previous years' results $(1.51 \mathrm{mg} / \mathrm{L}$ for $2012,12.4 \mathrm{mg} / \mathrm{L}$ for 2011 and $10.8 \mathrm{mg} / \mathrm{L}$ for 2010 ) as a result of recent cleanup activities in this area. The levels of copper are consistent with the results from prior years; however, the benchmark value was reduced from $0.0636 \mathrm{mg} / \mathrm{L}$ in the previous permit to $0.0014 \mathrm{mg} / \mathrm{L}$ in the new permit. Had this significant reduction in the benchmark value not been made, this parameter would have been below the benchmark value.

\subsubsection{Sector L - Storm Water Discharges Associated with Industrial Activity from Landfills and Land Application Sites}

The NPDES permit for the Y-12 Complex defines two outfalls in this sector: Outfalls S17 and S18. Only one outfall is required to be sampled each year and the outfalls are alternated from year to year. Outfall S17 was sampled this year. Table 2.3 presents the benchmark values, the sector median values and the analytical results for the two defined parameters for this sector.

The samples for Outfall S17 were collected on April 4, 2013. At this location, the rain event lasted 120 minutes and had a total rainfall of 0.18 inches. The grab samples were collected during the first 30 minutes of discharge and there were more than 72 hours of dry weather prior to the onset of the rain event. 
Table 2.3 - Sector L, Land fills and Land Application Sites

Analytical Results - Grab Samples (All values are mg/L)

\begin{tabular}{|c|c|c|c|}
\hline Parameter & $\begin{array}{c}\text { Benchmark } \\
\text { Value }\end{array}$ & $\begin{array}{c}\text { Sector Median } \\
\text { Value }\end{array}$ & $\begin{array}{c}\text { Outfall S17 } \\
\text { Sampled } \\
\text { April 4, 2013 }\end{array}$ \\
\hline $\begin{array}{c}\text { Total Suspended } \\
\text { Solids (TSS) }\end{array}$ & 150 & 47 & 3.5 \\
\hline $\begin{array}{c}\text { Total } \\
\text { Recoverable Iron }\end{array}$ & 5.0 & 2.2 & 0.103 \\
\hline
\end{tabular}

Both parameters at Outfall S17 were below the benchmark and sector median values.

\subsubsection{Sector K - Storm Water Discharges Associated with Treatment, Storage and Disposal Facilities}

The NPDES permit for the Y-12 Complex defines only one outfall in this sector: Outfall S06. This is an in-stream monitoring point located near the headwaters of Bear Creek, just downstream from several waste treatment facilities. This outfall is sampled annually. Table 2.4 presents the benchmark values, the sector median values and the analytical results for each of the prescribed parameters for this sector. The complete list of analytical results can be found in Appendix B.

The samples for Outfall S06 were collected on April 24, 2013. This rain event lasted 105 minutes and had a total rainfall of 0.16 inches. The grab samples were collected during the first 30 minutes of increased flow due to the rain event. There were more than 72 hours of dry weather prior to the onset of the rain event.

Table 2.4 - Sector K, Treatment, Storage and Disposal Facilities Analytical Results - Grab Samples (All values are mg/L)

\begin{tabular}{|c|c|c|c|}
\hline Parameter & $\begin{array}{c}\text { Benchmark } \\
\text { Value }\end{array}$ & $\begin{array}{c}\text { Sector Median } \\
\text { Value }\end{array}$ & $\begin{array}{c}\text { Outfall S06 } \\
\text { Sampled } \\
\text { April 24, 2013 }\end{array}$ \\
\hline $\begin{array}{c}\text { Ammonia } \\
\text { Total Recoverable } \\
\text { Magnesium }\end{array}$ & 4.0 & 0.21 & $<0.2$ \\
\hline $\begin{array}{c}\text { Chemical Oxygen } \\
\text { Demand (COD) }\end{array}$ & 120 & 1.41 & 5.0 \\
\hline $\begin{array}{c}\text { Total Recoverable } \\
\text { Cadmium }\end{array}$ & 0.0021 & 20 & 0.00188 \\
\hline $\begin{array}{c}\text { Total Cyanide* } \\
\text { Total Recoverable Lead }\end{array}$ & 0.022 & 0.010 & $<0.05$ \\
\hline $\begin{array}{c}\text { Total Recoverable } \\
\text { Mercury }\end{array}$ & 0.0014 & 0.016 & 0.0001 \\
\hline $\begin{array}{c}\text { Total Recoverable } \\
\text { Selenium }\end{array}$ & 0.005 & 0.0002 & $<0.004$ \\
\hline Total Suspended Solids & 150 & 0.100 & 7.5 \\
\hline Total Recoverable Silver & 0.0038 & 0.005 & $<0.0002$ \\
\hline \hline
\end{tabular}


* The detection level for this parameter is higher than normal as a consequence of the dilution factor (10) that had to be applied due to the nitrate level of $27.2 \mathrm{mg} / \mathrm{L}$. The elevated nitrates are a result of the proximity of this location to the S-3 Ponds.

The level of magnesium at this monitoring point significantly exceeds both the benchmark value and the sector mean value. However, the geology of this portion of the Tennessee valley typically results in abnormally high levels of magnesium. For example, the level of magnesium in the raw water from the Clinch River used to supplement the flow in EFPC was $10.3 \mathrm{mg} / \mathrm{L}$ on March 18. 2013.

It is indeterminable if the level of cyanide was within the prescribed cut-off concentrations due to the high detection limit. However, it is not expected that cyanide is a problem in this area due to the lack of this material in the upstream waste treatment facility and no historical evidence of cyanide in this portion of Bear Creek.

\subsection{In-Stream Monitoring Locations and Major Outfalls}

Two in-stream monitoring locations are listed in the NPDES permit and the SWP3. Both locations are in EFPC and designated as $\mathrm{C} 03$ and $\mathrm{C} 11$. Each is required to be sampled annually for an extensive list of parameters. Samples are taken via both the composite and grab methods.

The NPDES permit and SWP3 also call out three major outfalls $(021,109$ and 200) and the raw water flow augmentation for specific sampling. These three outfalls discharge approximately 85 percent of all storm water into EFPC from the industrialized portion of the $\mathrm{Y}-12$ Complex. The rate of raw water flow is a consistent 3.6 million gallons per day and not influenced by storm events. This discharge is sampled to establish a baseline of water quality coming from the Clinch River.

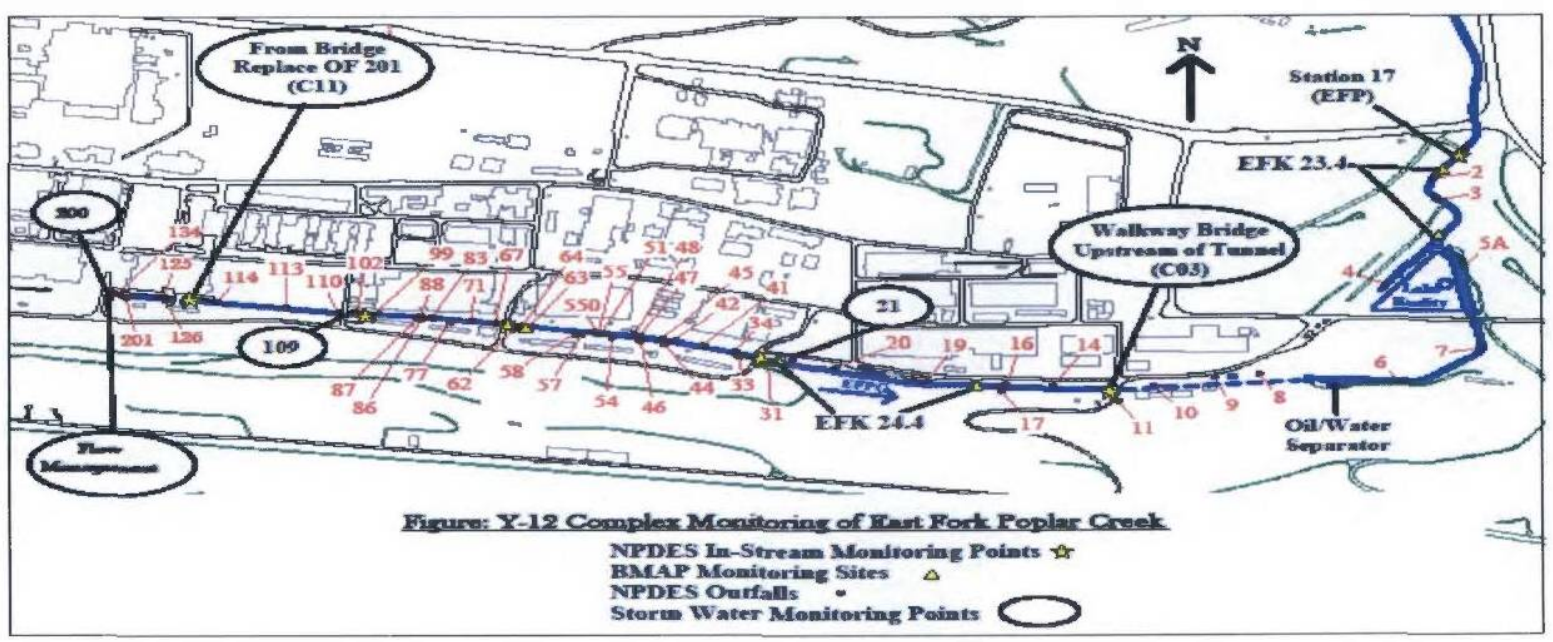

The figure above shows the locations of the in-stream and major outfalls. In conjunction with the diagrams in Appendix A, this figure depicts the major storm water monitoring locations on EFPC. 
On March 18, 2013, samples from the two in-stream monitoring points, three major outfalls and raw water were collected during the same rain event. This rain event began at 11:30 and ended at approximately 13:45. Total rainfall was 0.20 inches as measured at the meteorological tower located near the center of the Y-12 Complex; it had been more than 72 hours since the previous rain event of 0.10 inches or more. Tables 2.5 and 2.6 give synopses of flows and flow rates for the in-stream monitoring locations and major outfalls on EFPC during this rain event.

\section{Table 2.5 - Flows and Flow Rates at In-Stream Monitoring Points on EFPC March 18, 2013, Rain Event}

\begin{tabular}{|c|c|c|c|c|}
\hline $\begin{array}{c}\text { Sampling } \\
\text { Location }\end{array}$ & $\begin{array}{c}\text { Base (non-Storm) } \\
\text { Flow Rate (gpm) }\end{array}$ & $\begin{array}{c}\text { Maximum } \\
\text { Flow Rate } \\
(\mathrm{gpm})\end{array}$ & $\begin{array}{c}\text { Total Flow } \\
\text { (gallons) }\end{array}$ & $\begin{array}{c}\text { Duration of flow } \\
\text { Measurement (minutes) }\end{array}$ \\
\hline $\mathrm{C} 03$ & 3,300 & 11,177 & 989,500 & 140 \\
\hline $\mathrm{C} 11$ & 3,300 & 10,000 & $1,317,320$ & 175 \\
\hline
\end{tabular}

\section{Table 2.6 - Flows and Flow Rates at Major Outfalls and Raw Water on EFPC March 18, 2013, Rain Event}

\begin{tabular}{|c|c|c|c|c|}
\hline $\begin{array}{c}\text { Sampling } \\
\text { Location }\end{array}$ & $\begin{array}{c}\text { Base (non-Storm) } \\
\text { Flow Rate (gpm) }\end{array}$ & $\begin{array}{c}\text { Maximum } \\
\text { Flow Rate } \\
(\mathrm{gpm})\end{array}$ & $\begin{array}{c}\text { Total Flow } \\
\text { (gallons) }\end{array}$ & $\begin{array}{c}\text { Duration of flow } \\
\text { Measurement (minutes) }\end{array}$ \\
\hline 021 & 81 & $\mathbf{8 2 8}$ & 41,540 & 147 \\
\hline 109 & 41 & 2,788 & $\mathbf{8 4 , 3 5 7}$ & 138 \\
\hline $200^{*}$ & 488 & 7,327 & 903,141 & 132 \\
\hline Raw Water & 2,289 & 2,304 & 390,276 & 134 \\
\hline
\end{tabular}

* Flow at this location is a calculated value.

The first schematic in Appendix A is a pictorial depiction of discharge and monitoring points on EFPC. Also included are the analytical results for some of the more common storm water contaminants for each location. Nine locations are shown on this schematic, including the two instream monitoring locations, three major outfalls, raw water discharge and three Sector AA outfalls. Schematics for locations sampled during 2006 through 2011 are also included in Appendix A for comparison purposes.

The NPDES permit and the Y-12 SWP3 include an extensive list of parameters that are required to be analyzed for both the in-stream and major outfall locations. This data is included in Appendix C. Not all of this data could be included in the schematics in Appendix A due to the volume.

There are no benchmark or median values attributed to the in-stream monitoring locations. The data indicates a relatively consistent level of water quality for 2012 to 2013 . However, it is noteworthy that the concentration of mercury at in-stream Location $\mathrm{C} 03$ was reduced by approximately 44 percent from 2012 to 2013 . 


\subsection{Surveillance Results and Significant Projects}

The surveillances conducted during 2013 resulted in fewer and smaller areas identified as needing improvement. Most issues involved improper storage of drums, excess raw materials being stored outside and general housekeeping. Building and area owners were contacted and instructed to improve or reduce the outdoor storage of these items.

During 2013, the Y-12 Complex did not conduct any building demolitions. Over the past several years, numerous demolition projects were completed which provided a challenge in preventing contaminants and debris from entering EFPC.

There was one main project completed in 2013 which is expected to have a direct impact on the amount of mercury transported off the Y-12 Complex during storm events. A total of nine mercury traps have been installed in the storm drain system throughout the protected area of the Y-12 Complex. Dedicated mercury removal equipment has been procured and the removal process has been refined and implemented.

Other smaller projects focused on the reduction of outside storage areas and the removal of excess materials. At Building 9201-1, a project of note was the removal of metallic raw materials stored on the east side and large graphite billets stored on the west side. These materials were previously unprotected from contact with storm water and the areas drained to Outfalls 067 and 102, respectively. Outfalls 067 and 102 are Sector AA outfalls which are sampled on a rotating basis.

\subsection{Conclusion}

The data contained in Appendix A, which indicates the quality of storm water exiting the Y-12 Complex via EFPC, has remained relatively consistent from 2012 to 2013 . Y-12 Complex personnel will continue to implement appropriate BMPs and reduce outside material storage areas whenever possible. Emphasis will continue to be placed on site inspections and timely implementation of proper storm water control measures. 


\section{Appendix A \\ East Fork Poplar Creek Schematics}

2006 - 2013 
Schematic of Storm Water Outfall Anglytical Data on FFPC

2013

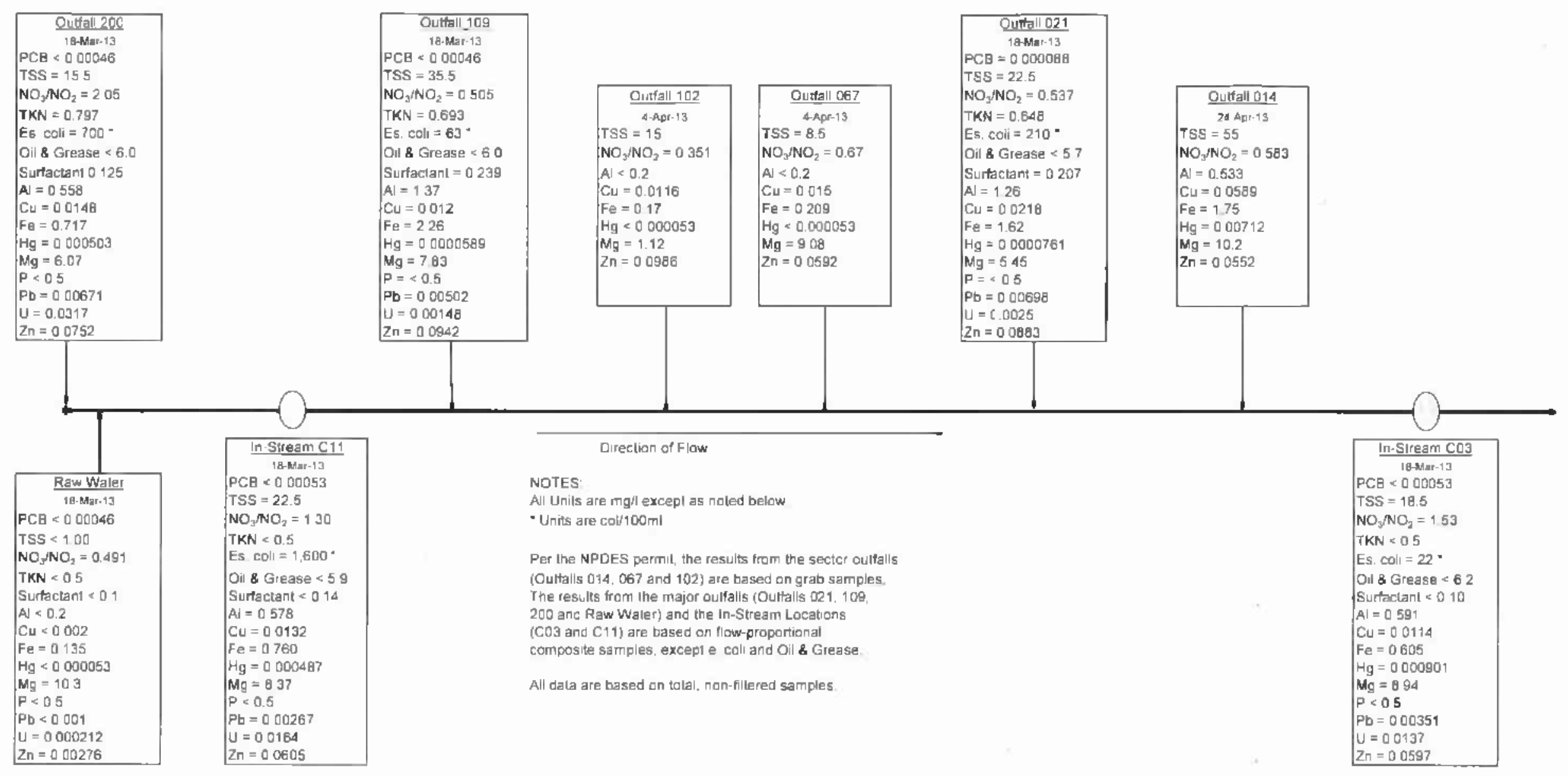




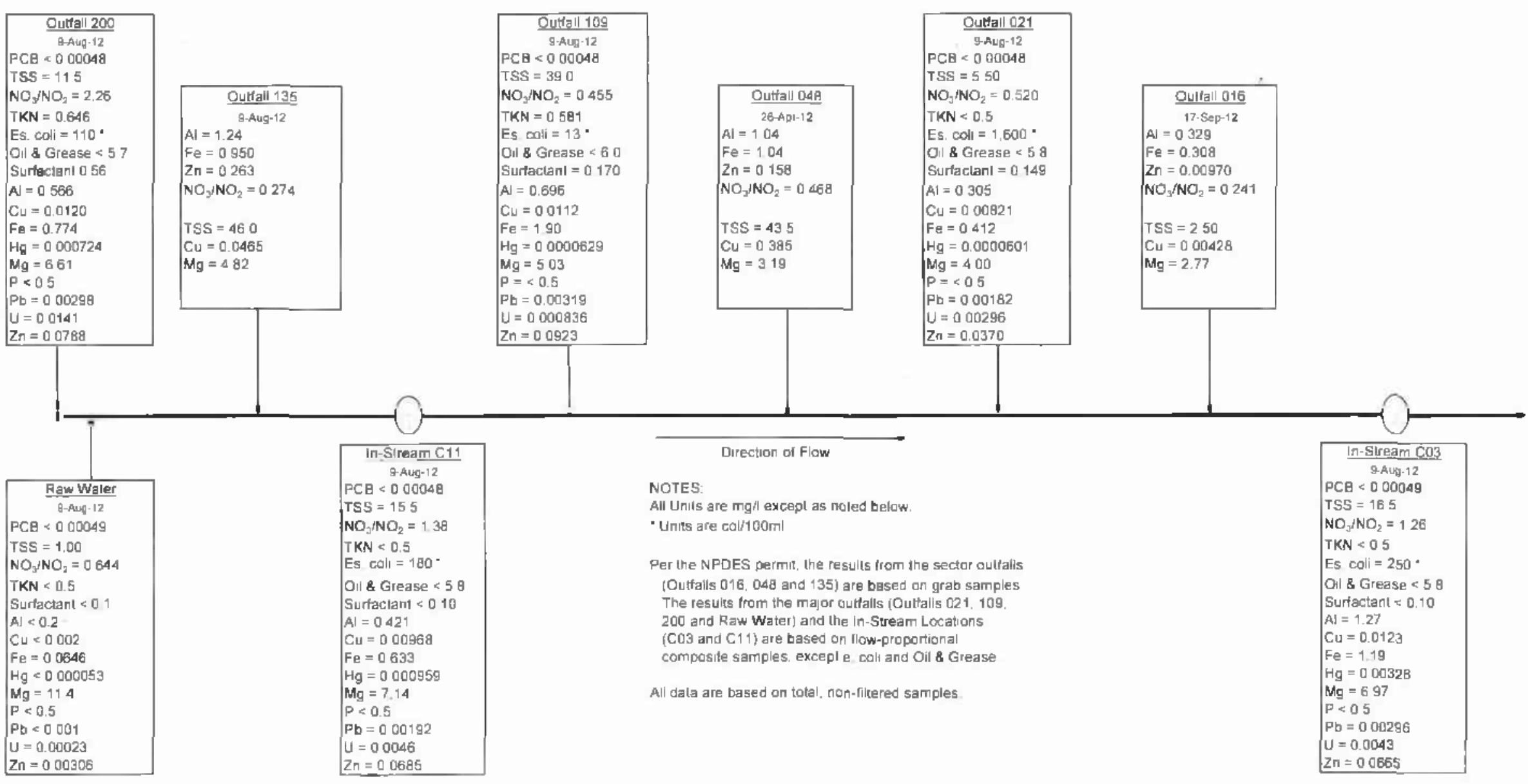




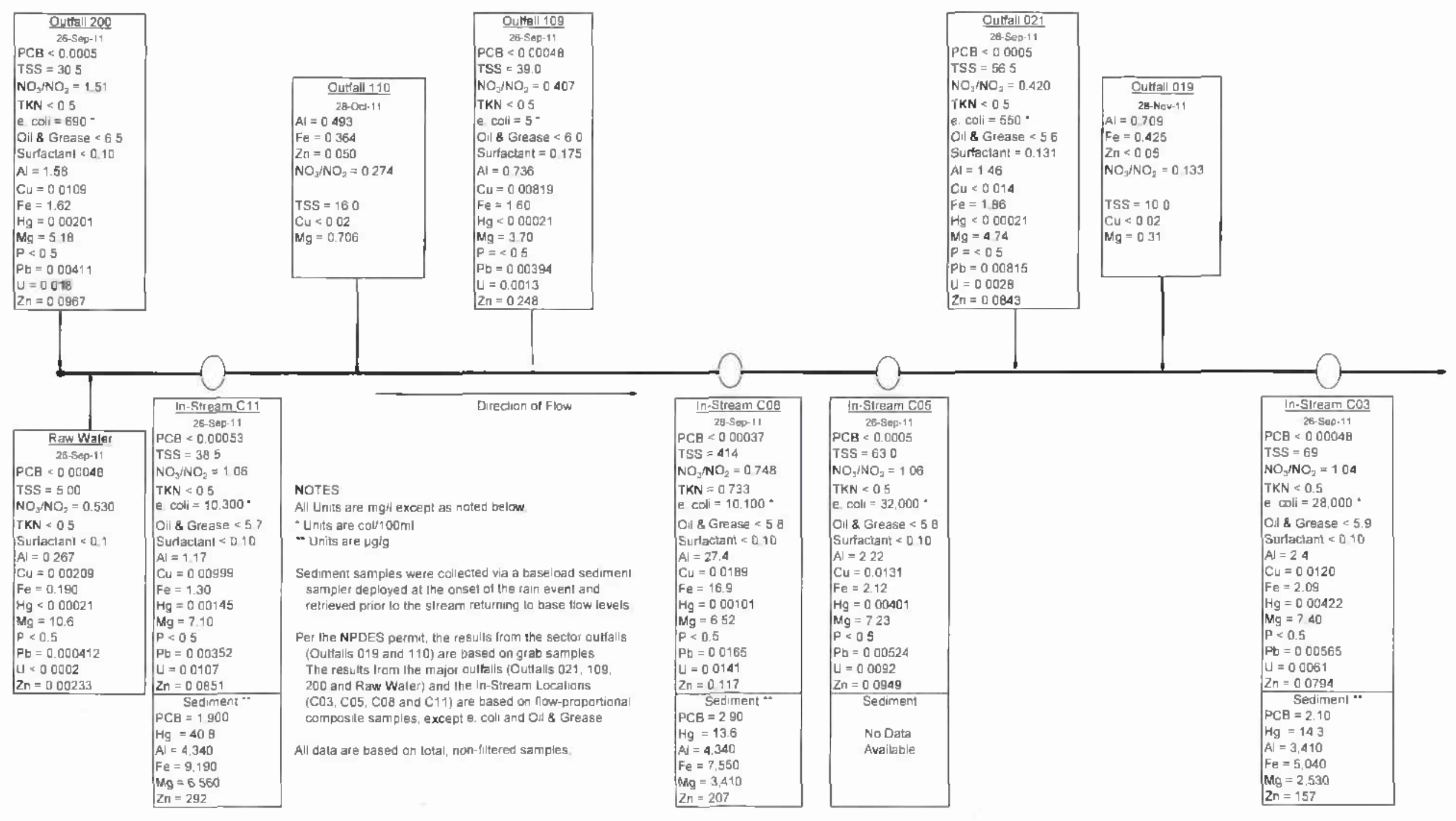




\begin{tabular}{|c|c|}
\hline 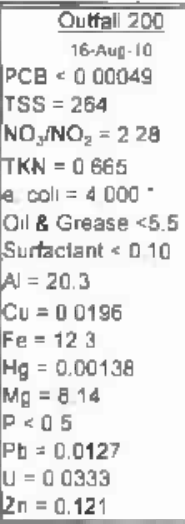 & \\
\hline & $\frac{\text { In-Sire am C11 }}{16-A \text { - } 10}$ \\
\hline 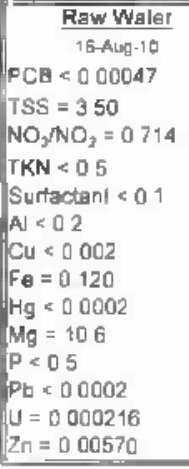 & 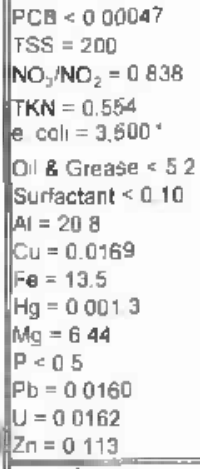 \\
\hline & 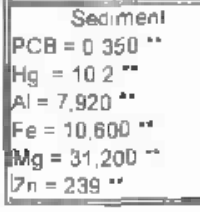 \\
\hline
\end{tabular}

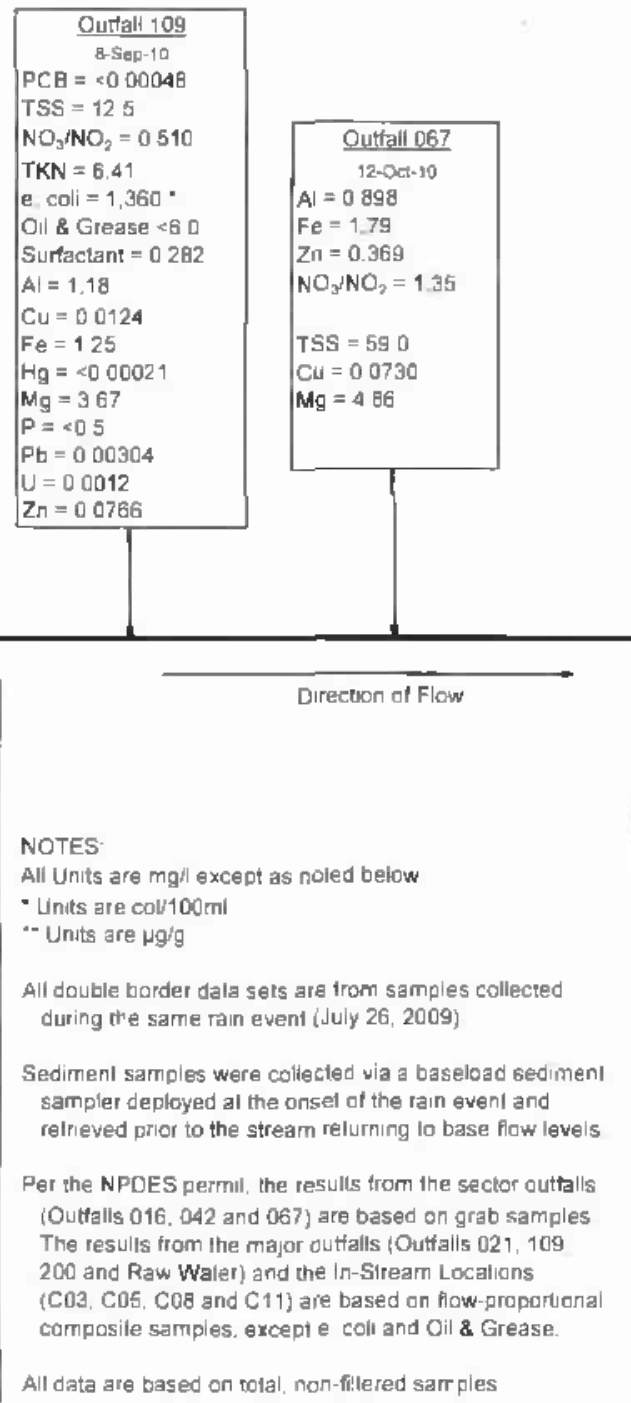

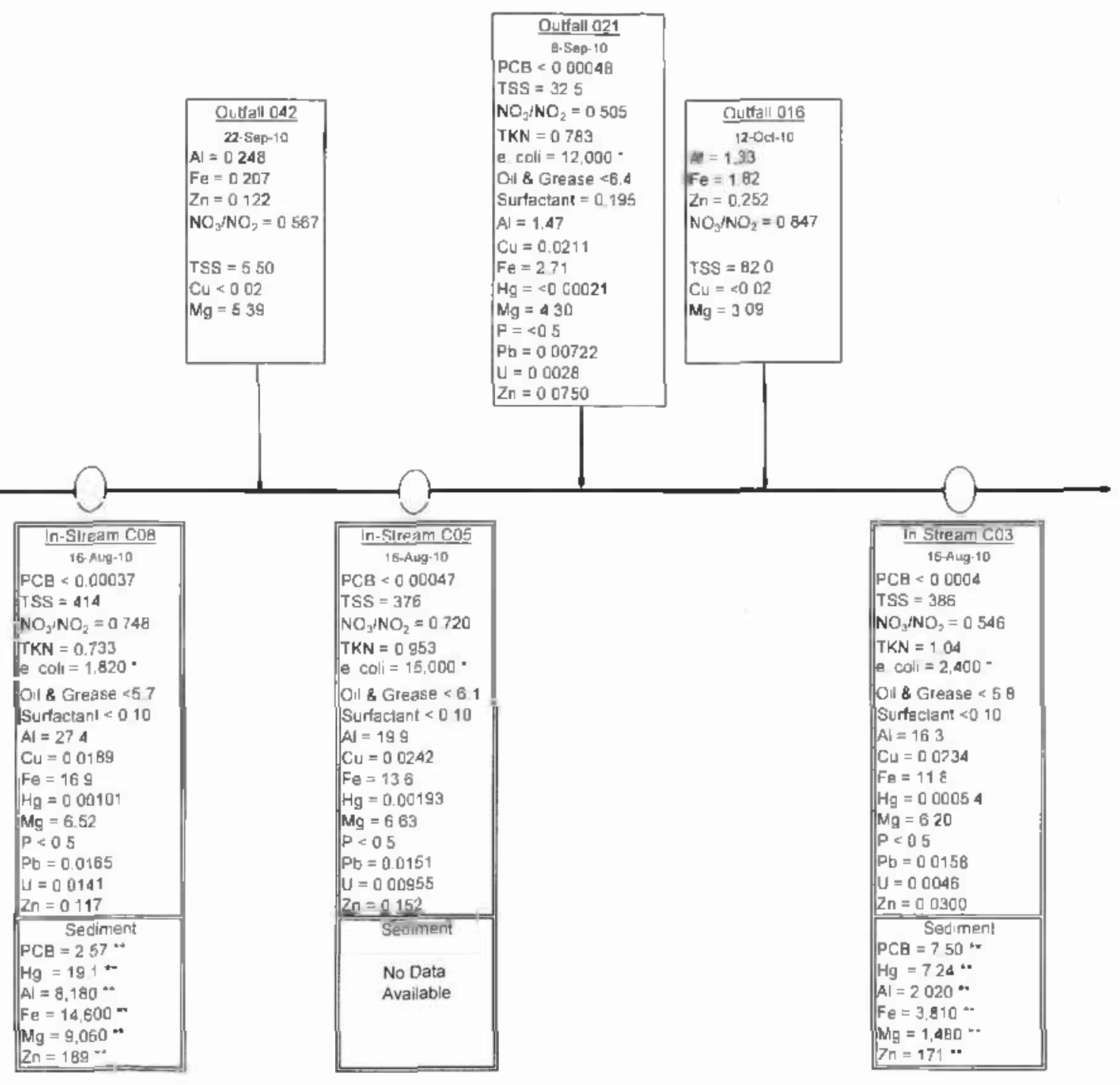




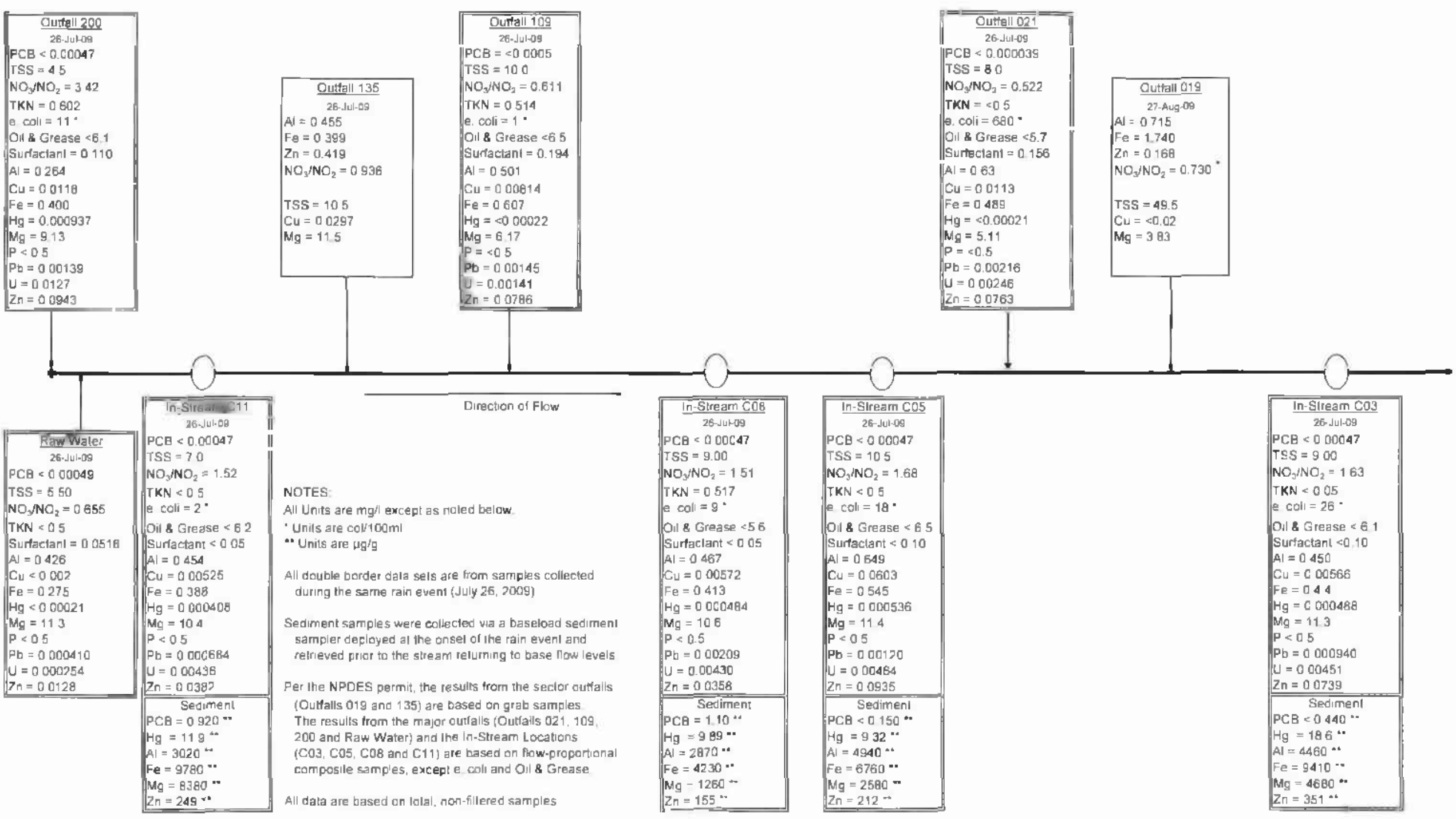




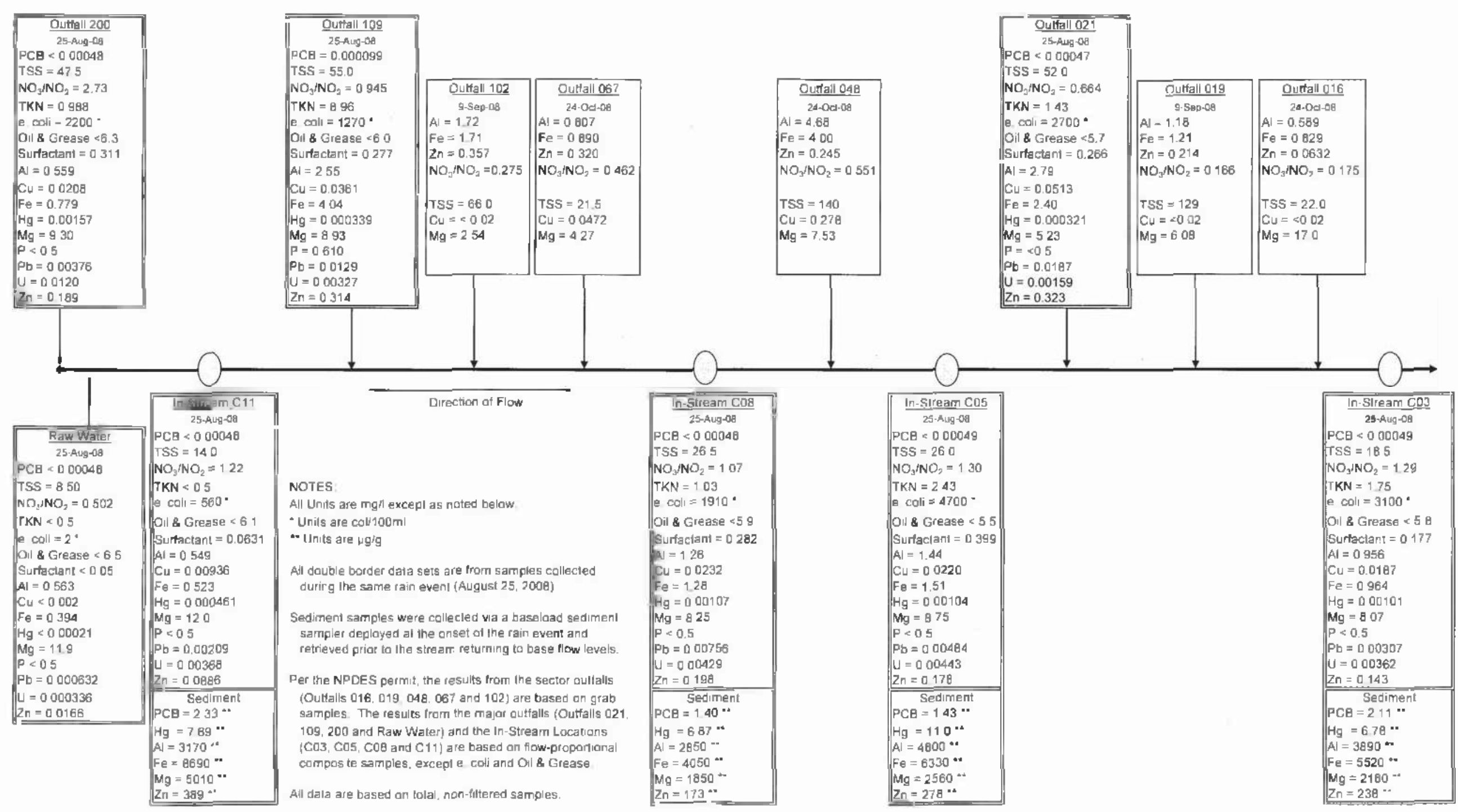


Schematic af Siorm Water Outfall Analytical Data on F.FPC

2007

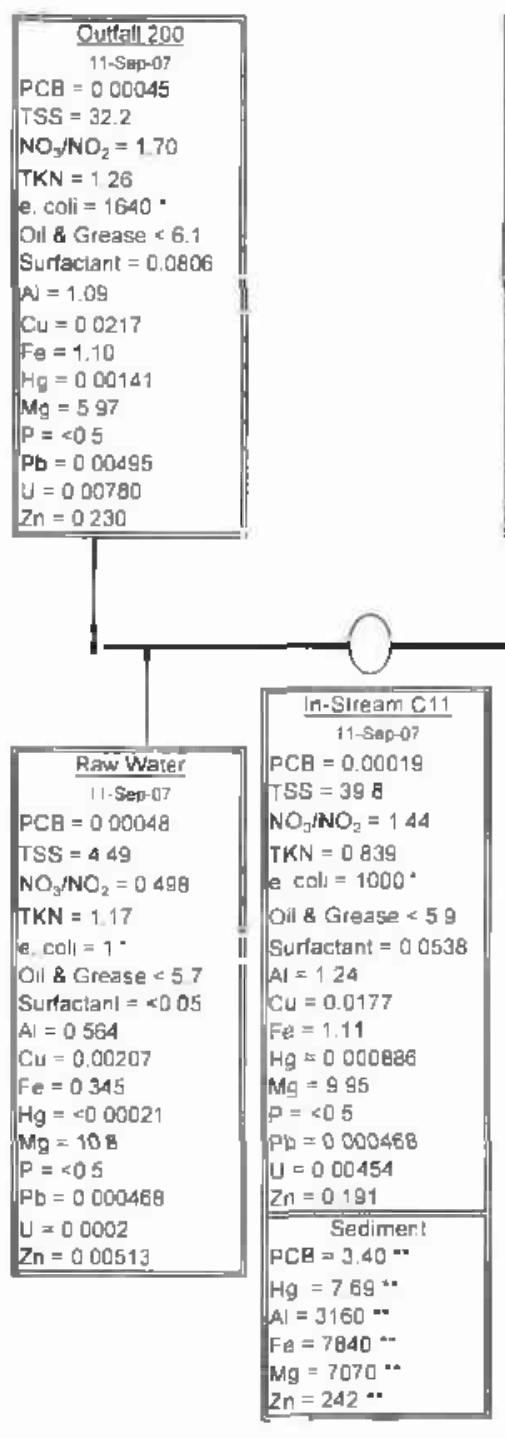

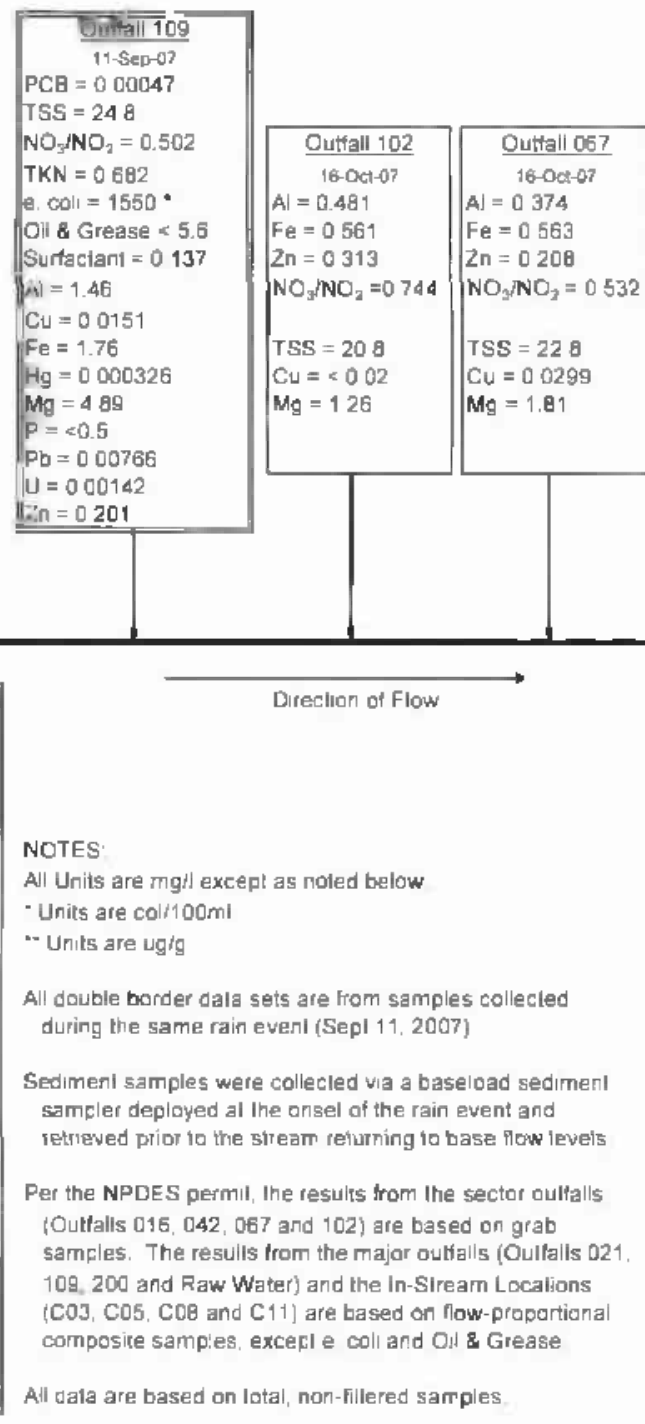

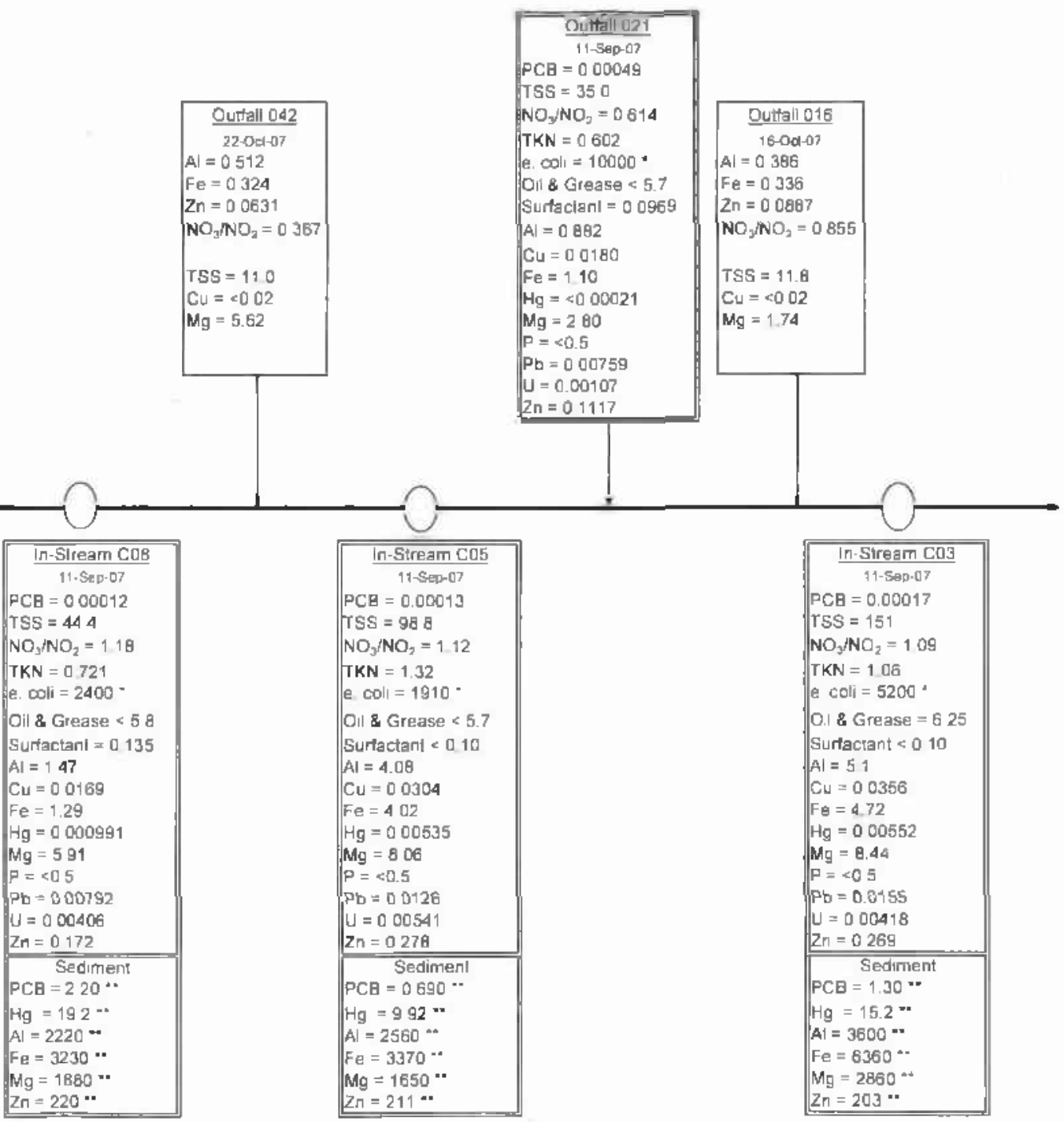


Schematic of Siorm Water Analytical Data an EFPC

2016

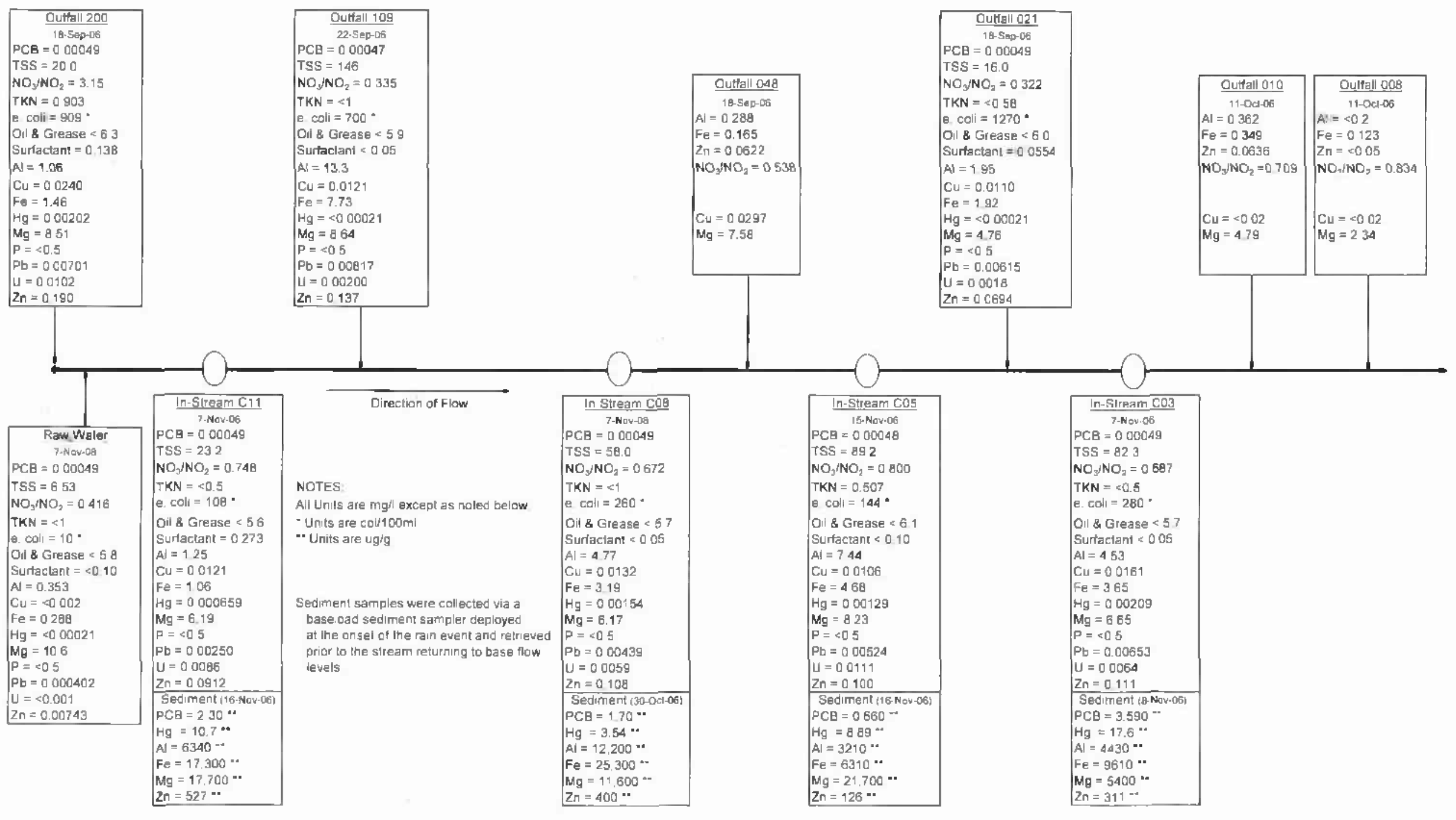


Appendix B

2013 Storm Water Analytical Results, Sector Outfalls 


\section{Sector AA \\ Outfall 014}

\begin{tabular}{|c|c|c|c|c|c|c|}
\hline Lecation & Date Collected & Sample Type & Parameter & & Result & Units \\
\hline 014 & $24-\wedge \mathrm{pr}-13$ & Ficld & $\mathrm{pH}$ & & 8.0 & Std Units \\
\hline 014 & $24-A р г-13$ & Grab & Es coli & & 120 & $\mathrm{col} / 100 \mathrm{~m}$ \\
\hline 014 & 24-Apr-13 & Grab & HexExMa: & $<$ & 5.9 & $\mathrm{mg} / \mathrm{L}$ \\
\hline 014 & 24-Aрr-13 & Grab & KjeldahIN & & 1.51 & $\mathrm{mg} / \mathrm{L}$ \\
\hline 014 & $24-A p r-13$ & Grab & $\mathrm{N}$ (CalcTot) & & 2.09 & $\mathrm{mg} / \mathrm{l}$. \\
\hline 014 & $24-A$ pr- 13 & Grab & $\mathrm{NO} 3 / \mathrm{NO} 2 \mathrm{as} \mathrm{N}$ & & 0.583 & $\mathrm{mg} / \mathrm{L}$ \\
\hline 014 & $24-\wedge p r-13$ & Grab & SuspSolids & & 55 & $\mathrm{mg} / \mathrm{L}$ \\
\hline 014 & $24-A p r-13$ & Grab & $\mathrm{Ag}$ & $<$ & 0.0002 & $\mathrm{mg} / \mathrm{L}$ \\
\hline 014 & $24-\wedge$ pr-13 & Grab & $\Lambda \mathrm{I}$ & & 0.533 & $\mathrm{mg} / \mathrm{L}$ \\
\hline 014 & $24-\wedge p r-13$ & Grab & As & $<$ & 0.002 & $\mathrm{mg} / \mathrm{L}$ \\
\hline 014 & $24-\wedge$ pr- 13 & Grab & $\mathrm{B}$ & $<$ & 0.1 & $\mathrm{mg} / \mathrm{L}$ \\
\hline 014 & $24-A p r-13$ & Grab & $\mathrm{Ba}$ & & 0.0832 & $\mathrm{mg} / \mathrm{L}$ \\
\hline 014 & 24-Apr-13 & Grab & $\mathrm{Be}$ & $<$ & 0.0002 & $\mathrm{mg} / \mathrm{L}$ \\
\hline 014 & 24-Apr- 13 & Grab & $\mathrm{Ca}$ & & 65.7 & $\mathrm{mg} / \mathrm{l}$. \\
\hline 014 & $24-$ Арг-13 & Grab & $\mathrm{Cd}$ & $<$ & 0.0002 & $\mathrm{mg} / \mathrm{L}$ \\
\hline 014 & 24-Apr-13 & Grab & Co & & 0000662 & $\mathrm{mg} / \mathrm{L}$ \\
\hline 014 & $24-А р г-13$ & Grab & $\mathrm{Cr}$ & & 0.00213 & $\mathrm{mg} / \mathrm{L}$ \\
\hline 014 & 24-Арг-13 & Grab & $\mathrm{Cu}$ & & 0.0589 & $\mathrm{mg} / \mathrm{L}$ \\
\hline 014 & 24-Арг-13 & Grab & $\mathrm{Fe}$ & & 1.75 & $\mathrm{mg} / \mathrm{L}$ \\
\hline 014 & $24-$ Арг- 13 & Grab & $\mathrm{Hg}$ & & 0.00712 & $\mathrm{mg} / \mathrm{L}$ \\
\hline 014 & $24-$ Арг-13 & Grab & $\mathrm{k}$ & & 2.48 & $\mathrm{mg} / \mathrm{l}$ \\
\hline 014 & $24-A$ pr- 13 & Grab & $\mathrm{I}, \mathrm{i}$ & $<$ & $0.0 \mathrm{I}$ & $\mathrm{mg} / \mathrm{L}$ \\
\hline 014 & $24-$ Аpr- 13 & Grab & $\mathrm{Mg}$ & & 102 & $\mathrm{mg} / \mathrm{l}$. \\
\hline 014 & 24-Apr-13 & Grab & $\mathrm{Mn}$ & & 0.265 & $\mathrm{mg} / \mathrm{L}$ \\
\hline 014 & 24-Apr-13 & Grab & Mo & $<$ & 0.0004 & $\mathrm{mg} / \mathrm{L}$ \\
\hline 014 & $24-\wedge$ pr -13 & Grab & $\mathrm{Na}$ & & 35.1 & $\mathrm{mg} / \mathrm{L}$ \\
\hline 014 & $24-\mathrm{Apr}-13$ & Grab & $\mathrm{Nb}$ & $<$ & 0.2 & $\mathrm{mg} / \mathrm{L}$ \\
\hline 014 & 24-Арг-13 & Grab & $\mathrm{Ni}$ & & 0.00308 & $\mathrm{mg} / \mathrm{L}$ \\
\hline 014 & 24-Aрr-13 & Grab & $\mathrm{P}$ & $<$ & 0.5 & $\mathrm{mg} / \mathrm{l}$ \\
\hline 014 & 24-Apr-13 & Grab & $\mathrm{Pb}$ & & 0.00247 & $\mathrm{mg} / \mathrm{l}$ \\
\hline 014 & $24-\wedge$ pr-13 & Grab & $S$ & & 9.57 & $\mathrm{mg} / \mathrm{L}$ \\
\hline 014 & 24-Apr-13 & Grab & $\mathrm{Sb}$ & $<$ & 0.001 & $\mathrm{mg} / \mathrm{L}$ \\
\hline 014 & $24-A p r-13$ & Grab & $\mathrm{Se}$ & $<$ & 0.004 & $\mathrm{mg} / \mathrm{L}$ \\
\hline 014 & $24-А$ рг-13 & Grab & Sr & & 0.171 & $\mathrm{mg} / \mathrm{I}$ \\
\hline 014 & 24-Apr-13 & Grab & Th & $<$ & 0.0002 & $\mathrm{mg} / \mathrm{L}$ \\
\hline 014 & $24-\wedge$ pr- 13 & Grab & $\mathrm{Ti}$ & $<$ & 0.05 & $\mathrm{mg} / \mathrm{L}$ \\
\hline 014 & $24-\wedge p r-13$ & Grab & $\mathrm{Tl}$ & $<$ & 0.0002 & $\mathrm{mg} / \mathrm{L}$ \\
\hline 014 & 24-Арг-13 & Grab & U & & 0.00412 & $\mathrm{mg} / \mathrm{L}$ \\
\hline 014 & 24-Арг-13 & Grab & v & $<$ & 0.02 & $\mathrm{mg} / \mathrm{L}$ \\
\hline 014 & $24-А$ pr- 13 & Grab & $\mathrm{Zn}$ & & 0.0552 & $\mathrm{mg} / \mathrm{L}$ \\
\hline 014 & 24-Apr-13 & Grab & $\mathrm{Zr}$ & $<$ & 0.2 & $\mathrm{mg} / \mathrm{L}$ \\
\hline 014 & 24-Apr-13 & Grab & Alpha & & 22 & $\mathrm{pCi} / \mathrm{L}$ \\
\hline 014 & $24-\mathrm{Apr}-13$ & Grab & Beta & & 9.5 & $\mathrm{pCi} / \mathrm{L}$ \\
\hline 014 & $24-\mathrm{Apr}-13$ & Grab & $\mathrm{U}$ & & 0.0039 & $\mathrm{mg} / \mathrm{L}$ \\
\hline 014 & 24-Арг- 13 & Grab & U-234 & $<$ & 0.005 & $w 1 \%$ \\
\hline 014 & $24-\Lambda \mathrm{pr}-13$ & Grab & U235\% & & 0.701 & wi $\%$ \\
\hline 014 & $24-\mathrm{Apr}-13$ & Grab & U. 236 & $<$ & 0.005 & wt $\%$ \\
\hline 014 & $24-A p r-13$ & Grab & U-238 & & 99.3 & w1 $\%$ \\
\hline 014 & 24-Apr-13 & Grab & $\mathrm{U}-235$ & & 0 & $\mathrm{pCi} / \mathrm{L}$ \\
\hline 014 & 24-Apr-13 & Grab & U.236 & & 0 & $\mathrm{pCi} / \mathrm{L}$ \\
\hline 014 & 24-Арг-13 & Grab & U-238 & & 0.88 & $\mathrm{pCi} / \mathrm{L}$ \\
\hline 014 & $24-A p r-13$ & Grab & U-234 & & 1.1 & $\mathrm{pCi} / \mathrm{L}$ \\
\hline
\end{tabular}




\section{Sector AA \\ Outfall 014 \\ (continued)}

\begin{tabular}{|c|c|c|c|c|c|c|}
\hline Location & Date Collected & Sample Type & Parameler & & Result & Units \\
\hline 014 & $24-$ Аpr-13 & Composite & Surfactants & & 0.278 & $\mathrm{mg} / \mathrm{L}$ \\
\hline 014 & $24-\mathrm{Apr}-13$ & Composite & SuspSolids & & 49 & $\mathrm{mg} / \mathrm{I}$. \\
\hline 014 & 24-Apr-13 & Conposite & KjeldahlN & & 1.2 & $\mathrm{mg} / \mathrm{L}$ \\
\hline 014 & $24-A p r-13$ & Composite & $\mathrm{NO} 3 / \mathrm{NO} 2 \mathrm{as} \mathrm{N}$ & & 0.826 & $\mathrm{mg} / \mathrm{L}$ \\
\hline 014 & 24-Apr-I3 & Composite & $\mathrm{N}($ CalcTot $)$ & & 2.03 & $\mathrm{mg} / \mathrm{L}$ \\
\hline $0 \mid 4$ & 24-Арг- 13 & Composite & $\mathrm{Ag}$ & $<$ & 00002 & $\mathrm{mg} / \mathrm{l}$, \\
\hline 0.4 & $24-\wedge p r-13$ & Composite & $\mathrm{Al}$ & & 0.604 & $\mathrm{mg} / \mathrm{L}$ \\
\hline 014 & 24-Apr-13 & Composite & As & $<$ & 0_002 & $\mathrm{mg} / \mathrm{L}$ \\
\hline 014 & 24-Apr-13 & Composite & $B$ & $<$ & 0.1 & $\mathrm{mg} / \mathrm{L}$ \\
\hline 014 & 24-Apr-13 & C'omposite & $\mathrm{Ba}$ & & 0.0437 & $\mathrm{mg} / \mathrm{L}$ \\
\hline 014 & 24-Арг-13 & Composite & $\mathrm{Be}$ & $<$ & 0.0002 & $\mathrm{mg} / \mathrm{L}$ \\
\hline 014 & 24-Apr-13 & Composite & $\mathrm{Ca}$ & & 32 & $\mathrm{mg} / \mathrm{L}$ \\
\hline 014 & $24-\mathrm{Apr}-13$ & Composite & $\mathrm{Cd}$ & $<$ & 0.0002 & $\mathrm{mg} / \mathrm{L}$ \\
\hline 014 & 24-Apr-13 & Composite & $\mathrm{Co}$ & & 0.000598 & $\mathrm{mg} / \mathrm{L}$ \\
\hline 014 & $24-\mathrm{Aрг-}-3$ & Composile & $\mathrm{CI}$ & & 0.00258 & $\mathrm{mg} / \mathrm{l}$. \\
\hline 014 & $24-$ Арг- 13 & Composite & $\mathrm{Cu}$ & & 0.0389 & $\mathrm{mg} / \mathrm{L}$ \\
\hline 014 & $24-\mathrm{Apr}-13$ & Composite & $\mathrm{Fe}$ & & 1.06 & $\mathrm{mg} / \mathrm{L}$ \\
\hline 014 & 24-Apr-13 & Composite & $\mathrm{I} \cdot \mathrm{gg}$ & & 0.00313 & $\mathrm{mg} / \mathrm{L}$ \\
\hline 014 & 24-Apr-13 & Composite & $\mathrm{K}$ & $<$ & 2 & $\mathrm{mg} / \mathrm{L}$ \\
\hline 014 & $24-$ Арг- 13 & Composite & $\mathrm{I}, \mathrm{i}$ & $<$ & 0.01 & $\mathrm{mg} / \mathrm{L}$ \\
\hline 014 & $24-$ Apr-13 & Composite & $\mathrm{Mg}$ & & 5.63 & $\mathrm{mg} / \mathrm{L}$ \\
\hline 014 & 24-Apr-13 & Composite & $\mathrm{Mn}$ & & 0.166 & $\mathrm{mg} / \mathrm{L}$ \\
\hline 014 & $24-\mathrm{Apr}-13$ & Composite & Mo & & $00008 / 2$ & $\mathrm{mg} / \mathrm{L}$ \\
\hline 014 & $24-\mathrm{Apr}-13$ & Composile & $\mathrm{Na}$ & & 12.6 & $\mathrm{mg} / \mathrm{l}$. \\
\hline 014 & $24-\wedge \mathrm{pr}-13$ & Composile & $\mathrm{Nb}$ & $<$ & 02 & $\mathrm{mg} / \mathrm{L}$ \\
\hline 014 & 24-Арг- 3 & Composite & $\mathrm{Ni}$ & & 0.00303 & $\mathrm{mg} / \mathrm{L}$ \\
\hline 014 & 24-Арг- 3 & Composite & $\mathrm{P}$ & $<$ & 0.5 & $\mathrm{mg} / \mathrm{L}$ \\
\hline 014 & 24-Apr-13 & Composile & $\mathrm{Pb}$ & & 0.00343 & $\mathrm{mg} / \mathrm{L}$. \\
\hline 014 & $24-A p r-13$ & Composite & $S$ & & 5.47 & $\mathrm{mg} / \mathrm{L}$ \\
\hline 014 & 24-Арг-13 & Composite & $\mathrm{Sb}$ & $<$ & 0.001 & $\mathrm{mg} / \mathrm{L}$ \\
\hline 014 & $24-\wedge p r-13$ & Composite & $\mathrm{Se}$ & $<$ & 0004 & $\mathrm{mg} / \mathrm{L}$ \\
\hline 014 & $24-$ Apr-13 & Composite & $\mathrm{Sr}$ & & 0.0794 & $\mathrm{mg} / \mathrm{L}$ \\
\hline 014 & 24-Apr-13 & Composile & Th & $<$ & 0.0002 & $\mathrm{mg} / \mathrm{I}$, \\
\hline 014 & 24-Apr-13 & Composile & $\mathrm{Ti}$ & $<$ & 0.05 & $\mathrm{mg} / \mathrm{L}$ \\
\hline 014 & $24-A p r-13$ & Composile & $\mathrm{Tl}$ & $<$ & 0.0002 & $\mathrm{mg} / \mathrm{L}$ \\
\hline 014 & $24-$ Apr- $^{-1} 3$ & Composite & $\mathrm{U}$ & & 0.00674 & $\mathrm{mg} / \mathrm{L}$ \\
\hline 014 & $24-А р г-13$ & Composite & v & $<$ & 0.02 & $\mathrm{mg} / \mathrm{L}$. \\
\hline 014 & 24-Арг-13 & Composite & $\angle n$ & & 0.0671 & $\mathrm{mg} / \mathrm{L}$ \\
\hline 014 & $24-A p r-13$ & Composite & $\mathrm{Zr}$ & $<$ & 0.2 & $\mathrm{mg} / \mathrm{L}$ \\
\hline
\end{tabular}




\section{Sector AA \\ Outfall 014 \\ (continued)}

\begin{tabular}{|c|c|c|c|c|c|c|}
\hline Location & Date Collected & Sample Type & Parameter & & Result & Units \\
\hline 014 & $24-A p r-13$ & Composite & Alpha & & 5.7 & $\mathrm{pCi} / \mathrm{L}$ \\
\hline 014 & $24-A p r-13$ & Composite & Be1a & & 1.7 & $\mathrm{pCi} / \mathrm{L}$ \\
\hline 014 & 24-Apr-13 & Composite & U & & 0.007 & $\mathrm{mg} / \mathrm{L}$ \\
\hline 014 & $24-A p r-13$ & Composite & U. -234 & & 0.81 & $\mathrm{pCi} / \mathrm{L}$ \\
\hline 014 & 24-Apr-13 & Composite & $\mathrm{U}-235$ & & 0.011 & $\mathrm{pCi} / \mathrm{L}$ \\
\hline 014 & $24-A p r-13$ & Composite & U-236 & & 0.018 & $\mathrm{pCi} / \mathrm{L}$ \\
\hline 014 & 24-Apr-13 & Composite & $\mathrm{U}-238$ & & 2.1 & $\mathrm{pCi} / \mathrm{L}$ \\
\hline 014 & 24-Apr-13 & Conposite & $\mathrm{U}-234$ & & 0.0283 & w1 $\%$ \\
\hline 014 & $24-\wedge$ рг-13 & Composite & U235\% & & 0514 & $w \%$ \\
\hline 014 & $24-A p r-13$ & Compositc & $\mathrm{U}-236$ & & 0.0157 & wt $\%$ \\
\hline 014 & 24-Apr-13 & Composite & $\mathrm{U}-238$ & & 99.4 & wt $\%$ \\
\hline 014 & 24-Арг-13 & Composite & РCB & J & 0.04 & $\mathrm{ug} / \mathrm{L}$ \\
\hline 014 & $24-А р г-13$ & Composite & PCB-1016 & $1 !$ & 0.5 & $\lg / 1$ \\
\hline 014 & $24-A p r-13$ & Composite & РCB-1221 & $\mathrm{U}$ & 0.5 & $\mathrm{ug} / 1$. \\
\hline 014 & 24-Apr-13 & Composite & PCB-1232 & $\mathrm{U}$ & 0.5 & ug/L \\
\hline 014 & 24-Apr-13 & Composite & PC'B-1242 & $\mathrm{U}$ & 0.5 & ug $/ \mathrm{L}$ \\
\hline 014 & $24-A p r-13$ & Composite & РCB-1248 & U! & 0.5 & $\mathrm{ug} / \mathrm{L}$ \\
\hline 014 & $24-A p r-13$ & Compositc & РCB-1254 & $\mathrm{U}$ & 05 & $\mathrm{ug} / \mathrm{L}$ \\
\hline 014 & $24-A p r-13$ & Composite & PCB- 1260 & .1 & 0.04 & $\mathrm{ug} / \mathrm{L}$ \\
\hline $0 \mid 4$ & $24-А р г-13$ & Composite & РCB-1262 & U) & 0.5 & $\mathrm{ug} / \mathrm{L}$. \\
\hline 014 & $24-\mathrm{Apr}-13$ & Composite & РCB- 1268 & $\mathrm{U}$ & 0.5 & $\| \mathrm{g} / \mathrm{L}$ \\
\hline
\end{tabular}




\section{Sector AA \\ Outfall 067}

\begin{tabular}{|c|c|c|c|c|c|c|}
\hline Location & Date Collected & Sample Typc & Parameter & & Result & Units \\
\hline 067 & 4-Арг-13 & Field & $\mathrm{pH}$ & & 80 & Std Units \\
\hline 067 & 4-Apr-13 & Grab & Es. coli & & 9 & $\mathrm{col} / 100 \mathrm{ml}$ \\
\hline 067 & 4-Apr-13 & Grab & HexExMatl & $<$ & 5.7 & $\mathrm{mg} / \mathrm{L}$ \\
\hline 067 & 4-Арг-13 & Grab & SuspSolids & & 8.5 & $\mathbf{m g} / \mathrm{L}$ \\
\hline 067 & 4-Apr-13 & Grab & KjeldahIN & $<$ & 0.5 & $\mathrm{mg} / \mathrm{L}$ \\
\hline 067 & $4-\wedge p r-13$ & Grab & $\mathrm{NO} 3 / \mathrm{NO} 2 \mathrm{asN}_{\mathrm{N}}$ & & 067 & $\mathrm{mg} / \mathrm{L}$ \\
\hline 067 & 4-Арг-13 & Grab & $N($ Calc Tot $)$ & & 0.67 & $\mathrm{mg} / \mathrm{L}$ \\
\hline 067 & 4-Aрr-13 & Grab & $\mathrm{Ag}$ & $<$ & 0.0002 & $\mathrm{mg} / \mathrm{L}$ \\
\hline 067 & $4-А р г-13$ & Grab & Al & $<$ & 0.2 & $\mathrm{mg} / \mathrm{L}$ \\
\hline 067 & 4-Арг-13 & Grab & As & $<$ & 0.002 & $\mathrm{mg} / \mathrm{L}$ \\
\hline 067 & 4-Aрr-13 & Grab & $B$ & $<$ & 0.1 & $\mathrm{mg} / \mathrm{l}$ \\
\hline 067 & $4-\wedge p r-13$ & Grab & $\mathrm{Ba}$ & & 0.0877 & $\mathrm{mg} / \mathrm{L}$ \\
\hline 067 & 4-Арг-13 & Grab & $\mathrm{Bc}$ & $<$ & 0.0002 & $\mathrm{mg} / \mathrm{L}$ \\
\hline 067 & 4-Apr-13 & Grab & $\mathrm{Ca}$ & & 59.9 & $\mathrm{mg} / \mathrm{L}$ \\
\hline 067 & 4-Apr-13 & Grab & Cd & $<$ & 0.0002 & $\mathrm{mg} / \mathrm{L}$ \\
\hline 067 & 4-Арг-13 & Grab & $\mathrm{Co}$ & & 0.000354 & $\mathrm{mg} / \mathrm{L}$ \\
\hline 067 & 4-Apr-13 & Grab & $\mathrm{Cr}$ & & 0.0031 & $\mathrm{mg} / \mathrm{L}$ \\
\hline 067 & 4-Арг-13 & Grab & $\mathrm{Cu}$ & & 0.015 & $\mathrm{mg} / \mathrm{L}$ \\
\hline 067 & 4-Арг-I3 & Grab & $\mathrm{Fe}$ & & 0.209 & $\mathrm{mg} / \mathrm{L}$ \\
\hline 067 & 4-Аpr-13 & Grab & $\mathrm{Hg}$ & $<$ & 0.053 & $\mathrm{ug} / \mathrm{L}$ \\
\hline 067 & 4-Арг-13 & Grab & $\mathrm{k}$ & & 2.89 & $\mathrm{mg} / \mathrm{L}$ \\
\hline 067 & 4-Apr-13 & Grab & $\mathrm{Li}$ & $<$ & 0.01 & $\mathrm{mg} / \mathrm{L}$ \\
\hline 067 & 4-Apr-13 & Grab & $\mathrm{Mg}$ & & 9.08 & $\mathrm{mg} / \mathrm{L}$ \\
\hline 067 & $4-A p r-13$ & Grab & $\mathrm{Mn}$ & & 0.0146 & $\mathrm{mg} / \mathrm{L}$. \\
\hline 067 & 4-Арг-13 & Grab & Mo & & 0.000684 & $\mathrm{mg} / \mathrm{L}$ \\
\hline 067 & 4-Арг-13 & Grab & $\mathrm{Na}$ & & 50.3 & $\mathrm{mg} / \mathrm{L}$ \\
\hline 067 & 4-Apr-13 & Grab & $\mathrm{Nb}$ & $<$ & 0.2 & $\mathrm{mg} / \mathrm{L}$ \\
\hline 067 & 4-Apr-13 & Grab & $\mathrm{Ni}$ & $<$ & 0.002 & $\mathrm{mg} / \mathrm{L}$ \\
\hline 067 & $4-\wedge p r-13$ & Grab & $P$ & $<$ & 0.5 & $\mathrm{mg} / \mathrm{l}$, \\
\hline 067 & $4-A p r-13$ & Grab & $\mathrm{Pb}$ & & 0.00237 & $\mathrm{mg} / \mathrm{L}$ \\
\hline 067 & 4-Apr-13 & Grab & $S$ & & 9.54 & $\mathrm{mg} / \mathrm{L}$ \\
\hline 067 & $4-\Lambda p r-13$ & Grab & $\mathrm{Sb}$ & & 0.00133 & $\mathrm{mg} / \mathrm{L}$ \\
\hline 067 & 4-Арг-13 & Girab & $\mathrm{Se}$ & $<$ & 0004 & $\mathrm{mg} / \mathrm{L}$ \\
\hline 067 & 4-Apr-13 & Grab & $\mathrm{Sr}$ & & 0.161 & $\mathrm{mg} / \mathrm{L}$ \\
\hline 067 & $4-\wedge$ рг-J3 & Grab & $\left.\mathrm{T}\right|_{1}$ & $<$ & 0.0002 & $\mathrm{mg} / \mathrm{L}$ \\
\hline 067 & 4-Apr-13 & Grab & $\mathrm{Ti}$ & $<$ & 0.05 & $\mathrm{mg} / \mathrm{L}$ \\
\hline 067 & 4-Apr-13 & Grab & $\mathrm{Tl}$ & $<$ & 0.0002 & $\mathrm{mg} / \mathrm{L}$ \\
\hline 067 & 4-Apr-13 & Grab & v & $<$ & 0.02 & $\mathrm{mg} / \mathrm{L}$ \\
\hline 067 & 4-Арг-13 & Grab & $\mathrm{Zn}$ & & 0.0592 & $\mathrm{mg} / \mathrm{L}$ \\
\hline 067 & 4-Apr-13 & Grab & $7 r$ & $<$ & 0.2 & $\mathrm{mg} / \mathrm{L}$ \\
\hline 067 & 4-Apr-13 & Grab & U & & 0.0019 & $\mathrm{mg} / \mathrm{L}$ \\
\hline 067 & $4-A p r-13$ & Grab & Alpha & & 1.7 & $\mathrm{pCi} / \mathrm{L}$ \\
\hline 067 & 4-Aрr-13 & Grab & Beta & & 6 & $\mathrm{pCi} / \mathrm{L}$ \\
\hline 067 & 4-Apr-13 & Grab & $\mathrm{U}-234$ & $<$ & 0.005 & wt \% \\
\hline 067 & 4-Apr-I3 & Grab & U $235 \%$ & & 11.4 & $w t \%$ \\
\hline 067 & $4-\wedge p r-13$ & Grab & U-236 & $<$ & 0.005 & wt $\%$ \\
\hline 067 & 4-Apr-13 & Grab & U-238 & & 88.6 & wt $\%$ \\
\hline 067 & 4-Apr-13 & Grab & U-235 & & 0.074 & $\mathrm{pCi} / \mathrm{L}$ \\
\hline 067 & 4-Apr-13 & Grab & U-236 & & 0.029 & $\mathrm{pCi} / \mathrm{L}$ \\
\hline 067 & 4-Apr-13 & Grab & U. 238 & & 0.38 & $\mathrm{pCi} / \mathrm{L}$ \\
\hline 067 & 4-Apr-13 & Grab & U-234 & & 0.85 & $\mathrm{pCi} / \mathrm{L}$ \\
\hline
\end{tabular}




\section{Sector AA \\ Outfall 067 \\ (continued)}

\begin{tabular}{|c|c|c|c|c|c|c|}
\hline Location & Dale Collected & Sample Type & Parameter & & Result & Units \\
\hline 067 & 4-Apr-13 & Composite & SuspSolids & & 46.5 & $\mathrm{mg} / \mathrm{L}$ \\
\hline 067 & $4-A p r-\mid 3$ & Composite & Surfactants & & 0.129 & $\mathrm{mg} / \mathrm{L}$ \\
\hline 067 & 4-Арг-13 & Composite & Kjeldah|N & $<$ & 0.5 & $\mathrm{mg} / \mathrm{l}$. \\
\hline 067 & 4-Арг-13 & Composite & $\mathrm{NO} 3 / \mathrm{NO} 2 \mathrm{asN}$ & & 0.185 & $\mathrm{mg} / \mathrm{l}$. \\
\hline 067 & 4-Арr-13 & Composite & N(CalcTot) & & 0.185 & $\mathrm{mg} / \mathrm{l}$. \\
\hline 067 & 4-Apr-13 & Composite & $\mathrm{Ag}$ & $<$ & 0.0002 & $\mathrm{mg} / \mathrm{L}$ \\
\hline 067 & 4-Арг-13 & Composite & $\mathrm{Al}$ & & 1.04 & $\mathrm{mg} / \mathrm{L}$ \\
\hline 067 & 4-Арг-13 & Composite & $\Lambda \mathrm{s}$ & $<$ & 0.002 & $\mathrm{mg} / \mathrm{l}$. \\
\hline 067 & 4-Apr-13 & Composite & B & $<$ & 0.1 & $\mathrm{mg} / \mathrm{L}$ \\
\hline 067 & $4-A p r-13$ & Composite & $\mathrm{Ba}$ & & 0.0268 & $\mathrm{mg} / \mathrm{L}$ \\
\hline 067 & 4-Apr-13 & Composite & Be & $<$ & 0.0002 & $\mathrm{mg} / \mathrm{L}$. \\
\hline 067 & 4-Apr-13 & Composite & $\mathrm{Ca}$ & & 18.5 & $\mathrm{mg} / \mathrm{L}$. \\
\hline 067 & 4-Apr-13 & Composite & $\mathrm{Cd}$ & $<$ & 0.0002 & $\mathrm{mg} / \mathrm{L}$ \\
\hline 067 & 4-Арг-13 & Composite & $\mathrm{Co}$ & & 0.000516 & $\mathrm{mg} / \mathrm{L}$ \\
\hline 067 & 4-Apr-13 & Composite & $\mathrm{Cr}$ & & 0.00507 & $\mathrm{mg} / \mathrm{L}$ \\
\hline 067 & 4-Арг-13 & Composite & Cu & & 0.0157 & $\mathrm{mg} / \mathrm{L}$ \\
\hline 067 & 4-Apr-13 & Composite & $\mathrm{Fe}$ & & 1.06 & $\mathrm{mg} / \mathrm{L}$ \\
\hline 067 & 4-Арг-13 & Composite & $\mathrm{Hg}$ & $<$ & 0.053 & $u g / L$ \\
\hline 067 & 4-Apr-13 & Composite & $k$ & $<$ & 2 & $\mathrm{mg} / \mathrm{L}$. \\
\hline 067 & 4-Арг-13 & Composite & $\mathrm{Li}$ & $<$ & 0.01 & $\mathrm{mg} / \mathrm{l}$. \\
\hline 067 & 4-Арг-।3 & Composite & $\mathrm{Mg}$ & & 3.26 & $\mathrm{mg} / \mathrm{L}$ \\
\hline 067 & 4-Арг-13 & Composite & $\mathrm{Mn}$ & & 0.0703 & $\mathrm{mg} / \mathrm{L}$. \\
\hline 067 & 4-Apr-13 & Composite & Mo & & 0.000464 & $\mathrm{mg} / \mathrm{L}$ \\
\hline 067 & 4-Арг-।3 & Composite & $\mathrm{Na}$ & & 6.81 & $\mathrm{mg} / \mathrm{L}$ \\
\hline 067 & $4-\wedge p r-13$ & Composite & $\mathrm{Nb}$ & $<$ & 0.2 & $\mathrm{mg} / \mathrm{L}$ \\
\hline 067 & 4-Арг-13 & Composite & $\mathrm{N}_{i}$ & $<$ & 0.002 & $\mathrm{mg} / \mathrm{h}$. \\
\hline 067 & 4-Арг-13 & Composite & $\mathrm{P}$ & $<$ & 0.5 & $\mathrm{mg} / \mathrm{L}$ \\
\hline 067 & 4-Apr-13 & Composite & $\mathrm{Pb}$ & & 0.00569 & $\mathrm{mg} / \mathrm{L}$ \\
\hline 067 & 4-Арг-13 & Composite & $\mathrm{s}$ & & 1.82 & $\mathrm{mg} / \mathrm{L}$ \\
\hline 067 & 4-Арг-13 & Composite & $\mathrm{Sb}$ & $<$ & 0.001 & $\mathrm{mg} / \mathrm{L}$ \\
\hline 067 & 4-Арг-13 & Composite & $\mathrm{Se}$ & $<$ & 0.004 & $\mathrm{mg} / \mathrm{L}$ \\
\hline 067 & 4-Арг-13 & Composite & $\mathrm{Sr}$ & & $0.035]$ & $\mathrm{mg} / \mathrm{L}$ \\
\hline 067 & $4-\wedge$ pr-13 & Composite & Th & $<$ & 0.0002 & $\mathrm{mg} / \mathrm{L}$ \\
\hline 067 & $4-\wedge p r-13$ & Composite & $\mathrm{Ti}$ & $<$ & 0.05 & $\mathrm{mg} / \mathrm{L}$ \\
\hline 067 & 4-Арг-13 & Composite & $\mathrm{TI}$ & $<$ & 0.0002 & $\mathrm{mg} / \mathrm{L}$ \\
\hline 067 & 4-Apr-13 & Composite & v & $<$ & 002 & $\mathrm{mg} / \mathrm{L}$ \\
\hline 067 & 4-Apr-13 & Composite & $\mathrm{Zn}$ & & 0.128 & $\mathrm{mg} / \mathrm{L}$ \\
\hline 067 & 4-Apr-13 & Composite & $\mathrm{Zr}_{\mathrm{I}}$ & $<$ & 0.2 & $\mathrm{mg} / \mathrm{L}$ \\
\hline
\end{tabular}




\section{Sector AA}

Outfall 067

(continued)

\begin{tabular}{|c|c|c|c|c|c|c|}
\hline Location & Date Collected & Sample Type & Parameter & & Result & Units \\
\hline 067 & 4-Apr-13 & Composite & $\mathrm{U}$ & & 0.000492 & $\mathrm{mg} / \mathrm{L}$ \\
\hline 067 & 4-Apr-13 & Composite & Alpha & & 7.6 & $\mathrm{pCi} / \mathrm{l}$. \\
\hline 067 & 4-Арт-13 & Composite & Beta & & 10 & $\mathrm{pCi} / \mathrm{L}$ \\
\hline 067 & 4-Apr-13 & Composite & U. -234 & & $N / A$ & $w t \%$ \\
\hline 067 & $4-\wedge$ рг-13 & Composite & U235\% & & $\mathrm{N} / \mathrm{A}$ & $w 1 \%$ \\
\hline 067 & 4-Apr-13 & Composite & U -236 & & $\mathrm{~N} / \mathrm{A}$ & wt $\%$ \\
\hline 067 & 4-Apr-13 & Composite & U -238 & & $\mathrm{~N} / \mathrm{A}$ & wit $\%$ \\
\hline 067 & 4-Apr-13 & Composite & U- -234 & & 0.34 & $\mathrm{pCi} / \mathrm{L}$ \\
\hline 067 & 4-Apr-I3 & Composite & U -235 & & -00068 & $\mathrm{pCi} / \mathrm{l}$. \\
\hline 067 & 4-Арr-13 & Compositc & UJ-236 & & -0.025 & $\mathrm{pCi} / \mathrm{L}$ \\
\hline 067 & 4-Apr- $\mid 3$ & Composite & U -238 & & 0.2 & $\mathrm{pCi} / \mathrm{L}$ \\
\hline 067 & 4-Apr-13 & Composite & PCB & J & 0.045 & $\mathrm{ug} / \mathrm{L}$ \\
\hline 067 & $4-\wedge \mathrm{pr}-13$ & Composite & PCB-1016 & $\mathrm{U}$ & 0.46 & $\mathrm{ug} / \mathrm{L}$ \\
\hline 067 & 4-Apr-13 & Composite & PCB-1221 & $\mathrm{U}$ & 0.46 & $\mathrm{ug} / \mathrm{L}$ \\
\hline 067 & $4-\wedge p r-13$ & Composite & PCB-1232 & $\mathrm{U}$ & 0.46 & $\mathrm{ug} / \mathrm{L}$ \\
\hline 067 & $4-\wedge \mathrm{pr}-13$ & Composite & PCB-1242 & $\mathrm{U}$ & 0.46 & $\mathrm{ug} / \mathrm{L}$ \\
\hline 067 & 4-Apr-13 & Composite & PCB- 1248 & U & 0.46 & $\mathrm{ug} / \mathrm{L}$ \\
\hline 067 & 4-Apr-13 & Composite & PCB- 1254 & $\mathrm{U}$ & 0.46 & $\mathrm{ug} / \mathrm{L}$ \\
\hline 067 & 4-Арг-13 & Composite & $\mathrm{PCB} \cdot 1260$ & $\mathrm{~J}$ & 0.045 & $\mathrm{ug} / \mathrm{L}$ \\
\hline 067 & 4-Apr-13 & Composite & PC13-1262 & U & 0.46 & $\mathrm{ug} / \mathrm{L}$ \\
\hline 067 & 4-Apr-13 & Composite & PCB-1268 & $\mathrm{U}$ & 0.46 & $\mathrm{ug} / \mathrm{L}$ \\
\hline
\end{tabular}




\section{Sector AA}

Outfall 102

\begin{tabular}{|c|c|c|c|c|c|c|}
\hline Location & Date Collected & Sample Type & Parameter & & Resul1 & Units \\
\hline 102 & 4-Aрг- 13 & Field & $\mathrm{pH}$ & & 7.9 & Sid Units \\
\hline 102 & 4-Apr-13 & Grab & Es coli & & 35 & $\mathrm{col} / 100 \mathrm{ml}$ \\
\hline 102 & 4-Арг-13 & Grab & HexExMatl & $<$ & 5.7 & $\mathrm{mg} / \mathrm{l}$ \\
\hline 102 & 4-Apr-I3 & Grab & SuspSolids & & 15 & $\mathrm{mg} / \mathrm{L}$ \\
\hline 102 & 4-Apr-13 & Cirab & KjeldalılN & $<$ & 0.5 & $\mathrm{mg} / \mathrm{L}$ \\
\hline 102 & $4-\Lambda p r-13$ & Grab & NO3/NO2asN & & 0.351 & $\mathrm{mg} / \mathrm{l}$ \\
\hline 102 & 4-Apr-I3 & Girab & N(CalcTot) & & $0.35\rfloor$ & $\mathrm{mg} / \mathrm{L}$ \\
\hline 102 & 4-Apr-13 & Grab & $\mathrm{Ag}$ & $<$ & 00002 & $\mathrm{mg} / \mathrm{l}$ \\
\hline 102 & 4-Арг- 3 & Grab & $\mathrm{Al}$ & $<$ & 0.2 & $\mathrm{mg} / \mathrm{L}$ \\
\hline 102 & 4-Apr-13 & Grab & As & $<$ & 0.002 & $\mathrm{mg} / \mathrm{l}$. \\
\hline 102 & $4-A p r-13$ & Grab & B & $<$ & 0.1 & $\mathrm{mg} / \mathrm{L}$ \\
\hline 102 & $4-A p r-13$ & Grab & $\mathrm{Ba}$ & & 0.0141 & $\mathrm{mg} / \mathrm{L}$ \\
\hline 102 & 4-Apr-13 & Grab & $\mathrm{Be}$ & $<$ & 0.0002 & $\mathrm{mg} / \mathrm{I}$ \\
\hline 102 & 4-Apr-13 & Grab & $\mathrm{Ca}$ & & 9.67 & $\mathrm{mg} / \mathrm{L}$ \\
\hline 102 & 4-Apr- 13 & Grab & $C \mathrm{Cd}$ & $<$ & 0.0002 & $\mathrm{mg} / \mathrm{L}$ \\
\hline 102 & $4-\wedge p r-13$ & Grab & $\mathrm{Co}$ & & 0.000248 & $\mathrm{mg} / \mathrm{L}$ \\
\hline 102 & 4-Apr-13 & Grab & $\mathrm{Cr}$ & $<$ & 0.002 & $\mathrm{mg} / \mathrm{L}$ \\
\hline 102 & 4-Apr-13 & Grab & $\mathrm{Cu}$ & & 0.0116 & $\mathrm{mg} / \mathrm{h}$ \\
\hline 02 & 4-Арг-13 & Grab & $\mathrm{Fe}$ & & 0.17 & $\mathrm{mg} / \mathrm{L}$ \\
\hline 102 & 4-Apr-13 & Grab & $\mathrm{Hg}$ & $<$ & 0.053 & $u g / L$ \\
\hline 102 & 4-Арг-13 & Grab & $\mathrm{k}$ & $<$ & 2 & $\mathrm{mg} / \mathrm{L}$ \\
\hline 102 & 4-Арг-13 & Grab & $\mathrm{Li}$ & $<$ & 0.01 & $\mathrm{mg} / \mathrm{L}$ \\
\hline 102 & 4-Apr-13 & Girab & $\mathrm{Mg}$ & & 1.12 & $\mathrm{mg} / \mathrm{L}$ \\
\hline 102 & 4-Apr-13 & Grab & $\mathrm{Mn}$ & & 0.0102 & $\mathrm{mg} / \mathrm{L}$ \\
\hline 102 & $4-А р г-13$ & Grab & Mo & $<$ & 0.0004 & $\mathrm{mg} / \mathrm{L}$ \\
\hline 102 & $4-\wedge \mathrm{pr}-13$ & Grab & $\mathrm{Na}$ & & 1.23 & $\mathrm{mg} / \mathrm{L}$ \\
\hline 102 & 4-Apr- 13 & Grab & $\mathrm{Nb}$ & $<$ & 0.2 & $\mathrm{mg} / \mathrm{L}$ \\
\hline 102 & 4-Apr-13 & Grab & $\mathrm{Ni}$ & $<$ & 0.002 & $\mathrm{mg} / \mathrm{L}$ \\
\hline 102 & 4-Арr-13 & Girab & $\mathbf{p}$ & $<$ & 0.5 & $\mathrm{mg} / \mathrm{L}$ \\
\hline 102 & $4-А р г-13$ & Grab & $\mathrm{Pb}$ & & 0.00317 & $\mathrm{mg} / \mathrm{l}$. \\
\hline 102 & 4.Apr- 13 & Grab & $s$ & & 136 & $\mathrm{mg} / \mathrm{L}$ \\
\hline 102 & 4-Apr-13 & Grab & $\mathrm{Sb}$ & $<$ & 0.001 & $\mathrm{mg} / \mathrm{L}$ \\
\hline 102 & 4-Apr-13 & Grab & $\mathrm{Se}$ & $<$ & 0.004 & $\mathrm{mg} / \mathrm{L}$ \\
\hline 102 & 4-Apr-13 & Grab & $\mathrm{Sr}$ & & 0.0163 & $\mathrm{mg} / \mathrm{L}$ \\
\hline 102 & 4-Apr-13 & Grab & Th & $<$ & 0.0002 & $\mathrm{mg} / \mathrm{L}$ \\
\hline 102 & 4-Apr-13 & Grab & $\mathrm{Ti}$ & $<$ & 0.05 & $\mathrm{mg} / \mathrm{L}$ \\
\hline 102 & 4-Apr-13 & Grab & $\mathrm{Tl}$ & $<$ & 0.0002 & $\mathrm{mg} / \mathrm{L}$ \\
\hline 102 & 4-Apr-13 & Grab & $\mathrm{u}$ & . & 0.00327 & $\mathrm{mg} / \mathrm{L}$ \\
\hline 102 & 4-Apr-13 & Grab & $\mathrm{V}$ & $<$ & 0.02 & $\mathrm{mg} / \mathrm{L}$ \\
\hline 102 & 4-Aрг-13 & Grab & $\mathrm{Zn}$ & & 0.0986 & $\mathrm{mg} / \mathrm{L}$ \\
\hline 102 & 4-Apr-13 & Grab & $\mathrm{Zr}$ & $<$ & 0.2 & $\mathrm{mg} / \mathrm{L}$ \\
\hline 102 & 4-Apr-13 & Grab & Alpha & & 2.4 & $\mathrm{pC} \mathrm{j} / \mathrm{L}$ \\
\hline 102 & 4-Арг-13 & Grab & Beta & & 11 & $\mathrm{pC} / \mathrm{i} / \mathrm{L}$ \\
\hline 102 & 4-Apr-13 & Grab & $\mathrm{U}$ & & 0.0031 & $\mathrm{mg} / \mathrm{L}$ \\
\hline 102 & 4-Арг-13 & Grab & $\mathrm{U}-234$ & $<$ & 0.005 & wt $\%$ \\
\hline 102 & 4-Apr- 13 & Grab & $\mathrm{U} 235 \%$ & & 0.622 & wt $\%$ \\
\hline 102 & 4-Apr-13 & Grab & $\mathrm{U}-236$ & $<$ & 0.005 & w1 \% \\
\hline 102 & 4-Apr-13 & Grab & $\mathrm{U}-238$ & & 99.4 & wt $\%$ \\
\hline 102 & 4-Apr-13 & Grab & $\mathrm{U}-235$ & & 0.018 & $\mathrm{pCi} / \mathrm{L}$ \\
\hline 102 & 4-Apr-13 & Grab & U-236 & & 0.014 & $\mathrm{pCi} / \mathrm{L}$ \\
\hline 102 & 4-Aрr-13 & Grab & U-238 & & 1.1 & $\mathrm{pCi} / \mathrm{L}$ \\
\hline 102 & 4-Арг-13 & Grab & U-234 & & 0.57 & $\mathrm{pCi} / \mathrm{L}$. \\
\hline
\end{tabular}




\section{Sector AA}

Outfall 102

(continued)

\begin{tabular}{|c|c|c|c|c|c|c|}
\hline Location & Date Collected & Sample Type & Parameter & & Result & Units \\
\hline 102 & 4-Apr-13 & Composite & SuspSolids & & 7 & $\mathrm{mg} / \mathrm{L}$ \\
\hline 102 & 4-Apr-13 & Composite & Surfactants & $<$ & 0.1 & $\mathrm{mg} / \mathrm{L}$ \\
\hline 102 & 4-Apr-13 & Composite & KjeldalıIN & $<$ & 0.5 & $\mathrm{mg} / \mathrm{L}$ \\
\hline 102 & 4-Apr- 13 & Composite & $\mathrm{NO} 3 / \mathrm{NO} 2 \mathrm{asN}$ & & 0.163 & $\mathrm{mg} / \mathrm{L}$ \\
\hline 102 & 4-Apr-13 & Composite & $\mathrm{N}(\mathrm{CalcTo})$ & & 0.163 & $\mathrm{mg} / \mathrm{L}$ \\
\hline 102 & 4-Apr-13 & Composite & $\mathrm{Ag}$ & $<$ & 0.0002 & $\mathrm{mg} / \mathrm{L}$ \\
\hline 102 & $4-\wedge p r-13$ & Composite & $\wedge 1$ & & 0.206 & $\mathrm{mg} / \mathrm{L}$ \\
\hline 102 & 4-Apr-13 & Composite & As & $<$ & 0.002 & $\mathrm{mg} / \mathrm{L}$ \\
\hline 102 & 4-Apr-13 & Composite & B & $<$ & 01 & $\mathrm{mg} / \mathrm{L}$ \\
\hline 102 & 4-Apr-13 & Composite & Ba & & 0.011 & $\mathbf{m g} / \mathrm{l}$. \\
\hline 102 & 4-Apr-13 & Composite & $\mathrm{Be}$ & $<$ & 0.0002 & $\mathrm{mg} / \mathrm{L}$ \\
\hline 102 & $4-\wedge p r-13$ & Composite & $\mathrm{Ca}$ & & 8.04 & $\mathrm{mg} / \mathrm{I}$ \\
\hline 102 & 4-Apr-13 & Composite & $\mathrm{Cd}$ & $<$ & 0.0002 & $\mathrm{mg} / \mathrm{L}$ \\
\hline 102 & 4-Apr-13 & Composite & $\mathrm{Co}$ & $<$ & 0.0002 & $\mathrm{mg} / \mathrm{L}$ \\
\hline 102 & 4-Apr- 13 & Composite & $\mathrm{Cr}$ & $<$ & 0.002 & $\mathrm{mg} / \mathrm{L}$ \\
\hline 102 & 4-Apr- 13 & Composile & $\mathrm{Cu}$ & & 0.00802 & $\mathrm{mg} / \mathrm{L}$ \\
\hline 102 & 4-Apr-13 & Composite & $\mathrm{Fe}$ & & 0.209 & $\mathrm{mg} / \mathrm{L}$ \\
\hline 102 & 4-Apr-13 & Composite & $\mathrm{Hlg}$ & $<$ & 0.053 & $\mathrm{ug} / \mathrm{L}$ \\
\hline 102 & 4-Apr-13 & Composite & $k$ & $<$ & 2 & $\mathrm{mg} / \mathrm{L}$ \\
\hline 102 & $4-\wedge p r-13$ & Composite & I.i & $<$ & 0.01 & $\mathrm{mg} / \mathrm{L}$ \\
\hline 102 & 4-Apr-13 & Composite & $\mathrm{Mg}$ & & 0.743 & $\mathrm{mg} / \mathrm{L}$ \\
\hline 102 & 4-Apr- 13 & Composite & $\mathrm{Mn}$ & & 0.00777 & $\mathrm{mg} / \mathrm{L}$ \\
\hline 102 & 4-Apr-13 & Composite & Mo & $<$ & 0.0004 & $\mathrm{mg} / \mathrm{L}$ \\
\hline 102 & 4-Apr-13 & Composite & $\mathrm{Na}$ & & 1.35 & $\mathrm{mg} / \mathrm{L}$ \\
\hline 102 & $4-\wedge p r-13$ & Composite & $\mathrm{Nb}$ & $<$ & 0.2 & $\mathrm{mg} / \mathrm{L}$ \\
\hline 102 & $4-\wedge \mathrm{pr}-13$ & Composite & $\mathrm{Ni}$ & $<$ & 0002 & $\mathrm{mg} / \mathrm{l}$. \\
\hline 102 & 4-Apr-13 & Composite & $P$ & $<$ & 0.5 & $\mathrm{mg} / \mathrm{L}$ \\
\hline 102 & $4-\wedge p r-13$ & Composite & $\mathrm{Pb}$ & & 0.00261 & $\mathrm{mg} / \mathrm{L}$. \\
\hline 102 & 4-Apr-13 & Composite & s & & 0.809 & $\mathrm{mg} / \mathrm{L}$. \\
\hline 102 & 4-Арг-13 & Composite & Sb & $<$ & 0.001 & $\mathrm{mg} / \mathrm{I}$, \\
\hline 102 & 4-Apr-13 & Composite & $\mathrm{Se}$ & $<$ & 0.004 & $\mathrm{mg} / \mathrm{L}$ \\
\hline 102 & 4-Apr-13 & Composite & $\mathrm{Sr}$ & & 0.0131 & $\mathrm{mg} / \mathrm{L}$ \\
\hline 102 & $4-\mathrm{Apr}-13$ & Composite & Th & $<$ & 0.0002 & $\mathrm{mg} / \mathrm{L}$ \\
\hline 102 & 4-Apr-13 & Composite & $\mathrm{Ti}$ & $<$ & 0.05 & $\mathrm{mg} / \mathrm{L}$ \\
\hline 102 & 4-Apr-13 & Composite & $\mathrm{Tl}$ & $<$ & 0.0002 & $\mathrm{mg} / \mathrm{L}$ \\
\hline 102 & $4-\wedge p r-13$ & Composite & $\mathrm{U}$ & & 0.00118 & $\mathrm{mg} / \mathrm{L}$ \\
\hline 102 & 4-Apr- 13 & Composite & $\mathrm{v}$ & $<$ & 0.02 & $\mathrm{mg} / \mathrm{L}$. \\
\hline 102 & $4-A p r-13$ & Composite & $\mathrm{Zn}_{n}$ & & 0.0862 & $\mathrm{mg} / \mathrm{L}$ \\
\hline 102 & 4-Apr-13 & Composite & 7r & $<$ & 0.2 & $\mathrm{mg} / \mathrm{L}$ \\
\hline
\end{tabular}




\section{Sector AA \\ Outfall 102 \\ (continued)}

\begin{tabular}{|c|c|c|c|c|c|c|}
\hline Location & Date Collected & Sample Type & Parameter & & Result & Units \\
\hline 102 & 4-Apr-13 & Composite & U & & 0.0011 & $\mathrm{mg} / \mathrm{L}$ \\
\hline 102 & 4-Apr-13 & Composite & Alpha & & 2.8 & $\mathrm{pCi} / \mathrm{L}$ \\
\hline 102 & 4-Арг-। 3 & Composite & Beta & & 0.97 & $\mathrm{pCi} / \mathrm{L}$ \\
\hline 102 & 4-Aрг-13 & Composite & $\mathrm{U}-234$ & & 0.112 & wt $\%$ \\
\hline 102 & 4.Apr-13 & Compositc & $\mathrm{U} 235 \%$ & & 1.27 & wt $\%$ \\
\hline 102 & $4-А р r-\mid 3$ & Composite & U- 236 & & 0.353 & $w 1 \%$ \\
\hline 102 & 4-Apr-13 & Composite & U]-238 & & 98.3 & $w t \%$ \\
\hline 102 & $4-A p I-13$ & Composite & U -235 & & -0.018 & pCi/l. \\
\hline 102 & 4-Apr-13 & Conposite & U. 236 & & 0.014 & $\mathrm{pCi} / \mathrm{L}$ \\
\hline 102 & 4-Apr-13 & Composite & U-238 & & 0.28 & $\mathrm{pCi} / \mathrm{L}$ \\
\hline 102 & 4-Apr-13 & Composite & $\mathrm{U}-234$ & & 0.26 & $\mathrm{pC}_{\mathrm{i}} / \mathrm{L}$ \\
\hline 102 & 4-Apr-13 & Composite & PCB & $\mathrm{U}$ & 0.46 & $\mathrm{ug} / \mathrm{L}$ \\
\hline 102 & 4.Apr-13 & Composite & PCB-1016 & U & 0.46 & ug/L \\
\hline 102 & 4-Apr-13 & Composite & PCB-122! & [] & 0.46 & $u \mathrm{~g} / \mathrm{L}$ \\
\hline 102 & 4-Apr-13 & Composite & PCB- 1232 & $\mathrm{U}$ & 0.46 & บg/L \\
\hline 102 & 4-Aрг-13 & Composite & PCB- 1242 & U & 0.46 & ॥! $/ \mathrm{L}$ \\
\hline 102 & 4-Apr-13 & Composite & РСВ- 1248 & $\mathrm{U}$ & 0.46 & ug/L \\
\hline 102 & 4-Apr-13 & Composite & PCB-1254 & $\mathrm{U}$ & 0.46 & $\mathrm{ug} / \mathrm{L}$ \\
\hline 102 & $4-A p r-13$ & Composite & PCB- 1260 & $\mathrm{U}$ & 0.46 & $\mathrm{ug} / \mathrm{L}$ \\
\hline 102 & $4-A p r-13$ & Composite & PCB- 1262 & $\mathrm{U}$ & 0.46 & $\mathrm{ug} / \mathrm{g}$. \\
\hline 102 & 4-Apr-13 & Composite & PCB- 1268 & $\mathrm{U}$ & 0.46 & $\mathrm{ug} / \mathrm{L}$ \\
\hline
\end{tabular}




\section{Sector K \\ Outfall S06}

\begin{tabular}{|c|c|c|c|c|c|c|}
\hline Location & Date Collected & Sample Type & Parameter & & Result & llnits \\
\hline S06 & 24-Apr-13 & Field & $\mathrm{pH}$ & & 7.4 & Std Units \\
\hline S06 & 24-Арг-13 & Grab & COD & & 5.00 & $\mathrm{mg} / \mathrm{L}$ \\
\hline S06 & 24-Apr-13 & Grab & $\mathrm{BOD}$ & $<$ & 5.0 & $\mathrm{mg} / \mathrm{l}$ \\
\hline S06 & $24-A p r-13$ & Grab & HexExMatl & $<$ & 59 & $\mathrm{mg} / \mathrm{L}$ \\
\hline S06 & $24-\mathrm{Apr}-13$ & Grab & Cyanide & $<$ & 0.05 & $\mathrm{mg} / \mathrm{L}$ \\
\hline S06 & 24-Apr-13 & Grab & SuspSolids & & 7.50 & $\mathrm{mg} / \mathrm{L}$ \\
\hline $\mathrm{S} 06$ & $24-\mathrm{Apr}-13$ & Grab & KjeldahıN & $<$ & 0.5 & $\mathrm{mg} / \mathrm{L}$ \\
\hline S06 & 24-Apr-13 & Grab & NH3asN & $<$ & 0.2 & $\mathrm{mg} / \mathrm{L}$ \\
\hline S06 & $24-A p r-13$ & Grab & $\mathrm{N}($ CalcTot $)$ & & 27.2 & $\mathrm{mg} / \mathrm{L}$ \\
\hline S06 & 24-Apr-13 & Grab & $\mathrm{NO} 3 / \mathrm{NO} 2 \mathrm{asN}$ & & 27.2 & $\mathrm{mg} / \mathrm{L}$ \\
\hline S06 & 24-Арг-13 & Grab & $\mathrm{Ag}$ & $<$ & 0.0002 & $\mathrm{mg} / \mathrm{L}$ \\
\hline S06 & $24-A p r-13$ & Grab & A] & & 0.579 & $\mathrm{mg} / \mathrm{L}$ \\
\hline S06 & $24-\wedge \mathrm{pr}-13$ & Grab & As & $<$ & 0.002 & $\mathrm{mg} / \mathrm{L}$ \\
\hline S06 & $24-A p r-13$ & Grab & $\mathrm{B}$ & & 0.292 & $\mathrm{mg} / \mathrm{L}$ \\
\hline S06 & $24-\wedge \mathrm{pr}-13$ & Grab & $\mathrm{Ba}$ & & 0.154 & $\mathrm{mg} / \mathrm{L}$ \\
\hline S06 & 24-Apr-13 & Grab & $\mathrm{Be}$ & $<$ & 0.0002 & $\mathrm{mg} / \mathrm{L}$ \\
\hline S06 & $24-A p r-13$ & Grab & $\mathrm{Ca}$ & & 105 & $\mathrm{mg} / \mathrm{L}$ \\
\hline S06 & $24-A p r-13$ & Grab & $\mathrm{Cd}$ & & 0.00188 & $\mathrm{mg} / \mathrm{L}$ \\
\hline S06 & $24-A p r-13$ & Grab & $\mathrm{Co}$ & & 0.00107 & $\mathrm{mg} / \mathrm{L}$ \\
\hline S06 & $24-A p r-13$ & Grab & $\mathrm{Cr}$ & $<$ & 0.002 & $\mathrm{mg} / \mathrm{L}$ \\
\hline S06 & $24-\wedge \mathrm{pr}-13$ & Grab & $\mathrm{Cu}$ & $<$ & 0.002 & $\mathrm{mg} / \mathrm{L}$ \\
\hline S06 & $24-A p r-13$ & Grab & $\mathrm{Fe}$ & & 0.537 & $\mathrm{mg} / \mathrm{L}$ \\
\hline S06 & 24-Aрг-I3 & Grab & $\mathrm{Hg}$ & $<$ & 0.053 & $\mathrm{ug} / \mathrm{L}$ \\
\hline S06 & $24-\wedge \mathrm{pr}-13$ & Grab & $\mathrm{K}$ & & 2.67 & $\mathrm{mg} / \mathrm{L}$ \\
\hline S06 & 24-Apr-13 & Grab & $\mathrm{Li}$ & & 0.0733 & $\mathrm{mg} / \mathrm{L}$ \\
\hline S06 & 24-Apr-I3 & Grab & $\mathrm{Mg}$ & & 14.8 & $\mathrm{mg} / \mathrm{l}$. \\
\hline S06 & $24-A p r-13$ & Grab & $\mathrm{Mn}$ & & 0.538 & $\mathrm{mg} / \mathrm{L}$ \\
\hline S06 & $24-A p r-13$ & Grab & Mo & $<$ & 0.0004 & $\mathrm{mg} / \mathrm{L}$ \\
\hline S06 & 24-Apr-13 & Grab & $\mathrm{Na}$ & & 22.2 & $\mathrm{mg} / \mathrm{L}$ \\
\hline S06 & $24-\AA \mathrm{pr}-13$ & Grab & $\mathrm{Nb}$ & $<$ & 0.2 & $\mathrm{mg} / \mathrm{L}$ \\
\hline S06 & 24-Apr-13 & Grab & $\mathrm{Ni}$ & & 0.00966 & $\mathrm{mg} / \mathrm{L}$ \\
\hline S06 & 24-Apr-I3 & Grab & $\mathrm{P}$ & $<$ & 0.5 & $\mathrm{mg} / \mathrm{L}$ \\
\hline S06 & $24-A p r-13$ & Grab & $\mathrm{Pb}$ & $<$ & 0.001 & $\mathrm{mg} / \mathrm{L}$ \\
\hline S06 & $24-A p r-13$ & Grab & $S$ & & 8.46 & $\mathrm{mg} / \mathrm{l}$ \\
\hline S06 & $24-A p r-13$ & Grab & $\mathrm{Sb}$ & $<$ & 0.001 & $\mathrm{mg} / \mathrm{L}$ \\
\hline S06 & $24-\mathrm{Apr}-13$ & Grab & $\mathrm{Se}$ & $<$ & 0.004 & $\mathrm{mg} / \mathrm{L}$ \\
\hline S06 & $24-\mathrm{Apr}-13$ & Grab & $\mathrm{Sr}$ & & 0.303 & $\mathrm{mg} / \mathrm{L}$ \\
\hline S06 & $24-А p r-13$ & Grab & Th & $<$ & 0.0002 & $\mathrm{mg} / \mathrm{L}$ \\
\hline S06 & $24-\mathrm{Apr}-13$ & Grah & $\mathrm{Ti}$ & $<$ & 0.05 & $\mathrm{mg} / \mathrm{L}$ \\
\hline S06 & $24-A p r-13$ & Grab & $\mathrm{Tl}$ & $<$ & 0.0002 & $\mathrm{mg} / \mathrm{L}$ \\
\hline S06 & $24-A p r-13$ & Grab & U & & $0.096 \mathrm{l}$ & $\mathrm{mg} / \mathrm{L}$ \\
\hline S06 & $24-A p r-13$ & Grab & v & $<$ & 0.02 & $\mathrm{mg} / \mathrm{L}$ \\
\hline S06 & 24-Apr-13 & Grab & $\mathrm{Zn}$ & & 0.00558 & $\mathrm{mg} / \mathrm{L}$ \\
\hline S06 & 24-Apr-13 & Grab & $\mathrm{Zr}$ & $<$ & 0.2 & $\mathrm{mg} / \mathrm{L}$ \\
\hline
\end{tabular}




\section{Sector K \\ Outfall S06 \\ (continued)}

\begin{tabular}{|c|c|c|c|c|c|c|}
\hline Location & Datc Collected & Sample Type & Parameter & & Result & Units \\
\hline S06 & $24-A p r-13$ & Composile & $\mathrm{COD}$ & & 11.0 & $\mathrm{mg} / \mathrm{L}$ \\
\hline S06 & $24-\mathrm{Apr}-13$ & Composite & BOD & $<$ & 50 & $\mathrm{mg} / \mathrm{L}$ \\
\hline S06 & 24-Apr-13 & Composite & SuspSolids & & 13.5 & $\mathrm{mg} / \mathrm{L}$ \\
\hline S06 & $24-$ Арг-13 & Composite & KjeldahIN & $<$ & 05 & $\mathrm{mg} / \mathrm{L}$ \\
\hline S06 & $24-A p r-13$ & Composite & NH3asN & $<$ & 0.2 & $\mathrm{mg} / \mathrm{L}$ \\
\hline S06 & $24-\mathrm{Apr}-13$ & Composite & N(CalcTot) & & 2688 & $\mathrm{mg} / \mathrm{L}$ \\
\hline $\mathrm{S} 06$ & 24-Apr-13 & Composite & $\mathrm{NO} 3 / \mathrm{NO} 2 \mathrm{as} \mathrm{N}$ & & 26.8 & $\mathrm{mg} / \mathrm{L}$ \\
\hline S06 & $24-\Lambda \mathrm{pr}-13$ & Composite & Cyanide & $<$ & 0.05 & $\mathrm{mg} / \mathrm{l}$, \\
\hline S06 & $24-A p r-13$ & Composite & $\mathrm{Ag}$ & $<$ & 0.0002 & $\mathrm{mg} / \mathrm{L}$ \\
\hline S06 & $24-A p r-I 3$ & Composite & $\mathrm{Al}$ & & 0.927 & $\mathrm{mg} / \mathrm{L}$ \\
\hline S06 & $24-A p r-13$ & Composite & As & $<$ & 0.002 & $\mathrm{mg} / \mathrm{l}$. \\
\hline S06 & $24-A$ pr- 13 & Composile & B & & 0.277 & $\mathrm{mg} / \mathrm{L}$ \\
\hline S06 & $24-A p r-13$ & Composite & $\mathrm{Ba}$ & & 0.156 & $\mathrm{mg} / \mathrm{L}$ \\
\hline $\mathrm{S} 06$ & 24-Apr-13 & Composite & $\mathrm{Be}$ & $<$ & 0.0002 & $\mathrm{mg} / \mathrm{L}$ \\
\hline S06 & $24-$ Apr- 13 & Composite & $\mathrm{Ca}$ & & 994 & $\mathrm{mg} / \mathrm{L}$ \\
\hline S06 & 24-Apr-13 & Composite & $\mathrm{Cd}$ & & 0.00321 & $\mathrm{mg} / \mathrm{L}$ \\
\hline S06 & 24-Арг-13 & Composite & $\mathrm{Co}$ & & 0.00137 & $\mathrm{mg} / \mathrm{L}$ \\
\hline S06 & $24-\wedge \mathrm{pr}-13$ & Composite & $\mathrm{Cr}$ & $<$ & 0.002 & $\mathrm{mg} / \mathrm{l}$ \\
\hline S06 & $24-\mathrm{Apr}-13$ & Compositc & $\mathrm{Cu}$ & $<$ & 0.002 & $\mathrm{mg} / \mathrm{L}$ \\
\hline S06 & $24-A p r-13$ & Composite & $\mathrm{Fe}$ & & 0.691 & $\mathrm{mg} / \mathrm{L}$ \\
\hline S06 & $24-\wedge \mathrm{pr}-13$ & Composite & $\mathrm{llg}$ & $<$ & 0.053 & $\mathrm{ug} / \mathrm{l}$. \\
\hline S06 & 24-Apr- 13 & Composite & $\mathrm{K}$ & & 2.96 & $\mathrm{mg} / \mathrm{L}$ \\
\hline S06 & $24-\wedge \mathrm{pr}-13$ & Composite & $\mathrm{Li}$ & & 0.0689 & $\mathrm{mg} / \mathrm{l}$, \\
\hline$\$ 06$ & $24-A p r-13$ & Compositc & $\mathrm{Mg}$ & & 14.5 & $\mathrm{mg} / \mathrm{L}$ \\
\hline S06 & $24-\mathrm{Apr}-13$ & Composite & Mn & & 0.661 & $\mathrm{mg} / \mathrm{L}$ \\
\hline S06 & $24-\mathrm{Apr}-13$ & Composite & Mo & $<$ & 0.0004 & $\mathrm{mg} / \mathrm{L}$ \\
\hline$\$ 06$ & $24-\mathrm{Apr}-13$ & Composite & $\mathrm{Na}$ & & 21.5 & $\mathrm{mg} / \mathrm{L}$ \\
\hline S06 & $24-\Lambda \mathrm{pr}-13$ & Composite & $\mathrm{Nb}$ & $<$ & 0.2 & $\mathrm{mg} / \mathrm{t}$. \\
\hline S06 & $24-\mathrm{Apr}-13$ & Composite & $\mathrm{Ni}$ & & 0.0119 & $\mathrm{mg} / \mathrm{L}$ \\
\hline S06 & $24-A$ pr- 13 & Composite & $\mathrm{P}$ & $<$ & 0.5 & $\mathrm{mg} / \mathrm{L}$. \\
\hline S06 & $24-A p r-13$ & Composite & $\mathrm{Pb}$ & $<$ & $0.00 \mathrm{I}$ & $\mathrm{mg} / \mathrm{L}$ \\
\hline S06 & 24-Apr-13 & Composite & $\mathrm{s}$ & & 8.70 & $\mathrm{mg} / \mathrm{L}$ \\
\hline S06 & $24-\mathrm{Apr}-13$ & Composite & $\mathrm{Sb}$ & $<$ & $0.00 \mathrm{I}$ & $\mathrm{mg} / \mathrm{L}$ \\
\hline S06 & $24-A$ pr- 13 & Composite & $\mathrm{Se}$ & $<$ & 0.004 & $\mathrm{mg} / \mathrm{L}$ \\
\hline S06 & $24-А р г-13$ & Composite & $\mathrm{Sr}$ & & 0.304 & $\mathrm{mg} / \mathrm{L}$ \\
\hline S06 & $24-\mathrm{Apr}-13$ & Composite & Th & $<$ & 0.0002 & $\mathrm{mg} / \mathrm{L}$ \\
\hline S06 & $24-\mathrm{Apr}-13$ & Composite & $\mathrm{Ti}$ & $<$ & 0.05 & $\mathrm{mg} / \mathrm{L}$ \\
\hline S06 & $24-A p^{-}-13$ & Composite & $\mathrm{TI}$ & $<$ & 0.0002 & $\mathrm{mg} / \mathrm{L}$ \\
\hline S06 & $24-\mathrm{Apr}-13$ & Composite & $\mathrm{U}$ & & 0.121 & $\mathrm{mg} / \mathrm{L}$ \\
\hline S06 & $24-\mathrm{Apr}-13$ & Composite & v & $<$ & 0.02 & $\mathrm{mg} / \mathrm{L}$ \\
\hline S06 & 24-Apr-13 & Composite & $\mathrm{Zn}$ & & 0.00871 & $\mathrm{mg} / \mathrm{L}$ \\
\hline S06 & $24-A p r-13$ & Composite & $\mathrm{Zr}$ & $<$ & 0.2 & $\mathrm{mg} / \mathrm{L}$ \\
\hline
\end{tabular}


Sector L

Outfall S17

\begin{tabular}{|c|c|c|c|c|c|c|}
\hline Location & Date Collected & Sample Type & Parameter & & Result & Units \\
\hline $\mathrm{S} 17$ & $4-\wedge p r-13$ & Field & $\mathrm{pH}$ & & 7.7 & Std Units \\
\hline S17 & 4-Apr-13 & Grab & $\mathrm{N}(\mathrm{Ca}$ eTol $)$ & & 1.61 & $\mathrm{mg} / \mathrm{L}$ \\
\hline$\$ 17$ & $4-\wedge p r-13$ & Grab & $\mathrm{NO} 3 / \mathrm{NO} 2 \mathrm{asN}$ & & $1.6]$ & $\mathrm{mg} / \mathrm{l}$. \\
\hline$\$ 17$ & 4-Арг-13 & Grab & SuspSolids & & 3.50 & $\mathrm{mg} / \mathrm{L}$ \\
\hline S17 & 4-Apr-13 & Grab & kjeldahıN & $<$ & 0.5 & $\mathrm{mg} / \mathrm{L}$ \\
\hline Si7 & 4-Apr-13 & Grab & NH3asN & $<$ & 0.2 & $\mathrm{mg} / \mathrm{L}$ \\
\hline S17 & 4-Apr-13 & Grab & $\mathrm{Ag}$ & $<$ & 0.0002 & $\mathrm{mg} / \mathrm{L}$ \\
\hline$\$ 17$ & 4-Apr-13 & Grab & Al & $<$ & 0.2 & $\mathrm{mg} / \mathrm{L}$ \\
\hline S17 & $4-\Lambda p r-13$ & Grab & As & $<$ & 0.002 & $\mathrm{mg} / \mathrm{L}$ \\
\hline S17 & 4-Apr-13 & Grab & B & $<$ & 0.1 & $\mathrm{mg} / \mathrm{l}$ \\
\hline S17 & 4-Apr-13 & Grab & $\mathrm{Ba}$ & & 0.0509 & $\mathrm{mg} / \mathrm{L}$ \\
\hline S17 & 4-Apr-13 & Grab & $\mathrm{Be}$ & $<$ & 0.0002 & $\mathrm{mg} / \mathrm{L}$ \\
\hline S17 & 4-Apr-13 & Grab & $\mathrm{Ca}$ & & 35.6 & $\mathrm{mg} / \mathrm{L}$ \\
\hline $\mathrm{S} 17$ & 4-Apr-13 & Grab & $\mathrm{Cd}$ & $<$ & 0.0002 & $\mathrm{mg} / \mathrm{l}$. \\
\hline S17 & 4-Apr-13 & Grab & Co & $<$ & 0.0002 & $\mathrm{mg} / \mathrm{L}$ \\
\hline$\$ 17$ & 4-Apr-13 & Grab & $\mathrm{Cr}$ & $<$ & 0.002 & $\mathrm{mg} / \mathrm{L}$ \\
\hline S17 & 4-Apr-13 & Grab & $\mathrm{Cu}$ & $<$ & 0.002 & $\mathrm{mg} / \mathrm{L}$ \\
\hline S17 & 4-Apr-13 & Grab & $\mathrm{Fe}$ & & 0.103 & $\mathrm{mg} / \mathrm{L}$ \\
\hline $\mathrm{S} 17$ & 4-Арг-13 & Grab & K & $<$ & 2 & $\mathrm{mg} / \mathrm{L}$ \\
\hline$\$ 17$ & 4-Apr-13 & Grab & $\mathrm{Li}$ & $<$ & 0.01 & $\mathrm{mg} / \mathrm{L}$ \\
\hline $\mathrm{S} 17$ & 4-Apr-13 & Grab & $\mathrm{Mg}$ & & 14.5 & $\mathrm{mg} / \mathrm{L}$ \\
\hline$S 17$ & $4-\wedge p r-13$ & Grab & $\mathrm{Mn}$ & & 0.0159 & $\mathrm{mg} / \mathrm{L}$ \\
\hline$\$ 17$ & 4-Apr-13 & Grab & Mo & $<$ & 0.0004 & $\mathrm{mg} / \mathrm{L}$ \\
\hline$S / 7$ & 4-Apr-13 & Grab & $\mathrm{Na}$ & & 0.785 & $\mathrm{mg} / \mathrm{L}$ \\
\hline S17 & 4-Apr-13 & Grab & $\mathrm{Nb}$ & $<$ & 02 & $\mathrm{mg} / \mathrm{L}$ \\
\hline$\$ 17$ & 4-Apr-13 & Grab & $\mathrm{Ni}$ & $<$ & 0.002 & $\mathrm{mg} / \mathrm{L}$ \\
\hline$S / 7$ & 4-Арг-13 & Grab & $P$ & $<$ & 0.5 & $\mathrm{mg} / \mathrm{L}$ \\
\hline S17 & 4-Apr-13 & Grab & $\mathrm{Pb}$ & $<$ & 0.001 & $m g / L$ \\
\hline$\$ 17$ & 4-Apr-13 & Grab & $\mathrm{s}$ & & 1.80 & $\mathrm{mg} / \mathrm{L}$ \\
\hline$\$ 17$ & 4-Apr-13 & Grab & Sb & $<$ & 0.001 & $\mathrm{mg} / \mathrm{L}$ \\
\hline$\$ 17$ & 4-Арг-13 & Grab & $\mathrm{Se}$ & $<$ & 0.004 & $\mathrm{mg} / \mathrm{L}$ \\
\hline S17 & 4-Арг-13 & Grab & $\mathrm{Sr}$ & & 0.0464 & $\mathrm{mg} / \mathrm{L}$ \\
\hline S17 & 4-Apr-13 & Grab & Th & $<$ & 0.0002 & $\mathrm{mg} / \mathrm{L}$ \\
\hline S17 & 4-Apr-13 & Grab & $\mathrm{Ti}$ & $<$ & 0.05 & $\mathrm{mg} / \mathrm{L}$ \\
\hline$S 17$ & 4-Apr-13 & Grab & $\mathrm{Tl}$ & $<$ & 0.0002 & $\mathrm{mg} / \mathrm{L}$ \\
\hline S17 & 4-Apr-13 & Grab & $\mathrm{U}$ & & 0.000476 & $\mathrm{mg} / \mathrm{L}$ \\
\hline S17 & 4-Арт-13 & Grab & v & $<$ & 0.02 & $\mathrm{mg} / \mathrm{L}$ \\
\hline $\mathrm{S} 17$ & 4-Apr-13 & Grab & $\mathrm{Zn}$ & & 0.00253 & $\mathrm{mg} / \mathrm{L}$ \\
\hline S17 & 4-Apr-13 & Grab & $\mathrm{Z}_{\mathrm{r}}$ & $<$ & 0.2 & $\mathbf{m g} / \mathrm{L}$ \\
\hline
\end{tabular}




\section{Sector L \\ Outfall S17 \\ (continued)}

\begin{tabular}{|c|c|c|c|c|c|c|}
\hline Location & Date Collected & Samplc Type & Parameter & & Resulı & Units \\
\hline$S 17$ & $4-\wedge p r-13$ & Composite & $\mathrm{N}\left(\mathrm{C}^{\prime} \mathrm{a}\right) \mathrm{c}$ Tot $)$ & & 1.63 & $\mathrm{mg} / \mathrm{L}$ \\
\hline S17 & 4-Apr-13 & Composite & $\mathrm{NO} 3 / \mathrm{NO} 2 \mathrm{asN}$ & & 1.63 & $\mathrm{mg} / \mathrm{L}$ \\
\hline $\mathrm{S} 17$ & 4-Apr-13 & Composite & SuspSolids & & 1.00 & $\mathrm{mg} / \mathrm{L}$ \\
\hline S17 & 4-Apr-13 & Composite & Kjeldah|N & $<$ & 0.5 & $\mathrm{mg} / \mathrm{L}$ \\
\hline S17 & 4-Арг-13 & Compositc & $\mathrm{NH} 3 \mathrm{as} \mathrm{N}$ & $<$ & 0.2 & $\mathrm{mg} / \mathrm{L}$ \\
\hline $\mathrm{S} \perp 7$ & $4-A p r-13$ & Composite & $\mathrm{Ag}$ & $<$ & 0.0002 & $\mathrm{mg} / \mathrm{L}$ \\
\hline $\mathrm{S} 17$ & 4-Арг-13 & Composite & $\Lambda \mathrm{I}$ & $<$ & 0.2 & $\mathrm{mg} / \mathrm{L}$ \\
\hline $\mathrm{S} 17$ & 4-Арг-13 & Composite & As & $<$ & 0.002 & $\mathrm{mg} / \mathrm{L}$ \\
\hline $\mathrm{S} 17$ & 4-Apr-13 & Composite & B & $<$ & 0.1 & $\mathrm{mg} / \mathrm{L}$ \\
\hline $\mathrm{S} 17$ & 4-Apr- 13 & Composite & $\mathrm{Ba}$ & & 0.0490 & $\mathrm{mg} / \mathrm{L}$ \\
\hline $\mathrm{S} 17$ & 4-Apr-13 & Composite & $\mathrm{Be}$ & $<$ & 0.0002 & $\mathrm{mg} / \mathrm{L}$ \\
\hline S17 & 4-Apr- 13 & Composite & $\mathrm{Ca}$ & & 35.4 & $\mathrm{mg} / \mathrm{L}$ \\
\hline S17 & 4-Apr-13 & Composite & $\mathrm{Cd}$ & $<$ & 0.0002 & $\mathrm{mg} / \mathrm{L}$ \\
\hline S17 & 4-Apr- 13 & Compositc & Co & $<$ & 0.0002 & $\mathrm{mg} / \mathrm{L}$ \\
\hline S17 & 4-Арг-13 & Composite & $\mathrm{Cr}$ & $<$ & 0.002 & $\mathrm{mg} / \mathrm{L}$ \\
\hline S17 & $4-A p r-13$ & Composite & $\mathrm{Cu}$ & $<$ & 0.002 & $\mathrm{mg} / \mathrm{L}$ \\
\hline$\$ 17$ & 4-Арг-13 & Composite & $\mathrm{Fe}$ & & 0.0684 & $\mathrm{mg} / \mathrm{L}$ \\
\hline S17 & 4-Apr-13 & Composite & $k$ & $<$ & 2 & $\mathrm{mg} / \mathrm{L}$ \\
\hline S17 & $4-\Lambda$ pr-13 & Composite & $\mathrm{Li}$ & $<$ & 0.01 & $\mathrm{mg} / \mathrm{L}$ \\
\hline S17 & 4-Apr-13 & Composite & $\mathrm{Mg}$ & & 14.4 & $\mathrm{mg} / \mathrm{L}$. \\
\hline Si7 & 4-Aрг-13 & Composite & Mn & & 0.0118 & $\mathrm{mg} / \mathrm{L}$. \\
\hline S17 & 4-Арr-13 & Composite & Mo & $<$ & 0.0004 & $\mathrm{mg} / \mathrm{L}$ \\
\hline S17 & 4-Apr-13 & Composite & $\mathrm{Na}$ & & $0.84]$ & $\mathrm{mg} / \mathrm{L}$ \\
\hline S17 & 4-Apr-13 & Composite & $\mathrm{Nb}$ & $<$ & 0.2 & $\mathrm{mg} / \mathrm{L}$ \\
\hline $\mathrm{S} 17$ & 4-Apr-13 & Composite & $\mathrm{Ni}$ & $<$ & 0.002 & $\mathrm{mg} / \mathrm{L}$ \\
\hline S17 & 4-Apr-13 & Composite & $\mathrm{P}$ & $<$ & 0.5 & $\mathrm{mg} / \mathrm{L}$ \\
\hline S17 & $4-\Lambda p r-13$ & Composite & $\mathrm{Pb}$ & $<$ & $0.00 !$ & $\mathrm{mg} / \mathrm{L}$ \\
\hline S17 & 4-Apr-13 & Composite & $s$ & & 1.76 & $\mathrm{mg} / \mathrm{L}$ \\
\hline S17 & 4-Apr-13 & Composite & $\mathrm{Sb}$ & $<$ & 0.001 & $\mathrm{mg} / \mathrm{L}$ \\
\hline $\mathrm{S} 17$ & 4-Apr-13 & Composite & $\mathrm{Se}$ & $<$ & 0.004 & $\mathrm{mg} / \mathrm{L}$ \\
\hline$\$ 17$ & 4-Apr-13 & Composite & Sr & & 0.0455 & $\mathrm{mg} / \mathrm{L}$ \\
\hline S17 & 4-Арг-13 & Composite & Th & $<$ & 0.0002 & $\mathrm{mg} / \mathrm{l}$. \\
\hline S17 & 4-Apr-13 & Composite & $\mathrm{Ti}$ & $<$ & 0.05 & $\mathrm{mg} / \mathrm{L}$ \\
\hline S17 & 4-Apr-I3 & Composite & $\mathrm{Tl}$ & $<$ & 00002 & $\mathrm{mg} / \mathrm{L}$ \\
\hline S17 & 4-Арг-13 & Composite & $\mathrm{u}$ & & 0.000518 & $\mathrm{mg} / \mathrm{L}$ \\
\hline S17 & 4-Apr- 13 & Composite & V & $<$ & 0.02 & $\mathrm{mg} / \mathrm{L}$ \\
\hline $\mathrm{S} 17$ & 4-Apr-13 & Composite & $\mathrm{Zn}$ & & 0.00311 & $\mathrm{mg} / \mathrm{L}$ \\
\hline S17 & 4-Apr-13 & Composite & $\mathrm{Zr}$ & $<$ & 0.2 & $\mathrm{mg} / \mathrm{L}$ \\
\hline
\end{tabular}




\section{Sector N \\ Outfall S30}

\begin{tabular}{|c|c|c|c|c|c|c|}
\hline Location & Date Collected & Sample Type & Parameter & & Result & Units \\
\hline$\$ 30$ & $21-$ Sep-13 & Field & $\mathrm{pH}$ & & 8.1 & Std Units \\
\hline $\mathrm{S} 30$ & $21-S e p-13$ & Grab & HexExMatl & $<$ & 5.9 & $\mathrm{mg} / \mathrm{L}$ \\
\hline$\$ 30$ & 21-Sep-13 & Grab & SuspSolids & & 13.5 & $\mathrm{mg} / \mathrm{L}$ \\
\hline S30 & $21-$ Sep- 13 & Grab & $\mathrm{BOD}$ & & 5.15 & $\mathrm{mg} / \mathrm{L}$ \\
\hline$\$ 30$ & $21-$ Sep- 13 & Grab & COD & & 410 & $\mathrm{mg} / \mathrm{L}$ \\
\hline$\$ 30$ & $21-$ Sep-13 & Grab & $\wedge \mathrm{g}$ & & 0.000546 & $\mathrm{mg} / \mathrm{L}$ \\
\hline $\mathrm{S} 30$ & $21-\operatorname{Sep}-13$ & Girab & $\mathrm{Al}$ & & 0.878 & $\mathrm{mg} / \mathrm{L}$ \\
\hline$\$ 30$ & $21-$ Sep-13 & Grab & As & $<$ & 0.002 & $\mathrm{mg} / \mathrm{l}$. \\
\hline$\$ 30$ & $21-S e p-13$ & Grab & $B$ & $<$ & $0 . I$ & $\mathrm{mg} / \mathrm{L}$ \\
\hline$\$ 30$ & $21-\operatorname{Sep}-13$ & Grab & $\mathrm{Ba}$ & & 0.0625 & $\mathrm{mg} / \mathrm{L}$ \\
\hline$\$ 30$ & $21-\operatorname{Sep}-13$ & Grab & $\mathrm{Be}$ & $<$ & 0.0005 & $\mathrm{mg} / \mathrm{L}$. \\
\hline$S 30$ & $21-$ Sep- 13 & Grab & $\mathrm{Ca}$ & & 40.7 & $\mathrm{mg} / \mathrm{L}$ \\
\hline$\$ 30$ & 21-Sep-13 & Grab & $\mathrm{Cd}$ & $<$ & 0.0002 & $\mathrm{mg} / \mathrm{L}$ \\
\hline$\$ 30$ & $21-S e p-13$ & Grab & $\mathrm{Co}$ & & 0.000950 & $\mathrm{mg} / \mathrm{L}$ \\
\hline S30 & $21-$ Sep-13 & Grab & $\mathrm{Cr}$ & $<$ & 0.002 & $\mathrm{mg} / \mathrm{L}$ \\
\hline $\mathrm{S} 30$ & $21-$ Sep- 13 & Grab & $\mathrm{Cu}$ & & 0.00724 & $\mathrm{mg} / \mathrm{L}$ \\
\hline$\$ 30$ & $21-S e p-13$ & Grab & $\mathrm{Fe}$ & & 0.545 & $\mathrm{mg} / \mathrm{L}$ \\
\hline$\$ 30$ & $21-$ Sep- 13 & Grab & $\mathrm{Hg}$ & $<$ & 0.053 & $\mathrm{ug} / \mathrm{L}$ \\
\hline S30 & 21-Sep-13 & Grab & $k$ & & 2.77 & $\mathrm{mg} / \mathrm{L}$ \\
\hline S30 & $21-$ Sep- 13 & Grab & lit & $<$ & 0.01 & $\mathrm{mg} / \mathrm{L}$ \\
\hline$\$ 30$ & $21-$ Sep-13 & Grab & $\mathrm{Mg}$ & & 5.64 & $\mathrm{mg} / \mathrm{l}$ \\
\hline$\$ 30$ & 21-Sep-13 & Grab & $\mathrm{Mn}$ & & 0.0206 & $\mathrm{mg} / \mathrm{L}$ \\
\hline S30 & $21-$ Sep- 13 & Grab & Mo & & 0.00156 & $\mathrm{mg} / \mathrm{L}$ \\
\hline$\$ 30$ & $21-$ Sep-13 & Grab & $\mathrm{Na}$ & & 0.993 & $\mathrm{mg} / \mathrm{L}$ \\
\hline$\$ 30$ & $21-$ Sep- 13 & Grab & $\mathrm{Nb}$ & $<$ & 0.2 & $\mathrm{mg} / \mathrm{L}$ \\
\hline$\$ 30$ & $21-$ Sep- 13 & Grab & $\mathrm{Ni}$ & & 0.00580 & $\mathrm{mg} / \mathrm{L}$. \\
\hline$\$ 30$ & $21-\operatorname{Sep}-13$ & Grab & $\mathrm{P}$ & $<$ & 0.5 & $\mathrm{mmg} / \mathrm{L}$ \\
\hline $\mathrm{S} 30$ & $21-\mathrm{Sep}-13$ & Grab & $\mathrm{Pb}$ & & 0.00364 & $\mathrm{mg} / \mathrm{L}$ \\
\hline$\$ 30$ & $21-$ Sep- 13 & Grab & $S$ & & 19.4 & $\mathrm{mg} / \mathrm{L}$ \\
\hline S30 & $21-$ Sep-13 & Grab & $\mathrm{Sb}$ & $<$ & 0.001 & $\mathrm{mg} / \mathrm{L}$ \\
\hline$\$ 30$ & $21-$ Sep- 13 & Grab & $\mathrm{Se}$ & $<$ & 0.004 & $\mathrm{mg} / \mathrm{L}$ \\
\hline S30 & $21-$ Sep- 13 & Grab & $\mathrm{Sr}$ & & 0.129 & $\mathrm{mg} / \mathrm{L}$ \\
\hline $\mathrm{S} 30$ & $21-\operatorname{Sep}-13$ & Grab & Th & $<$ & 0.0002 & $\mathrm{mg} / \mathrm{L}$ \\
\hline $\mathrm{S} 30$ & $21-$ Sep-13 & Grab & $\mathrm{Ti}$ & $<$ & 0.05 & $\mathrm{mg} / \mathrm{L}$ \\
\hline$\$ 30$ & $2 \mid-$ Sep- 13 & Grab & 11 & $<$ & 0.0002 & $\mathrm{mg} / \mathrm{L}$ \\
\hline $\mathrm{S} 30$ & 21-Sep-13 & Grab & $\mathrm{v}$ & $<$ & 0.02 & $\mathrm{mg} / \mathrm{L}$ \\
\hline \$30 & 21-Sep-13 & Grab & $\mathrm{Zn}$ & & 0.0146 & $\mathrm{mg} / \mathrm{I}$. \\
\hline S30 & $21-S e p-13$ & Grab & 7. & $<$ & 0.2 & $\mathrm{mg} / \mathrm{L}$ \\
\hline
\end{tabular}




\section{Sector N \\ Outfall S30 \\ (continued)}

\begin{tabular}{|c|c|c|c|c|c|c|}
\hline Location & Date Collected & Sample Type & Parameter & & Result & Units \\
\hline$\$ 30$ & 21-Sep-13 & Grab & $\mathrm{U}$ & & 0.000490 & $\mathrm{mg} / \mathrm{L}$ \\
\hline S30 & 21-Sep-13 & Grab & U-234 & & 0098 & $\mathrm{pCi} / \mathrm{L}$ \\
\hline S30 & 2 l-Sep- 13 & Grab & $\mathrm{U}-235$ & & .0 .097 & $\mathrm{pCi} / \mathrm{L}$ \\
\hline$\$ 30$ & $21-\mathrm{Sep}-13$ & Grab & U-236 & & 0.019 & $\mathrm{pCi} / \mathrm{L}$ \\
\hline$\$ 30$ & 21-Sep-13 & Grab & $\mathrm{U}-238$ & & 0.16 & $\mathrm{pCi} / \mathrm{l}$ \\
\hline$\$ 30$ & 21-Sep-13 & Grab & $P C B$ &. & 0.5 & $\mathrm{ug} / \mathrm{L}$ \\
\hline$\$ 30$ & $21-\mathrm{Sep}-13$ & Grab & PCB-1016 & U' & 0.5 & $\operatorname{ug} / 1$. \\
\hline$\$ 30$ & $21-S e p-13$ & Grab & РCB-1221 & U & 0.5 & $\mathrm{ug} / \mathrm{L}$ \\
\hline S30 & 21-Sep-13 & Grab & PCB-1232 & () & 0.5 & $\mathrm{ug} / \mathrm{L}$ \\
\hline S30 & $21-S e p-13$ & Grab & РСB-1242 & $\mathrm{U}$ & 0.5 & $\mathrm{ug} / \mathrm{L}$ \\
\hline S30 & 21-Sep-13 & Grab & PCB-1248 & () & 0.5 & $\mathrm{ug} / \mathrm{L}$ \\
\hline S30 & $21-$ Sep-13 & Grab & PCB-1254 & $\mathrm{U}$ & 0.5 & $\mathrm{ug} / \mathrm{L}$ \\
\hline S30 & $21-$ Sep-13 & Grab & PCB- 1260 & $\mathrm{U}$ & 0.5 & $\mathrm{ug} / \mathrm{L}$ \\
\hline $\mathrm{S} 30$ & $21-S e p-13$ & Grab & PCB-1262 & U & 0.5 & $\mathrm{ug} / \mathrm{L}$ \\
\hline $\mathrm{S} 30$ & $21-S e p-13$ & Grab & PCB- 1268 & U & 0.5 & $11 \mathrm{~g} / \mathrm{L}$ \\
\hline$\$ 30$ & 21-Sep-13 & Grab & $\begin{array}{c}2.4 .5,6-\text { tetrach } / \text { oro- } \\
\text { m-xylene }\end{array}$ & & 0.56 & $u g / L$ \\
\hline S30 & $21-S e p-13$ & Grab & $\begin{array}{l}\text { Decachloro- } \\
\text { biphenyl }\end{array}$ & & 0.84 & $\mathrm{ug} / \mathrm{L}$ \\
\hline
\end{tabular}




\section{Sector N \\ Outfall S30 \\ (continued)}

\begin{tabular}{|c|c|c|c|c|c|c|}
\hline Location & Date Collected & Sample Type & Parameter & & Result & Units \\
\hline S30 & $21-$ Sep-13 & Composite & SuspSolids & & 11.5 & $\mathrm{mg} / \mathrm{L}$ \\
\hline$\$ 30$ & 21-Sep-13 & Composite & DissSolids & & 144 & $\mathrm{mg} / \mathrm{L}$ \\
\hline$\$ 30$ & $21-$-ер-13 & Composite & BOD & & 5.7 & $\mathrm{mg} / \mathrm{I}$, \\
\hline$\$ 30$ & $21-S c p-13$ & Composite & COD & & 43.0 & $\mathrm{mg} / \mathrm{l}$. \\
\hline$\$ 30$ & $21-\operatorname{Sep}-13$ & Composite & $\mathrm{Ag}$ & & 0.000386 & $\mathrm{mg} / \mathrm{L}$ \\
\hline$\$ 30$ & $21-$ Sep-13 & Composite & Al & & $0.9 \mid 4$ & $\mathrm{mg} / \mathrm{L}$ \\
\hline $\mathrm{S} 30$ & $21-S c p-13$ & Composite & As & $<$ & 0.002 & $\mathrm{mg} / \mathrm{L}$. \\
\hline$\$ 30$ & $2 \mid-\operatorname{Sep}^{-1} 3$ & Composite & $\mathrm{B}$ & $<$ & 0.1 & $\mathrm{mg} / \mathrm{L}$ \\
\hline$\$ 30$ & $21-$ Sep-13 & Composite & $\mathrm{Ba}$ & & 0.0493 & $\mathrm{mg} / \mathrm{L}$ \\
\hline S30 & $21 \cdot$ Sep-13 & Composite & $\mathrm{Be}$ & $<$ & 0.0005 & $\mathrm{mg} / \mathrm{L}$ \\
\hline$\$ 30$ & $21-$ Sep$^{-1} 3$ & Composite & $\mathrm{Ca}$ & & 30.3 & $\mathrm{mg} / \mathrm{L}$ \\
\hline$\$ 30$ & $21-$ Sep-13 & Composite & Cd & & 0.000210 & $\mathrm{mg} / \mathrm{L}$ \\
\hline S30 & $21 \cdot \operatorname{Sep}-13$ & Compositc & $\mathrm{Co}$ & & 0.000870 & $\mathrm{mg} / \mathrm{l}$. \\
\hline S30 & $21-$ Sep- 13 & Composite & $\mathrm{Cr}$ & $<$ & 0.002 & $\mathrm{mg} / \mathrm{L}$ \\
\hline$\$ 30$ & $21-$ Sep-13 & Composite & $\mathrm{Cu}^{\prime}$ & & 0.00679 & $\mathrm{mg} / \mathrm{L}$ \\
\hline $\mathrm{S} 30$ & $21-\operatorname{Sep}-13$ & Composite & $\mathrm{Fe}$ & & 0.540 & $\mathrm{mg} / \mathrm{L}$ \\
\hline $\mathrm{S} 30$ & $21-\operatorname{Sep}-13$ & Composite & $\mathrm{Hg}$ & $<$ & 0.054 & $\mathrm{ug} / \mathrm{L}$ \\
\hline$\$ 30$ & $21-\operatorname{Sep}-13$ & Compositc & $\mathrm{K}$ & & 2.67 & $\mathrm{mg} / \mathrm{L}$ \\
\hline$\$ 30$ & $2 \mid-\operatorname{Sep}-13$ & Composite & $\mathrm{Li}$ & $<$ & 0.01 & $\mathrm{mg} / \mathrm{I}$. \\
\hline$\$ 30$ & $21-$ Sep-13 & Composite & $\mathrm{Mg}$ & & 4.33 & $\mathrm{mg} / \mathrm{L}$ \\
\hline $\mathrm{S} 30$ & $2 \mid-\operatorname{Sep}-13$ & Composite & $\mathrm{Mn}$ & & $00|7|$ & $\mathrm{mg} / \mathrm{L}$ \\
\hline$\$ 30$ & 21-Sep-13 & Composite & Mo & & 0.00109 & $\mathrm{mg} / \mathrm{L}$ \\
\hline S30 & $2 J-S e p-13$ & Composite & $\mathrm{Na}$ & & 0.700 & $\mathrm{mg} / \mathrm{L}$ \\
\hline $\mathrm{S} 30$ & 2]-Sep-13 & Compositc & $\mathrm{Nb}$ & $<$ & 0.2 & $\mathrm{mg} / \mathrm{L}$. \\
\hline$\$ 30$ & $21-S e p-13$ & Composite & $\mathrm{Ni}$ & & 0.00478 & $\mathrm{mg} / \mathrm{L}$ \\
\hline$\$ 30$ & $21-$ Sep-13 & Composile & $\mathrm{P}$ & $<$ & 0.5 & $\mathrm{mg} / \mathrm{L}$ \\
\hline S30 & $21-S c p-13$ & Composite & $\mathrm{Pb}$ & & 0.00249 & $\mathrm{mg} / \mathrm{L}$ \\
\hline S30 & $2 \mid-\mathrm{Sep}-13$ & Composite & $\mathrm{S}$ & & 13.0 & $\mathrm{mg} / \mathrm{L}$ \\
\hline$\$ 30$ & $21-\operatorname{Sep}-13$ & Composite & Sb & $<$ & 0.001 & $\mathrm{mg} / \mathrm{L}$ \\
\hline$\$ 30$ & 2 I-Sep-13 & Composite & $\mathrm{Se}$ & $<$ & 0.004 & $\mathrm{mg} / \mathrm{L}$ \\
\hline $\mathrm{S} 30$ & $21-$ Sep-13 & Composite & Sr & & 0.0980 & $\mathrm{mg} / \mathrm{L}$ \\
\hline$\$ 30$ & 21 -Sep-1 3 & Composite & Th & $<$ & 0.0002 & $\mathrm{mg} / \mathrm{L}$ \\
\hline $\mathrm{S} 30$ & $21-$ Sep-13 & Composite & $\mathrm{Ti}$ & $<$ & 0.05 & $\mathrm{mg} / \mathrm{L}$ \\
\hline$\$ 30$ & $21-$ Sep-13 & Composite & $\mathrm{Tl}$ & $<$ & 0.0002 & $\mathrm{mg} / \mathrm{L}$. \\
\hline S30 & 2I-Sep-13 & Composite & V & $<$ & 0.02 & $\mathrm{mg} / \mathrm{L}$ \\
\hline$\$ 30$ & $21-$ Scp-13 & Composite & $\mathrm{Zn}$ & & 0.0156 & $\mathrm{mg} / \mathrm{L}$ \\
\hline$\$ 30$ & 2I-Sep-13 & Composite & 7. & $<$ & 0.2 & $\mathrm{mg} / \mathrm{L}$ \\
\hline
\end{tabular}




\section{Sector N \\ Outfall S30 \\ (continued)}

\begin{tabular}{|c|c|c|c|c|c|c|}
\hline Location & Date Collected & Sample Type & Parameter & & ResulI & Units \\
\hline $\mathrm{S} 30$ & $21-$ Sep-13 & Composite & U & & 0.000418 & $\mathrm{mg} / \mathrm{L}$ \\
\hline$\$ 30$ & $21-S e p-13$ & Composite & U-234 & & $\mathrm{N} / \mathrm{A}$ & w1 $\%$ \\
\hline$\$ 30$ & $21-$ Sep- 13 & Composite & $11235 \%$ & & $\mathrm{~N} / \mathrm{A}$ & wt $\%$ \\
\hline$\$ 30$ & $21-S e p-13$ & Composite & $\mathrm{U}-236$ & & $\mathrm{~N} / \mathrm{A}$ & $w 1 \%$ \\
\hline $\mathrm{S} 30$ & $21 \cdot \operatorname{Sep}-13$ & Composite & U-238 & & $\mathrm{N} / \mathrm{A}$ & $w t \%$ \\
\hline $\mathrm{S} 30$ & $21-\operatorname{Sep}-13$ & Compositc & U -235 & & 0.078 & $\mathrm{pCi} / \mathrm{L}$ \\
\hline$\$ 30$ & $21-\operatorname{Sep}-13$ & Composite & U -236 & & 0.0 & $p C i / L$ \\
\hline$\$ 30$ & $21-S_{-1}-13$ & Composite & U- 238 & & 0.95 & $\mathrm{pCi} / \mathrm{L}$ \\
\hline$\$ 30$ & 21-Sep-13 & Composite & U-234 & & 0.62 & $p \subset i / L$ \\
\hline$\$ 30$ & $21-\operatorname{Sep}-13$ & Composite & $\mathrm{PCB}$ & $U$ & 0.5 & $\mathrm{ug} / \mathrm{L}$ \\
\hline $\mathrm{S} 30$ & $2 \mid-\operatorname{Sep}-13$ & Composite & PCB-1016 & $\mathrm{U}$ & 0.5 & แg/L \\
\hline S30 & $21-$ Sep-13 & Composile & PCB-1221 & U & 0.5 & $\mathrm{ug} / \mathrm{L}$ \\
\hline$\$ 30$ & $21-\mathrm{Sep}-13$ & Composite & PCB-1232 & U & 0.5 & $\mathrm{ug} / \mathrm{L}$ \\
\hline $\mathrm{S} 30$ & $2]-S e p-13$ & Composile & PCB-1242 & U & 0.5 & $\mathrm{ug} / \mathrm{L}$ \\
\hline $\mathrm{s} 30$ & $21-$ Sep-13 & Composite & PCB- 1248 & U & 0.5 & $\mathrm{ug} / \mathrm{L}$ \\
\hline S30 & $21-S e p-13$ & Composite & PCB- 1254 & $\mathrm{U}$ & 0.5 & $\mathrm{ug} / \mathrm{L}$ \\
\hline $\mathrm{S} 30$ & $21-\operatorname{Sep}-13$ & Composite & PCB- 1260 & U & 0.5 & $u g / \mathrm{L}$ \\
\hline$\$ 30$ & $21-$ Sep-13 & Composite & PCB- 1262 & $\mathrm{U}$ & 0.5 & $\mathrm{ug} / \mathrm{L}$ \\
\hline $\mathrm{S} 30$ & $21-S e p-13$ & Composite & PCB-1268 & U! & 0.5 & $\mathrm{ug} / \mathrm{L}$ \\
\hline
\end{tabular}


Appendix C

2013 Storm Water Analytical Results, In-Stream Monitoring Locations and Major Outfalls 


\section{In-Stream Monitoring Location $\mathrm{C03}$}

\begin{tabular}{|c|c|c|c|c|c|c|}
\hline Location & Date Collecled & Sample Type & Parameter & & Result & Units \\
\hline $\mathrm{C} 03$ & 18-Mar-13 & Field & $\mathrm{pHI}$ & & 8.1 & Std Units \\
\hline $\mathrm{CO3}$ & 18-Mar-13 & Grab & Es coli & & 22 & $\mathrm{col} / 100 \mathrm{ml}$ \\
\hline $\mathrm{CO}$ & 18-Mar-13 & Grab & [texExMatl & $<$ & 6.2 & $m \underline{g} / 1$ \\
\hline $\mathrm{CO}$ & 18-Маг-13 & Composite & SuspSolids & & 18.5 & $\mathrm{mg} / \mathrm{L}$ \\
\hline $\mathrm{CO}$ & 18-Mar-13 & Composite & KjeldahlN & $<$ & 0.5 & $\mathrm{mg} / \mathrm{L}$ \\
\hline $\mathrm{CO3}$ & 18-Mar-13 & Composite & Surfactants & $<$ & 0.10 & $\mathrm{mg} / \mathrm{l}$. \\
\hline $\mathrm{C} 03$ & $18-\mathrm{Mar}-13$ & Composite & $\mathrm{NO} 3 / \mathrm{NO} 2 \mathrm{as} \mathrm{N}$ & & 1.53 & $\mathrm{mg} / \mathrm{L}$ \\
\hline $\mathrm{C} 03$ & 18-Mar-13 & Composite & $\mathrm{Ag}$ & $<$ & 0.0002 & $\mathrm{mg} / \mathrm{L}$ \\
\hline $\mathrm{CO3}$ & $18 \cdot \mathrm{Mar}-13$ & Composite & $\mathrm{Al}$ & & 0.591 & $\mathrm{mg} / \mathrm{l}$. \\
\hline $\mathrm{C} 03$ & 18-Mar-13 & Composite & As & $<$ & 0.002 & $\mathrm{mg} / \mathrm{L}$ \\
\hline $\mathrm{C} 03$ & 18 -Mar-13 & Composite & $\mathrm{B}$ & $<$ & 0.1 & $\mathrm{mg} / \mathrm{L}$ \\
\hline $\mathrm{C} 03$ & $18-\mathrm{Mar}-13$ & Composite & $\mathrm{Ba}$ & & 0.0432 & $\mathrm{mg} / \mathrm{L}$ \\
\hline $\mathrm{C} 03$ & 18-Mar-13 & Composite & $\mathrm{Bc}$ & $<$ & 0.0002 & $\mathrm{mg} / \mathrm{L}$ \\
\hline $\mathrm{C} 03$ & $18-$ Mar-13 & Composite & $\mathrm{Ca}$ & & 34.8 & $\mathrm{mg} / \mathrm{L}$ \\
\hline $\mathrm{C} 03$ & 18-Mar-I3 & Composite & $\mathrm{Cd}$ & & 0000290 & $\mathrm{mg} / \mathrm{L}$ \\
\hline $\mathrm{CO3}$ & 18-Mar-13 & Composite & Co & & 0.000512 & $\mathrm{mg} / \mathrm{l}$. \\
\hline $\mathrm{C} 03$ & $18-$ Mar- 13 & Composite & $\mathrm{Cr}$ & & 0.00247 & $\mathrm{mg} / \mathrm{L}$ \\
\hline $\mathrm{C} 03$ & 18-Mar-13 & Composite & $\mathrm{Cu}$ & & 0.0114 & $\mathrm{mg} / \mathrm{L}$. \\
\hline $\mathrm{CO}$ & 18-Mar-13 & Composite & re & & 0.605 & $\mathrm{mg} / \mathrm{L}$ \\
\hline $\mathrm{C} 03$ & 18 -Mar-13 & Composite & $\mathrm{Hg}$ & & 0.000901 & $\mathrm{mg} / \mathrm{L}$ \\
\hline $\mathrm{C} 03$ & 18 -Mar-13 & Composite & k & $<$ & 2 & $\mathrm{mg} / \mathrm{L}$ \\
\hline $\mathrm{C} 03$ & 18-Mar-13 & Composite & $\mathrm{Li}$ & & 0.0308 & $\mathrm{mg} / \mathrm{L}$ \\
\hline $\mathrm{C} 03$ & 18-Mar-13 & Composite & $\mathrm{Mg}$ & & 8.94 & $\mathrm{mg} / \mathrm{L}$ \\
\hline $\mathrm{C} 03$ & 18-Mar-13 & Composite & $\mathrm{Mn}$ & & 0.0776 & $\mathrm{mg} / \mathrm{L}$ \\
\hline $\mathrm{C}^{\prime} 03$ & 18 -Mar-13 & Composite & Mo & & 0.00456 & $\mathrm{mg} / \mathrm{L}$ \\
\hline $\mathrm{C} 03$ & 18 -Маг-13 & Composite & $\mathrm{Na}$ & & 24.9 & $\mathrm{mg} / \mathrm{L}$ \\
\hline $\mathrm{C} 03$ & 18-Mar-13 & Composite & $\mathrm{Nb}$ & $<$ & 02 & $\mathrm{mg} / \mathrm{L}$. \\
\hline $\mathrm{C} 03$ & 18-Mar-13 & Composite & $\mathrm{Ni}$ & & 0.00205 & $\mathrm{mg} / \mathrm{L}$ \\
\hline $\mathrm{C} 03$ & 18-Мar-13 & Composite & $P$ & $<$ & 0.5 & $\mathrm{mg} / \mathrm{L}$ \\
\hline $\mathrm{CO3}$ & 18-Mar-13 & Composite & $\mathrm{Pb}$ & & $0.0035 \mathrm{I}$ & $\mathrm{mg} / \mathrm{L}$ \\
\hline $\mathrm{C} 03$ & $18-\mathrm{Mar}-13$ & Composite & $\mathrm{S}$ & & 7.21 & $\mathrm{mg} / \mathrm{L}$ \\
\hline $\mathrm{C} 03$ & 18-Мar-13 & Composite & Sb & $<$ & 0.001 & $\mathrm{mg} / \mathrm{L}$ \\
\hline $\mathrm{C} 03$ & 18-Mar-13 & Composite & $\mathrm{Se}$ & $<$ & 0.004 & $\mathrm{mg} / \mathrm{L}$ \\
\hline $\mathrm{C} 03$ & 18-Mar-13 & Composite & $\mathrm{Sr}$ & & 0.0946 & $\mathrm{mg} / \mathrm{L}$ \\
\hline $\mathrm{C} 03$ & $18-$ Маг-13 & Composite & Th & $<$ & 0.0002 & $\mathrm{mg} / \mathrm{L}$ \\
\hline $\mathrm{C} 03$ & 18-Маг-І3 & Composite & $\mathrm{Ti}$ & $<$ & 0.05 & $\mathrm{mg} / \mathrm{L}$ \\
\hline $\mathrm{C} 03$ & $18-$ Mar-13 & Composite & $\mathrm{Tl}$ & $<$ & 0.0002 & $\mathrm{mg} / \mathrm{L}$ \\
\hline $\mathrm{CO} 3$ & $18-$ Маг-13 & Composile & v & $<$ & 0.02 & $\mathrm{mg} / \mathrm{L}$ \\
\hline $\mathrm{CO3}$ & $18-\mathrm{Mar}-13$ & Composile & $\mathrm{Zn}$ & & 0.0597 & $\mathrm{mg} / \mathrm{L}$ \\
\hline $\mathrm{CO}$ & 18-Мar-13 & Composite & $\mathrm{Zr}$ & $<$ & 0.2 & $\mathrm{mg} / \mathrm{L}$ \\
\hline
\end{tabular}




\section{In-Stream Monitoring \\ Location $\mathrm{C03}$ \\ (continued)}

\begin{tabular}{|c|c|c|c|c|c|c|}
\hline Location & Date Collected & Sample Type & Parameter & & Result & Units \\
\hline $\mathrm{C03}$ & 18-Mar-13 & Composite & $\overline{\mathrm{PCB}}$ & $\mathrm{U}$ & 0.53 & $\mathrm{ug} / \mathrm{L}$ \\
\hline $\mathrm{CO3}$ & I8-Mar-I3 & Composite & РСB-1016 & $\mathrm{U}$ & 0.53 & ug $/ \mathrm{L}$ \\
\hline $\mathrm{C} 03$ & 18-Mar-13 & Composite & PCB-1221 & $\mathrm{U}$ & 0.53 & ug/L \\
\hline $\mathrm{CO3}$ & $18-$ Mar- 13 & Composite & PCB- 1232 & (1) & 0.53 & $u g / L$ \\
\hline $\mathrm{C} 03$ & 18-Mar-13 & Composite & PCB- 1242 & U & 0.53 & $\mathrm{tg} / \mathrm{L}$ \\
\hline $\mathrm{C} 03$ & $18-$ Мат-13 & Composite & PCB-1248 & U & 0.53 & $\mathrm{ug} / \mathrm{L}$ \\
\hline $\mathrm{C} 03$ & 18-Mar-13 & Composite & PCB- 1254 & U & 0.53 & $\mathrm{ug} / \mathrm{L}$ \\
\hline $\mathrm{C} 03$ & 18-Mar-13 & Composite & PCB- 1260 & $\mathrm{U}$ & 0.53 & $\mathrm{ug} / \mathrm{L}$. \\
\hline $\mathrm{C} 03$ & 18-Mar-13 & Composite & $P C B \cdot 1262$ & U & 0.53 & $\mathrm{ug} / \mathrm{L}$ \\
\hline $\mathrm{CO3}$ & 18-Mar-13 & Composite & PCB-1268 & U & 0.53 & $\mathrm{ug} / \mathrm{L}$ \\
\hline $\mathrm{C} 03$ & 18-Mar-13 & Composite & U & & 0.0137 & $\mathrm{mg} / \mathrm{L}$ \\
\hline $\mathrm{CO}$ & 18-Mar-13 & Composite & U-234 & $<$ & 0.005 & wt $\%$ \\
\hline $\mathrm{C} 03$ & 18-Mar-I3 & Composite & U235\% & & 0.373 & wt $\%$ \\
\hline $\mathrm{CO} 3$ & 18-Mar-I3 & Composite & $\mathrm{U}-236$ & & 0.0206 & wt $\%$ \\
\hline $\mathrm{CO}$ & 18-Mar-13 & Composite & U-238 & & 99.6 & $w \%$ \\
\hline
\end{tabular}




\section{In-Stream Monitoring \\ Location C11 \\ (continued)}

\begin{tabular}{|c|c|c|c|c|c|c|}
\hline Location & Date Collected & Sample Type & Parameter & & Resuit & Units \\
\hline CII & 18-Mar-13 & Composite & U & & 0.0164 & $\mathrm{mg} / \mathrm{L}$ \\
\hline CII & 18-Маг-13 & Composite & U. 234 & $<$ & 0.005 & $w ! \%$ \\
\hline $\mathrm{C} 11$ & 18-Маг-13 & Composilc & U235\% & & 0356 & $w \%$ \\
\hline $\mathrm{CII}$ & 18-Mar-13 & Composilc & UJ-236 & $<$ & 0.005 & $w 1 \%$ \\
\hline C11 & 18-Маг-13 & Composite & U-238 & & 99.6 & wt $\%$ \\
\hline $\mathrm{Cll}$ & $18-M a r-13$ & Composite & PCB & [ ] & 0.53 & $\mathrm{ug} / \mathrm{L}$ \\
\hline C11 & 18-Mar-13 & Composite & PCB-1016 & $\mathrm{U}$ & 0.53 & $\mathrm{Ig} / \mathrm{L}$ \\
\hline $\mathrm{Cll}$ & 18-Mar-13 & Composite & PCB-1221 & II & 0.53 & $\mathrm{ug} / \mathrm{L}$ \\
\hline CII & 18-Mar-13 & Composite & PCB-1232 & [ ] & 0.53 & $\mathrm{ug} / \mathrm{L}$ \\
\hline CII & 18-Маг-13 & Composite & PCB-1 242 & $\mathrm{U}$ & 0.53 & $\mathrm{ug} / \mathrm{l}$. \\
\hline $\mathrm{Cll}$ & 18-Mar-13 & Composite & PCB-1248 & II & 0.53 & $\mathrm{ug} / \mathrm{L}$ \\
\hline $\mathrm{Cll}$ & $18-M a r-13$ & Composite & PCB- 1254 & U & 0.53 & $\mathrm{ug} / \mathrm{L}$ \\
\hline C11 & 18-Мar-13 & Composite & PCB- -260 & $\mathrm{U}$ & 0.53 & $\mathrm{ug} / \mathrm{l}$. \\
\hline C11 & $18-\mathrm{Mar}-13$ & Compositc & PCB-1262 & U & 0.53 & $\mathrm{ug} / \mathrm{L}$ \\
\hline C'11 & 18-Мar- 3 & Composite & PCB- 1268 & $\mathrm{U}$ & 0.53 & $\mathrm{ug} / \mathrm{L}$ \\
\hline
\end{tabular}




\section{Raw Water Flow Augmentation}

\begin{tabular}{|c|c|c|c|c|c|c|}
\hline Location & Date Collected & Sample Type & Parameter & & Result & Units \\
\hline RAWH2O & 18-Mar-13 & Composite & KjeldahlN & $<$ & 0.5 & $\mathrm{mg} / \mathrm{L}$ \\
\hline RAWH2O & $18-\mathrm{Mar}-13$ & Composite & Surfactants & $<$ & 0.10 & $\mathrm{mg} / \mathrm{L}$ \\
\hline RAWH2O & $18-M a r-13$ & Composite & $\mathrm{NO} 3 / \mathrm{NO} 2 \mathrm{asN}$ & & 0.491 & $\mathrm{mg} / \mathrm{L}$ \\
\hline RAWH $2 O$ & 18-Mar-13 & Composite & SuspSolids & $<$ & 1.0 & $\mathrm{mg} / \mathrm{L}$ \\
\hline $\mathrm{R} \wedge \mathrm{WH} 2 \mathrm{O}$ & 18-Mar-13 & Composite & $\Lambda \mathrm{g}$ & $<$ & 0.0002 & $\mathrm{mg} / \mathrm{l}$, \\
\hline RAWI12O & I8-Mar-13 & Compositc & $\mathrm{Al}$ & $<$ & 02 & $\mathrm{mg} / \mathrm{L}$ \\
\hline RAWH2O & 18-Mar-13 & Composite & As & $<$ & 0.002 & $\mathrm{mg} / \mathrm{L}$ \\
\hline RAWI120 & I8-Mar-13 & Composite & B & $<$ & 0.1 & $\mathrm{mg} / \mathrm{L}$ \\
\hline$R A W: 120$ & $18-M a r-13$ & Compositc & $\mathrm{Ba}$ & & 0.0317 & $\mathrm{mg} / \mathrm{L}$ \\
\hline $\mathrm{R} \wedge \mathrm{WH} 2 \mathrm{O}$ & 18-Mar-13 & Composite & $\mathrm{Be}$ & $<$ & 0.0002 & $\mathrm{mg} / \mathrm{L}$ \\
\hline RAWI-12O & 18-Mar-13 & Composite & $\mathrm{Ca}$ & & 36.1 & $\mathrm{mg} / \mathrm{L}$ \\
\hline $\mathrm{R} \wedge \mathrm{WH} 2 \mathrm{O}$ & $18-M a r-13$ & Composite & $\mathrm{Cd}$ & $<$ & 0.0002 & $\mathrm{mg} / \mathrm{l}$. \\
\hline RAWH 20 & 18-Маг-13 & Composite & $\mathrm{Co}$ & $<$ & 0.0002 & $\mathrm{mg} / \mathrm{l}$. \\
\hline RAWHL2O & 18-Mar-13 & Composite & $\mathrm{Cr}$ & $<$ & 0.002 & $\mathrm{mg} / \mathrm{L}$. \\
\hline RAWH2O & 18-Маг-13 & Composite & $\mathrm{Cu}$ & $<$ & 0.002 & $\mathrm{mg} / \mathrm{L}$ \\
\hline RAWIIZO & $18-\mathrm{Mar}-13$ & Composite & le & & 0.135 & $m g / L$ \\
\hline RAWH 20 & 18-Mar-13 & Composite & $\mathrm{Hg}$ & $<$ & 0.053 & $\mathrm{ug} / \mathrm{L}$ \\
\hline RAWH2O & 18-Mar-13 & Composite & K & $<$ & 2 & $\mathrm{mg} / \mathrm{l}$. \\
\hline RAWH2O & 18-Mar-13 & Composite & $1 . i$ & $<$ & 0.01 & $\mathrm{mg} / \mathrm{L}$ \\
\hline RAWH 120 & 18-Mar-13 & Composite & $\mathrm{Mg}$ & & 10.3 & $\mathrm{mg} / \mathrm{L}$ \\
\hline RAWH2O & 18-Mar-13 & Composite & $\mathrm{Mn}$ & & 0.0217 & $\mathrm{mg} / \mathrm{L}$ \\
\hline RAWH2O & 18-Mar-13 & Composite & Mo & $<$ & 0.0004 & $\mathrm{mg} / \mathrm{L}$ \\
\hline RAWI 120 & 18-Mar- 3 & Composite & $\mathrm{Na}$ & & 5.63 & $\mathrm{mg} / \mathrm{I}$. \\
\hline RAWII2O & $18-$ Mar-13 & Composite & $\mathrm{Nb}$ & $<$ & 02 & $\mathrm{mg} / \mathrm{l}$. \\
\hline RAWII2O & $18-M a r-13$ & Composite & $\mathrm{Ni}$ & $<$ & 0.002 & $\mathrm{mg} / \mathrm{l}$. \\
\hline RAWH2O & $18-M a r-13$ & Composite & $P$ & $<$ & 0.5 & $\mathrm{mg} / \mathrm{l}$. \\
\hline RAWH2O & 18 -Mar-13 & Composite & $\mathrm{Pb}$ & $<$ & 0.001 & $\mathrm{mg} / \mathrm{l}$. \\
\hline $\mathrm{RAWH} 2 \mathrm{O}$ & 18-Мar-13 & Composite & $S$ & & $7.6 \mathrm{l}$ & $\mathrm{mg} / \mathrm{L}$ \\
\hline RAWH2O & 18-Mar-13 & Composite & Sb & $<$ & 0.001 & $\mathrm{mg} / \mathrm{L}$ \\
\hline RAWH2O & $18-M a r-13$ & Composite & $\mathrm{Se}$ & $<$ & 0.004 & $\mathrm{mg} / \mathrm{L}$ \\
\hline RAWH2O & 18-Mar-। 3 & Composite & $\mathrm{Sr}$ & & 0.110 & $\mathrm{mg} / \mathrm{L}$ \\
\hline RAWH2O & 18-Mar-I3 & Composite & Th & $<$ & 0.0002 & $\mathrm{mg} / \mathrm{L}$ \\
\hline RAWH $2 O$ & 18-Мar-I3 & Composite & $\mathrm{Ti}$ & $<$ & 0.05 & $\mathrm{mg} / \mathrm{L}$. \\
\hline RAWH 20 & 18-Mar-13 & Composite & T) & $<$ & 0.0002 & $\mathrm{mg} / \mathrm{L}$ \\
\hline RAWH $2 O$ & 18-Mar-13 & Composite & $\mathrm{v}$ & $<$ & 0.02 & $\mathrm{mg} / \mathrm{L}$ \\
\hline $\mathrm{R} \wedge \mathrm{WH} 2 \mathrm{O}$ & 18-Mar-13 & Composite & $\mathrm{Zn}$ & & 0.00276 & $\mathrm{mg} / \mathrm{L}$ \\
\hline $\mathrm{RAWH} 2 \mathrm{O}$ & $18-\mathrm{Mar}-13$ & Composite & $\mathrm{Zr}$ & $<$ & 0.2 & $\mathrm{mg} / \mathrm{L}$ \\
\hline
\end{tabular}




\section{Raw Water Flow Augmentation (continued)}

\begin{tabular}{|c|c|c|c|c|c|c|}
\hline Location & Datc Collected & Sample Type & Parameler & & Resuli & Units \\
\hline RAWH2O & $18-M a r-13$ & Composite & $\mathrm{U}$ & & 0.000212 & $\mathrm{mg} / \mathrm{L}$ \\
\hline RAWH2O & $18-M^{2}-13$ & Composite & $\mathrm{U}-234$ & & $\mathrm{~N} / \mathrm{A}$ & wt $\%$ \\
\hline RAWHI2O & $18-\mathrm{Mar}-13$ & Composite & $\mathrm{U} 235 \%$ & & N/A & wt $\%$ \\
\hline RAW 120 & 18-Mar-13 & Composite & $U-236$ & & $N / A$ & wt $\%$ \\
\hline $\mathrm{RAWH} 2 \mathrm{O}$ & I8-Mar-13 & Composite & U-238 & & $N / A$ & w1 $\%$ \\
\hline RAWII2O & 18-Mar-13 & C'omposite & PCB & U & 0.46 & $\mathrm{ug} / \mathrm{L}$ \\
\hline RAWH2O & 18-Mar-13 & Composite & PCB-1016 & $\mathrm{U}$ & 046 & $\mathrm{ug} / \mathrm{L}$ \\
\hline RAWII2O & $18-\mathrm{Mar}-13$ & Composite & PCB- 1221 & $\mathrm{U}$ & 0.46 & $\mathrm{ug} / \mathrm{s}$. \\
\hline $\mathrm{R} A \mathrm{WH} 2 \mathrm{O}$ & $18-M a r-13$ & Composite & PCB- 1232 & II & 0.46 & $\mathrm{ug} / \mathrm{L}$ \\
\hline $\mathrm{RAWH} 2 \mathrm{O}$ & $18-\mathrm{Mar}-13$ & Composite & $\mathrm{PCB}-1242$ & $\mathrm{U}$ & 0.46 & $\mathrm{ug} / \mathrm{L}$ \\
\hline $\mathrm{RAWH} 2 \mathrm{O}$ & $18-$ Mar-13 & Composite & PCB- 1248 & U & 0.46 & $\mathrm{ug} / \mathrm{L}$ \\
\hline RAWH $12 \mathrm{O}$ & $18-\mathrm{Mar}-13$ & Composite & PCB- 1254 & $\mathrm{U}$ & 0.46 & $\mathrm{ug} / \mathrm{l}$, \\
\hline $\mathrm{RAWH} 2 \mathrm{O}$ & $18-M a r-13$ & Composite & PCB- 1260 & U & 0.46 & $\mathrm{ug} / \mathrm{L}$ \\
\hline RAWH $2 O$ & $18-M a r-13$ & Composite & PCB-1262 & $\mathrm{U}$ & 0.46 & $\mathrm{ug} / \mathrm{L}$ \\
\hline RAWH2O & $18-\mathrm{Mar}-13$ & Composite & РCB-1268 & [] & 0.46 & $\mathrm{ug} / \mathrm{L}$ \\
\hline
\end{tabular}




\section{Major Outfall 021}

\begin{tabular}{|c|c|c|c|c|c|c|}
\hline Location & Date Collected & Sample type & Parameter & & Result & Units \\
\hline 021 & 18-Mar-13 & Field & $\mathrm{pH}$ & & 7.9 & Std Units \\
\hline 021 & $18-\mathrm{Mar}-13$ & Grab & Lis coli & & 210 & $\mathrm{col} / 100 \mathrm{ml}$ \\
\hline 021 & 18-Mar-13 & Grab & HexExMat1 & $<$ & 5.7 & $\mathrm{mg} / \mathrm{L}$ \\
\hline 021 & 18-Mar-1 3 & Grab & SuspSolids & & 66.5 & $\mathrm{mg} / \mathrm{L}$ \\
\hline 021 & 18-Mar-13 & Grab & Surfactants & & 0.255 & $\mathrm{mg} / \mathrm{L}$ \\
\hline 021 & 18 -Mar- 13 & Grab & KjeldahlN & & 0.582 & $\mathrm{mg} / \mathrm{L}$ \\
\hline 021 & 18-Mar-13 & Grab & $\mathrm{NO} 3 / \mathrm{NO} 2 \mathrm{asN}$ & & 0.975 & $\mathrm{mg} / \mathrm{L}$ \\
\hline 021 & $18-\mathrm{Mar}-13$ & Grab & $N(C a l c T o t)$ & & 1.56 & $\mathrm{mg} / \mathrm{L}$ \\
\hline 021 & 18-Mar-13 & Grab & $\Lambda g$ & & 0.000206 & $\mathrm{mg} / \mathrm{I}$, \\
\hline 021 & $18-\mathrm{Mar}=13$ & Grab & Al & & 2.27 & $\mathrm{mg} / \mathrm{L}$ \\
\hline 021 & 18-Mar-13 & Grab & $A S$ & $<$ & 0.002 & $\mathrm{mg} / \mathrm{l}$. \\
\hline $02 !$ & $18-M a r-13$ & Grab & $B$ & $<$ & 0.1 & $\mathrm{mg} / \mathrm{L}$ \\
\hline 021 & $18-\mathrm{Mar}-13$ & Grab & $\mathrm{Ba}$ & & 0.129 & $\mathrm{mg} / \mathrm{L}$ \\
\hline 021 & 18-Mar-13 & Grab & $\mathrm{Be}$ & $<$ & 0.0002 & $\mathrm{mg} / \mathrm{L}$ \\
\hline 021 & $18-\mathrm{Mar}-13$ & Grab & $\mathrm{Ca}$ & & 57 & $\mathrm{mg} / \mathrm{L}$ \\
\hline 021 & 18-Mar-I3 & Grab & Cd & $<$ & 0.0002 & $\mathrm{mg} /$. \\
\hline 021 & $18-\mathrm{Mar}-13$ & Grab & Co & & 0.00191 & $\mathrm{mg} / \mathrm{L}$ \\
\hline 021 & $18-\mathrm{Mar}-13$ & Grab & $\mathrm{Cr}$ & & 0.0065 & $\mathrm{mg} / \mathrm{L}$ \\
\hline 021 & $18-\mathrm{Mar}-13$ & Grab & $\mathrm{Cu}$ & & 0.033 & $\mathrm{mg} / \mathrm{l}$ \\
\hline 021 & 18-Mar-13 & Grab & $\mathrm{Fe}$ & & 4.11 & $\mathrm{mg} / \mathrm{L}$ \\
\hline 021 & 18-Mar-13 & Grab & $11 \mathrm{~g}$ & & 0.000253 & $\mathrm{mg} / \mathrm{L}$ \\
\hline 021 & 18-Mar-13 & Grab & $\mathrm{K}$ & & 4.3 & $\mathrm{mg} / \mathrm{L}$ \\
\hline 021 & 18-Mar-13 & Grab & Li & $<$ & 0.01 & $\mathrm{mg} / \mathrm{L}$ \\
\hline 021 & 18-Mar-13 & Grab & $\mathrm{Mg}$ & & 11.6 & $\mathrm{mg} / \mathrm{l}$ \\
\hline 021 & | 8-Mar-13 & Grab & $\mathrm{Mn}$ & & 0.239 & $\mathrm{mg} / \mathrm{l}$ \\
\hline 021 & 18-Mar- 13 & Grab & Mo & & 0.00258 & $\mathrm{mg} / \mathrm{L}$ \\
\hline 021 & 18-Mar-13 & Grab & $\mathrm{Na}$ & & 102 & $\mathrm{mg} / \mathrm{L}$ \\
\hline 021 & 18-Mar-13 & Grab & $\mathrm{Nb}$ & $<$ & 0.2 & $\mathrm{mg} / \mathrm{L}$ \\
\hline 021 & $18-M a r-13$ & Grab & $\mathrm{Ni}$ & & 0.00458 & $\mathrm{mg} / \mathrm{L}$ \\
\hline $02 \mathrm{t}$ & 18-Mar-13 & Grab & $\mathrm{p}$ & $<$ & 0.5 & $\mathrm{mg} / \mathrm{L}$ \\
\hline $02 !$ & 18-Mar-13 & Grab & $\mathrm{Pb}$ & & 0.00572 & $\mathrm{mg} / \mathrm{L}$ \\
\hline 021 & 18-Mar-13 & Grab & $\mathrm{S}$ & & 12.5 & $\mathrm{mg} / \mathrm{L}$ \\
\hline 021 & 18 -Mar- 13 & Grab & Sb & $<$ & 0.001 & $\mathrm{mg} / \mathrm{l}$ \\
\hline 021 & 18-Мar-13 & Grab & Se & $<$ & 0.004 & $\mathrm{mg} / \mathrm{L}$ \\
\hline 021 & 18-Mar-13 & Grab & $\mathrm{Sr}$ & & 0.157 & $\mathrm{mg} / \mathrm{L}$ \\
\hline 021 & $18-\mathrm{Mar}-13$ & Grab & Th & & 0.00033 & $\mathrm{mg} / \mathrm{l}$ \\
\hline 021 & 18-Mar-13 & Grab & $\mathrm{Ti}$ & & 0.0965 & $m g / L$ \\
\hline 021 & 18-Mar-13 & Grab & $\mathrm{T} 1$ & $<$ & 0.0002 & $\mathrm{mg} / \mathrm{L}$ \\
\hline 021 & 18-Mar-13 & Grab & U & & 0.00694 & $\mathrm{mg} / \mathrm{L}$ \\
\hline 021 & 18-Mar-13 & Grab & $\mathrm{v}$ & $<$ & 0.02 & $\mathrm{mg} / \mathrm{L}$ \\
\hline 021 & I 8-Mar-13 & Grab & $\mathrm{Zn}$ & & 0.109 & $\mathrm{mg} / \mathrm{L}$ \\
\hline 021 & 18-Mar- 13 & Grab & $\mathrm{Zr}$ & $<$ & 0.2 & $\mathrm{mg} / \mathrm{L}$ \\
\hline
\end{tabular}




\section{Major Outfall 021 \\ (continued)}

\begin{tabular}{|c|c|c|c|c|c|c|}
\hline Location & Datc Collected & Sample type & Рarameter & & Resull & Units \\
\hline 021 & 18-Mar-13 & Grab & Alpha & & 86 & $\mathrm{nCi} / \mathrm{L}$ \\
\hline 021 & 18-Маг-13 & Grab & Beta & & 14 & $\mathrm{nC} 1 / \mathrm{I}$. \\
\hline 021 & 18-Mar-13 & Grab & U & & 0.0056 & $\mathrm{mg} / \mathrm{L}$ \\
\hline 021 & 18-Mar-13 & Grab & $U-234$ & $<$ & 0.005 & wt $\%$ \\
\hline 021 & 18-Мar-I3 & Grab & U $235 \%$ & & 1.01 & wt $\%$ \\
\hline 021 & 18-Mar-13 & Grab & U-236 & & 0.00763 & w1 $\%$ \\
\hline 021 & 18-Mar-13 & Grab & U-238 & & 99 & wit $\%$ \\
\hline 021 & 18-Mar-I3 & Grab & $\mathrm{U}-235$ & & 0.064 & $\mathrm{pCi} / \mathrm{L}$ \\
\hline 021 & 18-Mar.13 & Grab & $\mathrm{U}-236$ & & $0.0000 \mid$ & $\mathrm{pCi} / \mathrm{L}$. \\
\hline 021 & 18-Mar-13 & Grab & $\mathrm{U}-238$ & & 1.4 & $\mathrm{pCi} / \mathrm{L}$ \\
\hline 021 & 18-Mar-13 & Grab & $U-234$ & & 18 & $\mathrm{pC} \mathrm{Ci} / \mathrm{L}$ \\
\hline 021 & 18-Mar-13 & Grab & $\mathrm{PCB}$ & . & 0088 & $\operatorname{tg} / \mathrm{L}$ \\
\hline 021 & 18-Mar-13 & Grab & PCB-1016 & $\mathrm{U}$ & 0.5 & $\mathrm{ug} / \mathrm{L}$ \\
\hline 021 & 18-Mar-13 & Grab & PCB-1221 & U & 05 & $\mathrm{tg} / \mathrm{L}$ \\
\hline 021 & 18-Mar-13 & Grab & PCB-1232 & U & 0.5 & $\mathrm{ug} / \mathrm{L}$ \\
\hline 021 & $18-\mathrm{Mar}-13$ & Grab & PCB- 1242 & $\mathrm{UJ}$ & 05 & $\mathrm{ug} / \mathrm{L}$ \\
\hline 021 & 18-Маг-13 & Grab & PCB-1248 & $\mathrm{U}$ & 0.5 & $\mathrm{ug} / \mathrm{L}$ \\
\hline 021 & 18-Mar-13 & Grab & PCB-1254 & U & 0.5 & $u g / L$ \\
\hline 021 & $18-\mathrm{Mar}-13$ & Grab & PCB- 1260 & I & 0.088 & $\mathrm{ug} / \mathrm{L}$ \\
\hline 021 & 18-Маг-13 & Grab & PCB- 1262 & II & 0.5 & $\mathrm{ug} / \mathrm{L}$ \\
\hline 021 & $18 . \mathrm{Mar}-13$ & Grab & PCB- 1268 & U & 0.5 & ug/l. \\
\hline
\end{tabular}




\section{Major Outfall 021 \\ (continued)}

\begin{tabular}{|c|c|c|c|c|c|c|}
\hline Location & Date Collected & Sample type & Parameter & & Result & Units \\
\hline 021 & 18-Мar-13 & Composite & SuspSolids & & 22.5 & $\mathrm{mg} / \mathrm{l}$ \\
\hline 021 & 18-Mar-13 & Compositc & Surfactants & & 0.207 & $\mathrm{mg} / \mathrm{L}$ \\
\hline 021 & 18-Mar-13 & Composite & KjeldahlN & & 0.648 & $\mathrm{mg} / \mathrm{L}$ \\
\hline 021 & $18-\mathrm{Mar}-13$ & Composite & $\mathrm{NO} 3 / \mathrm{NO} 2 \mathrm{asN}$ & & 0.537 & $\mathrm{mg} / \mathrm{L}$ \\
\hline 021 & 18-Мar-13 & Composite & $N(C a \mid c T o t)$ & & 1.18 & $\mathrm{mg} / \mathrm{l}$ \\
\hline 021 & 18-Mar-I3 & Composite & $\Lambda g$ & $<$ & 0.0002 & $\mathrm{mg} / \mathrm{l}$ \\
\hline 021 & 18-Mar-13 & Compositc & $\mathrm{Al}$ & & 1.26 & $\mathrm{mg} / \mathrm{L}$ \\
\hline 021 & 18-Mar-13 & Composite & As & $<$ & 0.002 & $\mathrm{mg} / \mathrm{L}$ \\
\hline 021 & 18-Mar-13 & Composite & $B$ & $<$ & 0.1 & $\mathrm{mg} / \mathrm{l}$ \\
\hline $02 \downarrow$ & $18-M a r-13$ & Composite & $\mathrm{Ba}$ & & 0.0599 & $\mathrm{mg} / \mathrm{L}$ \\
\hline 021 & 18-Mar-13 & Composite & $\mathrm{Be}$ & $<$ & 0.0002 & $\mathrm{mg} / \mathrm{L}$ \\
\hline 021 & 18-Mar-13 & Composite & $\mathrm{Ca}$ & & 30.3 & $\mathrm{mg} / \mathrm{l}$ \\
\hline 021 & 18-Mar-13 & Composite & $\mathrm{Cd}$ & $<$ & 0.0002 & $\mathrm{mg} / \mathrm{L}$ \\
\hline 021 & 18-Mar-13 & Composite & $\mathrm{Co}$ & & 0.000996 & $\mathrm{mg} / \mathrm{L}$ \\
\hline 021 & 18-Mar-13 & Composite & $\mathrm{Cr}$ & & 000477 & $\mathrm{mg} / \mathrm{L}$ \\
\hline 021 & 18-Маг-13 & Composite & $\mathrm{Cu}$ & & 0.0218 & $\mathrm{mg} / \mathrm{L}$ \\
\hline 02 I & 18-Mar-13 & Composite & $\mathrm{Fe}$ & & 1.62 & $\mathrm{mg} / \mathrm{L}$ \\
\hline 021 & $18-$ Mar- 13 & Composite & $\mathrm{Hg}$ & & 0.0761 & $u g / L$ \\
\hline 021 & 18-Mar-13 & Composite & $\mathrm{K}$ & & 2.23 & $\mathrm{mg} / \mathrm{L}$ \\
\hline 02 I & $18-\mathrm{Mar}-13$ & Composite & $\mathrm{Li}$ & $<$ & 0.01 & $\mathrm{mg} / \mathrm{L}$ \\
\hline 021 & $18-$ Mar-13 & Composite & $\mathrm{Mg}$ & & 5.45 & $\mathrm{mg} / \mathrm{I}$ \\
\hline 021 & 18-Маг-13 & Composite & $\mathrm{Mn}$ & & 0.153 & $\mathrm{mg} / \mathrm{l}$. \\
\hline 021 & 18-Mar-13 & Composite & Mo & & 0.00955 & $\mathrm{mg} / \mathrm{L}$ \\
\hline 021 & $18-$ Mar- 13 & Composite & $\mathrm{Na}$ & & 48.9 & $\mathrm{mg} / \mathrm{L}$ \\
\hline 021 & $18-M a r-13$ & Composite & $\mathrm{Nb}$ & $<$ & 0.2 & $\mathrm{mg} / \mathrm{I}$ \\
\hline 021 & 18-Мат-13 & Composite & $\mathrm{Ni}$ & & 0.0028 & $\mathrm{mg} / \mathrm{L}$ \\
\hline 021 & 18-Mar-13 & Composite & $P$ & $<$ & 0.5 & $\mathrm{mg} / \mathrm{l}$. \\
\hline 021 & 18-Mar-13 & Composite & $\mathrm{Pb}$ & & 0.00698 & $\mathrm{mg} / \mathrm{L}$ \\
\hline 021 & 18-Mar-13 & Composite & $\mathrm{S}$ & & 5.46 & $\mathrm{mg} / \mathrm{L}$ \\
\hline 021 & 18-Mar-13 & Composite & Sb & $<$ & 0.001 & $\mathrm{mg} / \mathrm{L}$ \\
\hline 021 & I8-Mar-। 3 & Composite & $\mathrm{Se}$ & $<$ & 0.004 & $\mathrm{mg} / \mathrm{L}$ \\
\hline 021 & | 8-Mar-13 & Composite & $\mathrm{Sr}$ & & 0.0704 & $\mathrm{mg} / \mathrm{L}$ \\
\hline 021 & 18-Мar-13 & Composite & Th & $<$ & 0.0002 & $\mathrm{mg} / \mathrm{L}$ \\
\hline 021 & $18-\mathrm{Mar}-13$ & Composite & $\mathrm{Ti}$ & $<$ & 0.05 & $\mathrm{mg} / \mathrm{L}$ \\
\hline 021 & 18-Mar-13 & Composite & $\mathrm{Tl}$ & $<$ & 0.0002 & $\mathrm{mg} / \mathrm{L}$ \\
\hline 021 & 18-Mar-13 & Composite & v & $<$ & 0.02 & $\mathrm{mg} / \mathrm{I}$ \\
\hline 021 & 18-Mar-13 & Composite & $\mathrm{Zn}$ & & 0.0883 & $\mathrm{mg} / \mathrm{L}$ \\
\hline 021 & 18 -Мar-13 & Composite & $\mathrm{Zr}$ & $<$ & 0.2 & $\mathrm{mg} / \mathrm{L}$ \\
\hline
\end{tabular}




\section{Major Outfall 021 \\ (continued)}

\begin{tabular}{|c|c|c|c|c|c|c|}
\hline Location & Date Collected & Sample type & Parameter & & Resulı & Units \\
\hline 021 & $18-\mathrm{Mar}-13$ & Composite & Alpha & & 1.8 & $\mathrm{pCi} / \mathrm{L}$ \\
\hline 02$]$ & | 8-Mar-13 & Composite & Beta & & 9.6 & $\mathrm{pCi} / \mathrm{L}$ \\
\hline 021 & 18-Mar-13 & Composite & [] & & 0.0025 & $\mathrm{mg} / \mathrm{L}$ \\
\hline 021 & $18-$ Mar- $^{13}$ & Composite & $\mathrm{U}-234$ & $<$ & 0.005 & w1 $\%$ \\
\hline 021 & 18-Mar-13 & Composite & U $235 \%$ & & 1.74 & $w 1 \%$ \\
\hline 021 & 18-Мar-13 & Composite & U-236 & $<$ & 0.005 & w1 $\%$ \\
\hline 021 & $18-\mathrm{Mar}-13$ & Composite & U-238 & & 98.3 & $\mathbf{w 1} \%$ \\
\hline 021 & $18-\mathrm{Mar}-13$ & Composite & U. 234 & & 0.96 & $\mathrm{pCi} / \mathrm{L}$. \\
\hline 021 & $18-$ Мar-I3 & Composite & U J-235 & & 0014 & $p C^{\prime} / \mathrm{L}$ \\
\hline $02 \mathrm{l}$ & $18-M a r-13$ & Composite & U-236 & & 0.00001 & $\mathrm{pCi} / \mathrm{L}$. \\
\hline 021 & I8-Mar-13 & Composite & U-238 & & 0.72 & $\mathrm{pCi} / \mathrm{L}$ \\
\hline 021 & 18-Mar-13 & Composite & $\mathrm{PCB}$ & $\mathrm{U}$ & 0.47 & $\mathrm{ng} / \mathrm{L}$ \\
\hline 021 & 18-Mar-13 & Composite & PCB-1016 & U & 0.47 & $\mathrm{ug} / \mathrm{L}$ \\
\hline 021 & 18-Mar-13 & Composite & PCB-1221 & $\mathrm{U}$ & 0.47 & $\mathrm{ug} / \mathrm{L}$ \\
\hline 021 & 18-Mar-13 & Composite & РСB-1232 & $\mathrm{U}$ & 0.47 & $\mathrm{ug} / \mathrm{L}$ \\
\hline 021 & 18-Mar-13 & Composite & PCB- 1242 & U & 0.47 & $\mathrm{ug} / \mathrm{L}$. \\
\hline 021 & $18-M a r-13$ & Composite & PCB-1248 & $\mathrm{U}$ & 0.47 & $\mathrm{ug} / \mathrm{L}$ \\
\hline 021 & 18-Mar-13 & Composite & PCB-1254 & $\mathrm{U}$ & 0.47 & $\mathrm{ug} / \mathrm{L}$ \\
\hline 021 & 18-Mar-13 & Composite & PCB- 1260 & $\mathrm{U}$ & 0.47 & $\mathrm{ug} / \mathrm{L}$. \\
\hline 021 & 18-Mar-13 & Composite & РСВ-1262 & II & 0.47 & $\mathrm{ug} / \mathrm{L}$ \\
\hline 021 & 18-Mar-13 & Composite & PCB- 1268 & $\mathrm{U}$ & 0.47 & $\mathrm{ug} / \mathrm{L}$ \\
\hline
\end{tabular}




\section{Major Outfall 109}

\begin{tabular}{|c|c|c|c|c|c|c|}
\hline location & Date Collected & Sample Type & Parameter & & Result & Units \\
\hline 109 & 18-Mar-13 & Field & $\mathrm{pH}$ & & 7.7 & Std Units \\
\hline 109 & 18-Mar-13 & Grab & Es coli & & 63 & $\mathrm{col} / 100 \mathrm{ml}$ \\
\hline 109 & $18-M a r-13$ & Grab & HexExMatl & $<$ & 6 & $\mathrm{mg} / \mathrm{L}$ \\
\hline 109 & 18-Mar-13 & Grab & SuspSolids & & 126 & $\mathrm{mg} / \mathrm{l}$ \\
\hline 109 & $18-$ Мar- 13 & Grab & Surfactants & & 0.29 & $\mathrm{mg} / \mathrm{L}$ \\
\hline 109 & 18-Mar-13 & Grab & Kjeldah|N & & 0.872 & $\mathrm{mg} / \mathrm{L}$ \\
\hline 109 & 18 -Mar-13 & Grab & $\mathrm{NO} 3 / \mathrm{NO} 2 \mathrm{asN}$ & & 0.429 & $\mathrm{mg} / \mathrm{L}$ \\
\hline 109 & 18-Mar-13 & Grab & $N($ Calctot $)$ & & 13 & $\mathrm{mg} / \mathrm{L}$ \\
\hline 109 & 18-Mar-13 & Grab & $\wedge \mathrm{g}$ & $<$ & 00002 & $\mathrm{mg} / \mathrm{L}$ \\
\hline 109 & $18-$ Mar-13 & Grab & $\mathrm{Al}$ & & 162 & $\mathrm{mg} / \mathrm{L}$ \\
\hline 109 & 18-Mar-13 & Grab & $\Lambda \mathrm{s}$ & $<$ & 0002 & $\mathrm{mg} / \mathrm{L}$ \\
\hline 109 & 18-Mar-13 & Grab & $\mathrm{B}$ & $<$ & 0.1 & $\mathrm{mg} / \mathrm{L}$ \\
\hline 109 & 18-Mar-13 & Grab & $\mathrm{Ba}$ & & 00451 & $m g / L$ \\
\hline 109 & 18-Mar- 13 & Grab & $\mathrm{Be}$ & $<$ & 0.0002 & $\mathrm{mg} / \mathrm{L}$ \\
\hline 109 & I8-Mar-13 & Grab & Ca & & 21 & $\mathrm{mg} / \mathrm{L}$ \\
\hline 109 & $18-M a r-13$ & Grab & $\mathrm{Cd}$ & $<$ & 0.0002 & $\mathrm{mg} / \mathrm{L}$ \\
\hline 109 & $18-$ Mar- 13 & Grab & $\mathrm{Co}$ & & 0.000784 & $m g / L$ \\
\hline 109 & 18-Mar-13 & Grab & $\mathrm{Cr}$ & & 000507 & $\mathrm{mg} / \mathrm{L}$ \\
\hline 109 & 18-Mar-13 & Grab & $\mathrm{Cu}$ & & 0.0129 & $\mathrm{mg} / \mathrm{L}$ \\
\hline 109 & 18 -Маг-13 & Grab & $\mathrm{Fe}$ & & 2.01 & $\mathrm{mg} / \mathrm{L}$ \\
\hline 109 & 18-Mar-13 & Grab & $\mathrm{Hg}$ & & 0.0865 & $\mathrm{ug} / \mathrm{L}$ \\
\hline 109 & 18-Mar-13 & Grab & $\mathrm{K}$ & $<$ & 2 & $\mathrm{mg} / \mathrm{L}$. \\
\hline 109 & 18-Mar-13 & Grab & $\mathrm{I}, \mathrm{i}$ & $<$ & 0.01 & $\mathrm{mg} / \mathrm{L}$ \\
\hline 109 & 18-Mar-13 & Grab & $\mathrm{Mg}$ & & 5.26 & $\mathrm{mg} / \mathrm{L}$ \\
\hline 109 & 18-Mar-13 & Grab & $\mathrm{Mn}$ & & 0.109 & $\mathrm{mg} / \mathrm{l}$ \\
\hline 109 & 18-Mar-13 & Grab & Mo & & 0.000596 & $\mathrm{mg} / \mathrm{L}$. \\
\hline 109 & 18-Mar-13 & Grab & $\mathrm{Na}$ & & 54.5 & $\mathrm{mg} / \mathrm{l}$ \\
\hline 109 & 18-Mar-13 & Grab & $\mathrm{Nb}$ & $<$ & 0.2 & $\mathrm{mg} / \mathrm{L}$ \\
\hline 109 & 18 -Mar- 13 & Grab & $\mathrm{Ni}$ & & 0.00269 & $\mathrm{~m} g / \mathrm{L}$ \\
\hline 109 & $18-M a r-13$ & Grab & $\mathrm{P}$ & $<$ & 0.5 & $\mathrm{mg} / \mathrm{L}$ \\
\hline 109 & 18-Mar-13 & Grab & $\mathrm{Pb}$ & & 0.00493 & $\mathrm{mg} / \mathrm{L}$ \\
\hline 109 & $18-M a r-13$ & Grab & $\mathrm{S}$ & & 3.9 & $\mathrm{mg} / \mathrm{L}$ \\
\hline 109 & $18-$ Mar-13 & Grab & $\mathrm{Sb}$ & & 0.00102 & $\mathrm{mg} / \mathrm{L}$ \\
\hline 109 & $18-$ Mar- 13 & Grab & $\mathrm{Se}$ & $<$ & 0.004 & $\mathrm{mg} / \mathrm{L}$ \\
\hline 109 & 18-Mar-13 & Grab & $\mathrm{Sr}$ & & 0.0427 & $\mathrm{mg} / \mathrm{l}$ \\
\hline 109 & 18-Mar-13 & Grab & Th & $<$ & 0.0002 & $\mathrm{mg} / \mathrm{L}$ \\
\hline 109 & 18-Mar-13 & Grab & Ti & & 0.0719 & $\mathrm{mg} / \mathrm{L}$ \\
\hline 109 & 18-Mar-13 & Grab & $\mathrm{T} \mid$ & $<$ & 0.0002 & $\mathrm{mg} / \mathrm{l}$ \\
\hline 109 & $18-\mathrm{Mar}-13$ & Grab & V & $<$ & 0.02 & $\mathrm{mg} / \mathrm{L}$ \\
\hline 109 & 18-Mar-13 & Grab & $\mathrm{Zn}$ & & 0.126 & $\mathrm{mg} / \mathrm{L}$ \\
\hline 109 & $18-M a r-13$ & Grab & $\mathrm{Zr}$ & $<$ & 0.2 & $\mathrm{mg} / \mathrm{L}$ \\
\hline
\end{tabular}




\section{Major Outfall 109 \\ (continued)}

\begin{tabular}{|c|c|c|c|c|c|c|}
\hline Localion & Date Collected & Sample Type & Рaranreter & & Result & Units \\
\hline 109 & 18-Маг-13 & Grab & U & & 0.000756 & $\mathrm{mg} / \mathrm{L}$ \\
\hline 109 & 18-Мат-13 & Grab & Alpha & & 18 & $\mathrm{pCi} / \mathrm{L}$ \\
\hline 109 & 18-Mar-13 & Grab & Beta & & 17 & $\mathrm{pCi} / \mathrm{L}$ \\
\hline 109 & 18-Mar-13 & Grab & U -234 & & $\mathrm{~N} / \mathrm{A}$ & wt \% \\
\hline 109 & $18-M a r-13$ & Grab & U235\% & & $\mathrm{N} / \mathrm{A}$ & w1 \% \\
\hline 109 & 18-Мar-13 & Grab & $\mathrm{U}-236$ & & $\mathrm{~N} / \mathrm{A}$ & w1 \% \\
\hline 109 & 18-Mar-13 & Grab & U-238 & & N/A & wt $\%$ \\
\hline 109 & $18-M a r-13$ & Grab & U-234 & & 8.7 & $\mathrm{pCi} / \mathrm{L}$ \\
\hline 109 & 18-Mar-13 & Grab & $\mathrm{U}-235$ & & 0.28 & $\mathrm{pCi} / \mathrm{L}$ \\
\hline 109 & I 8-Mar-I3 & Grab & $\mathrm{U}-236$ & & 0.12 & $\mathrm{pCi} / \mathrm{L}$ \\
\hline 109 & 18-Mar-13 & Grab & $\mathrm{U}-238$ & & 0.32 & $\mathrm{pCi} / \mathrm{L}$ \\
\hline 109 & 18-Mar-13 & Grab & PCB & $\mathrm{U}$ & 0.46 & $\mathrm{ug} / \mathrm{L}$ \\
\hline 109 & 18-Mar-13 & Girab & PCB-1016 & U & 046 & $\mathrm{Lg} / \mathrm{L}$ \\
\hline 109 & | 8-Mar-13 & Grab & PCB-1221 & $\mathrm{U}$ & 0.46 & $\mathrm{ug} / \mathrm{L}$ \\
\hline 109 & 18-Mar-13 & Grab & PCB- 1232 & $\mathrm{U}$ & 046 & $\mathrm{II} / \mathrm{L}$ \\
\hline 109 & 18-Mar-13 & Grab & PCB- 1242 & $\mathrm{U}$ & 0.46 & $\mathrm{ug} / \mathrm{L}$ \\
\hline 109 & $18-M a r-13$ & Grab & PCB-1248 & U & 0.46 & $\mathrm{ug} / \mathrm{L}$. \\
\hline 109 & 18-Mar-13 & Grab & PCB-1254 & $\mathrm{U}$ & 046 & $\mathrm{ug} / \mathrm{L}$ \\
\hline 109 & 18-Mar-13 & Grab & PCB- 1260 & $\mathrm{U}$ & 0.46 & $11 \mathrm{~g} / \mathrm{L}$ \\
\hline 109 & $18-M a r-13$ & Grab & РСB-1262 & $\mathrm{U}$ & 0.46 & $110 / \mathrm{L}$ \\
\hline 109 & 18-Mar-13 & Grab & PCB-1268 & $\mathrm{U}$ & 0.46 & $11 \mathrm{~g} / \mathrm{L}$ \\
\hline
\end{tabular}




\section{Major Outfall 109 \\ (continued)}

\begin{tabular}{|c|c|c|c|c|c|c|}
\hline Location & Date Collected & Sample Type & Parameter & & Result & Units \\
\hline 109 & | 8-Mar- 3 & Composite & SuspSolids & & 35.5 & $\mathrm{mg} / \mathrm{l}$ \\
\hline 109 & $18-\operatorname{Mar}-13$ & Composite & Surfactants & & 0239 & $\mathrm{mg} / \mathrm{l}$. \\
\hline 109 & 18-Mar-13 & Composite & KjeldahlN & & 0.693 & $\mathrm{mg} / \mathrm{L}$ \\
\hline 109 & 18-Mar-13 & Composite & $\mathrm{NO} 3 / \mathrm{NO} 2 \mathrm{asN}$ & & 0.505 & $\mathrm{mg} / \mathrm{L}$ \\
\hline 109 & 18-Mar-13 & Conposite & $\mathrm{N}($ CalcTot $)$ & & 1.2 & $\mathrm{mg} / \mathrm{L}$ \\
\hline 109 & 18-Mar-13 & Composite & $\mathrm{Ag}$ & $<$ & 0.0002 & $\mathrm{mg} / \mathrm{L}$ \\
\hline 109 & | 8-Mar-1 3 & Composite & $\mathrm{Al}$ & & 1.37 & $\mathrm{mg} / \mathrm{l}$. \\
\hline 109 & 18-Mar-13 & Composite & As & $<$ & 0.002 & $\mathrm{mg} / \mathrm{L}$ \\
\hline 109 & 18-Маг-13 & Compositc & B & $<$ & 0.1 & $\mathrm{mg} / \mathrm{L}$ \\
\hline 109 & 18 -Mar-13 & Composite & $\mathrm{Ba}$ & & 0.0617 & $\mathrm{mg} / \mathrm{L}$. \\
\hline 109 & 18-Mar-13 & Composite & $\mathrm{Be}$ & $<$ & 0.0002 & $\mathrm{mg} / \mathrm{L}$ \\
\hline 109 & 18-Mar-13 & Composite & $\mathrm{Ca}$ & & 35.3 & $\mathrm{mg} / \mathrm{L}$ \\
\hline 109 & 18-Мar-13 & Composile & $\mathrm{Cd}$ & & 0.00073 & $\mathrm{mg} / \mathrm{L}$ \\
\hline 109 & 18-Mar-I3 & Compositc & Co & & 0.00108 & $\mathrm{mg} / \mathrm{L}$ \\
\hline 109 & 18-Mar-I3 & Composile & $\mathrm{Cr}$ & & 0.00439 & $\mathrm{mg} / \mathrm{I}$, \\
\hline 109 & 18-Mar-13 & Composite & $\mathrm{Cu}$ & & 0.012 & $\mathrm{mg} / \mathrm{L}$ \\
\hline 109 & $18-M a r-13$ & Conposite & $\mathrm{ree}$ & & 2.26 & $\mathrm{mg} / \mathrm{L}$ \\
\hline 109 & 18-Mar-13 & Composite & $\mathrm{Hg}$ & & 0.0589 & ug/L \\
\hline 109 & 18-Mar-13 & Composite & $\mathrm{K}$ & & 2.24 & $\mathrm{mg} / \mathrm{L}$ \\
\hline 109 & 18-Mar-13 & Composite & $\mathrm{Li}$ & $<$ & 0.01 & $\mathrm{mg} / \mathrm{L}$ \\
\hline 109 & 18-Мar-13 & Composite & $\mathrm{Mg}$ & & 7.83 & $\mathrm{mg} / \mathrm{L}$ \\
\hline 109 & 18-Mar-13 & Compositc & $\mathrm{Mn}$ & & 0.198 & $\mathrm{mg} / \mathrm{L}$ \\
\hline 109 & $18-$ Mar-13 & Composite & Mo & & 0.000956 & Ing/L \\
\hline 109 & 18-Mar-13 & Compositc & $\mathrm{Na}$ & & 61.9 & $\mathrm{mg} / \mathrm{L}$ \\
\hline 109 & 18-Mar-13 & Compositc & $\mathrm{Nb}$ & $<$ & 0.2 & $\mathrm{mg} / \mathrm{l}$. \\
\hline 109 & $18-\mathrm{Mar}-13$ & Composite & $\mathrm{Ni}$ & & 0.0034 & $\mathrm{mg} / \mathrm{l}$. \\
\hline 109 & 18-Mar-13 & Composite & $p$ & $<$ & 0.5 & $\mathrm{mg} / \mathrm{L}$ \\
\hline 109 & 18-Mar-13 & Composite & $\mathrm{Pb}$ & & 0.00502 & $\mathrm{mg} / \mathrm{L}$ \\
\hline 109 & 18-Mar-13 & Composite & $\mathrm{S}$ & & 6.53 & $\mathrm{rng} / \mathrm{L}$ \\
\hline 109 & 18-Mar-13 & Composite & Sb & & 0.00606 & $\mathrm{mg} / \mathrm{L}$ \\
\hline 109 & 18-Mar-I3 & Composile & Se & $<$ & 0,004 & $\mathrm{mg} / \mathrm{l}$ \\
\hline 109 & 18-Mar-13 & Composite & $\mathrm{Sr}$ & & 0.0838 & $\mathrm{mg} / \mathrm{L}$ \\
\hline 109 & 18 -Маг-13 & Composile & Th & $<$ & 0.0002 & $\mathrm{mg} / \mathrm{l}$. \\
\hline 109 & 18-Мar-13 & Composite & Tî & & 0.0668 & $\mathrm{mg} / \mathrm{L}$ \\
\hline 109 & 18-Mar-13 & Composite & $\mathrm{Tl}$ & $<$ & 0.0002 & $\mathrm{mg} / \mathrm{L}$ \\
\hline 109 & 18-Mar-13 & Composite & $\mathrm{v}$ & $<$ & 0.02 & $\mathrm{mg} / \mathrm{L}$ \\
\hline 109 & 18-Mar-13 & Composite & $\mathrm{Zn}$ & & 0.0942 & $\mathrm{mg} / \mathrm{L}$ \\
\hline 109 & 18-Mar-13 & Composile & $\mathrm{Zr}$ & $<$ & 0.2 & $\mathrm{mg} / \mathrm{l}$. \\
\hline
\end{tabular}




\section{Major Outfall 109 \\ (continued)}

\begin{tabular}{|c|c|c|c|c|c|c|}
\hline Location & Date Collected & Sample Type & Parameter & & Result & Units \\
\hline 109 & | 8-Mar-|3 & Composite & U & & 0.00148 & $\mathrm{mg} / \mathrm{L}$ \\
\hline 109 & 18-Mar-13 & Composite & Alpha & & 8.3 & $\mathrm{pCi} / \mathrm{L}$ \\
\hline 109 & 18-Mar-13 & Composite & Beta & & 4.9 & $\mathrm{pCi} / \mathrm{l}$ \\
\hline 109 & |8-Mar- $\mid 3$ & Composite & U-234 & $<$ & 0.005 & $w t \%$ \\
\hline 109 & 18-Mar-13 & Composite & U $235 \%$ & & 607 & wt \% \\
\hline 109 & 18-Mar-13 & Composite & U-236 & & 0.21 & wt \% \\
\hline 109 & I8-Маг-।3 & Composite & U. 238 & & 93.7 & w1 $\%$ \\
\hline 109 & 18-Mar-13 & Composite & U-235 & & 0.21 & $n \mathrm{Ci} / \mathrm{L}$ \\
\hline 109 & 18-Mar-13 & Composite & U -236 & & 0.012 & $\mathrm{pCi} / \mathrm{L}$ \\
\hline 109 & 18-Mar-13 & Composite & U-238 & & 0.47 & $\mathrm{pCi} / \mathrm{L}$ \\
\hline 109 & 18-Mar-13 & Composite & U -234 & & 4.2 & $\mathrm{pCi} / \mathrm{L}$ \\
\hline 109 & 18-Mar-13 & Composite & PCB & $\mathrm{U}$ & 0.46 & $\mathrm{ug} / \mathrm{L}$ \\
\hline 109 & 18-Мar-13 & Composite & РCB-1016 & $\mathrm{U}$ & 0.46 & $\mathrm{ug} / \mathrm{L}$ \\
\hline 109 & | 8-Мат-13 & Composite & PCB-122I & $\mathrm{U}$ & 0.46 & $\mathrm{ug} / \mathrm{L}$ \\
\hline 109 & 18-Mar-13 & Compositc & PCB- 1232 & $\mathrm{U}$ & 0.46 & $\mathrm{ug} / \mathrm{L}$ \\
\hline 109 & 18-Mar-13 & Composite & PCB-1242 & U & 0.46 & $\mathrm{ug} / \mathrm{L}$ \\
\hline 109 & 18-Mar-13 & Composite & PCB-1248 & $\mathrm{U}$ & 0.46 & $\mathrm{ug} / \mathrm{L}$. \\
\hline 109 & 18-Mar-13 & Composite & PCB- 1254 & $\mathrm{U}$ & 0.46 & $\mathrm{ug} / \mathrm{L}$ \\
\hline 109 & 18-Mar-13 & Composite & PC'B- 1260 & $\mathrm{U}$ & 0.46 & $\mathrm{ug} / \mathrm{L}$ \\
\hline 109 & 18-Mar-13 & Composite & PCB- 1262 & $\mathrm{U}$ & 0.46 & $u g / L$ \\
\hline 109 & 18-Mar-13 & Composite & РCB- 1268 & $\mathrm{U}$ & 0.46 & $\mathrm{ug} / \mathrm{L}$ \\
\hline
\end{tabular}




\section{Major Outfall 200}

\begin{tabular}{|c|c|c|c|c|c|c|}
\hline Localion & Date Colleeted & Sample Type & Parameter & & Result & Units \\
\hline 200 & $20 \mid 3 / 03 / 18$ & Field & $\mathrm{pH}$ & & 7.7 & Std Units \\
\hline 200 & $2013 / 03 / 18$ & Grab & Es coli & & 700 & $\mathrm{col} / 100 \mathrm{ml}$ \\
\hline 200 & $2013 / 03 / 18$ & Grab & HexExMatl & $<$ & 6 & $\mathrm{mg} / \mathrm{L}$ \\
\hline 200 & $2013 / 03 / 18$ & Grab & KjeldahlN & & 0.837 & $\mathrm{mg} / \mathrm{l}$. \\
\hline 200 & $2013 / 03 / 18$ & Grab & $\mathrm{NO} 3 / \mathrm{NO} 2 \mathrm{as} \mathrm{N}$ & & 329 & $\mathrm{mlg} / \mathrm{L}$ \\
\hline 200 & $2013 / 03 / 18$ & Grab & $N($ CalcTot $)$ & & 4.13 & $\mathrm{mg} / \mathrm{l}$. \\
\hline 200 & $2013 / 03 / 18$ & Grab & SuspSolids & & 55 & $\mathrm{tng} / \mathrm{L}$ \\
\hline 200 & $2013 / 03 / 18$ & Girab & $\mathrm{Ag}$ & $<$ & 0.0002 & $\mathrm{mg} / \mathrm{l}$. \\
\hline 200 & $20 \mid 3 / 03 / 18$ & Grab & $\mathrm{Al}$ & & 1.06 & $\mathrm{mg} / \mathrm{l}$ \\
\hline 200 & $20\lrcorner 3 / 03 / / 8$ & Grab & As & $<$ & 0.002 & $\mathrm{mg} / \mathrm{l}$ \\
\hline 200 & $2013 / 03 / 18$ & Grab & $\mathrm{B}$ & $<$ & 0.1 & $\mathrm{mg} / \mathrm{l}$ \\
\hline 200 & $2013 / 03 / 18$ & Grab & $\mathrm{Ba}$ & & 0.0692 & $\mathrm{mg} / \mathrm{L}$ \\
\hline 200 & $2013 / 03 / 18$ & Grab & $\mathrm{Be}$ & $<$ & 0.0002 & $\mathrm{mg} / \mathrm{L}$ \\
\hline 200 & $2013 / 03 / 18$ & Grab & $\mathrm{Ca}$ & & 51.7 & $\mathrm{mg} / \mathrm{L}$ \\
\hline 200 & $2013 / 03 / 18$ & Grab & $\mathrm{Cd}$ & & 000142 & $\mathrm{mg} / \mathrm{L}$ \\
\hline 200 & $2013 / 03 / 18$ & Grab & $\mathrm{Co}$ & & 0.00154 & $\mathrm{mg} / \mathrm{L}$ \\
\hline 200 & $2013 / 03 / 18$ & Grab & $\mathrm{Cr}$ & & 0.0039 & $\mathrm{mg} / \mathrm{L}$ \\
\hline 200 & $2013 / 03 / 18$ & Grab & $\mathrm{Cu}$ & & 0.044 & $\mathrm{mg} / \mathrm{L}$ \\
\hline 200 & $2013 / 03 / 18$ & Grab & $\mathrm{Fe}$ & & 1.59 & $\mathrm{mg} / \mathrm{L}$ \\
\hline 200 & $2013 / 03 / 18$ & Grab & $\mathrm{Hg}$ & & 0.00257 & $\mathrm{mg} / \mathrm{L}$ \\
\hline 200 & $2013 / 03 / 18$ & Grab & $\mathrm{K}$ & & 2.7 & $\mathrm{mg} / \mathrm{L}$ \\
\hline 200 & $2013 / 03 / 18$ & Grab & $\mathrm{Li}$ & & 0.0244 & $\mathrm{mg} / \mathrm{l}$. \\
\hline 200 & $2013 / 03 / 18$ & Grab & $\mathrm{Mg}$ & & 11.5 & $\mathrm{mg} / \mathrm{l}$. \\
\hline 200 & $2013 / 03 / 18$ & Grab & $\mathrm{Mn}$ & & 0.245 & $\mathrm{mg} / \mathrm{l}$. \\
\hline 200 & $2013 / 03 / 18$ & Grab & Mo & & 00038 & $\mathrm{mg} / \mathrm{l}$. \\
\hline 200 & $2013 / 03 / 18$ & Grab & $\mathrm{Na}$ & & 27 & $\mathrm{mg} / \mathrm{L}$ \\
\hline 200 & $2013 / 03 / 18$ & Grab & $\mathrm{Nb}$ & $<$ & 0.2 & $\mathrm{mg} / \mathrm{L}$ \\
\hline 200 & $2013 / 03 / 18$ & Grab & $\mathrm{Ni}$ & & 000468 & $\mathrm{mg} / \mathrm{L}$ \\
\hline 200 & $2013 / 03 / 18$ & Grab & $P$ & $<$ & 0.5 & $\mathrm{mg} / \mathrm{L}$ \\
\hline 200 & $2013 / 03 / 18$ & Grab & $\mathrm{Pb}$ & & 0.0102 & $\mathrm{mg} / \mathrm{L}$ \\
\hline 200 & $2013 / 03 / 18$ & Grab & $\mathrm{S}$ & & 8.98 & $\mathrm{mg} / 1$. \\
\hline 200 & $2013 / 03 / 18$ & Grab & $\mathrm{Sb}$ & $<$ & 0.001 & $\mathrm{mg} / \mathrm{L}$ \\
\hline 200 & $2013 / 03 / 18$ & Grab & $\mathrm{Se}$ & $<$ & 0.004 & $\mathrm{mg} / \mathrm{L}$ \\
\hline 200 & $2013 / 03 / 18$ & Grab & $\mathrm{Sr}$ & & 0.126 & $\mathbf{m g} /]$ \\
\hline 200 & $2013 / 03 / 18$ & Grab & Th & $<$ & 0.0002 & $\mathbf{m g} / \mathrm{L}$ \\
\hline 200 & $2013 / 03 / 18$ & Grab & $\mathrm{Ti}$ & $<$ & 0.05 & $\mathrm{mg} / \mathrm{L}$ \\
\hline 200 & $2013 / 03 / 18$ & Grab & $\mathrm{TI}$ & $<$ & 0.0002 & $\mathrm{mg} / \mathrm{L}$ \\
\hline 200 & $2013 / 03 / 18$ & Grab & v & $<$ & 0.02 & $\mathrm{mg} / \mathrm{L}$ \\
\hline 200 & $2013 / 03 / 18$ & Grab & 7. n & & 0.179 & $\mathrm{mg} / \mathrm{L}$ \\
\hline 200 & $2013 / 03 / 18$ & Grab & $\mathrm{Zr}$ & $<$ & 0.2 & $\mathrm{mg} / \mathrm{L}$ \\
\hline 200 & $2013 / 03 / 18$ & Grab & Alpha & & 18 & $\mathrm{pCi} / \mathrm{L}$ \\
\hline 200 & $2013 / 03 / 18$ & Grab & Beta & & 12 & $\mathrm{pCi} / \mathrm{L}$ \\
\hline 200 & $2013 / 03 / 18$ & Grab & $\mathrm{U}$ & & 0.0585 & $\mathrm{mg} / \mathrm{l}$ \\
\hline 200 & $2013 / 03 / 18$ & Grab & $\mathrm{U}-234$ & $<$ & 0.005 & wt $\%$ \\
\hline 200 & $2013 / 03 / 18$ & Grab & U235\% & & 0.249 & wt \% \\
\hline 200 & $2013 / 03 / 18$ & Grab & U-236 & & 0.00876 & $w 1 \%$ \\
\hline 200 & $2013 / 03 / 18$ & Grab & U-238 & & 99.7 & wt $\%$ \\
\hline 200 & $2013 / 03 / 18$ & Grab & U-234 & & 4 & $\mathrm{pCi} / \mathrm{L}$ \\
\hline 200 & $2013 / 03 / 18$ & Grab & U-235 & & 0.4 & $\mathrm{pCi} / \mathrm{L}$ \\
\hline 200 & $2013 / 03 / 18$ & Grab & U-236 & & 0.14 & $\mathrm{pCi} / \mathrm{L}$ \\
\hline 200 & $2013 / 03 / 18$ & Grab & U-238 & & 18 & $\mathrm{pCl} / \mathrm{L}$ \\
\hline
\end{tabular}




\section{Major Outfall 200 \\ (continued)}

\begin{tabular}{|c|c|c|c|c|c|c|}
\hline Location & Date Collected & Sample Type & Parameter & & Result & Units \\
\hline 200 & $20 / 3 / 03 / 18$ & Composite & Surlactants & & 0.125 & $\mathrm{mg} / \mathrm{L}$ \\
\hline 200 & $20 / 3 / 03 / 18$ & Composite & SuspSolids & & 15.5 & $\mathrm{mg} / \mathrm{L}$ \\
\hline 200 & $2013 / 03 / 18$ & Composite & KjeldahiN & & 0.797 & $\mathbf{m g} / \mathrm{l}$ \\
\hline 200 & $20 / 3 / 03 / 18$ & Composite & $\mathrm{NO} 3 / \mathrm{NO} 2 \mathrm{asN}$ & & 2.05 & $\mathrm{mg} / \mathrm{L}$ \\
\hline 200 & $2013 / 03 / 18$ & Composite & $N(C a l c T o t)$ & & 285 & $\mathrm{mg} / \mathrm{l}$ \\
\hline 200 & $2013 / 03 / 18$ & Composite & $\mathrm{Ag}$ & $<$ & 0.0002 & $\mathrm{mg} / \mathrm{L}$ \\
\hline 200 & $2013 / 03 / 18$ & Composite & $\mathrm{Al}$ & & 0.558 & $\operatorname{lng} / \mathrm{l}$. \\
\hline 200 & $2013 / 03 / 18$ & Composite & As & $<$ & 0.002 & $m \mathrm{~g} / \mathrm{L}$ \\
\hline 200 & $2013 / 03 / 18$ & Composite & B & $<$ & 0.1 & $\mathrm{mg} / \mathrm{l}$. \\
\hline 200 & $2013 / 03 / 18$ & Composite & $\mathrm{Ba}$ & & 0.0391 & $\mathrm{mg} / \mathrm{L}$ \\
\hline 200 & $2013 / 03 / 18$ & Composite & $\mathrm{Be}$ & $<$ & 0.0002 & $\mathrm{Ing} / \mathrm{l}$. \\
\hline 200 & $2013 / 03 / 18$ & Composite & $\mathrm{Ca}$ & & 30 & $\mathrm{mg} / \mathrm{L}$ \\
\hline 200 & $2013 / 03 / 18$ & Composite & $\mathrm{Cd}$ & & 0.000532 & $\operatorname{lng} / \mathrm{l}$. \\
\hline 200 & $2013 / 03 / 18$ & Composite & Co & & 0.00053 & $\mathrm{mg} / \mathrm{L}$ \\
\hline 200 & $2013 / 03 / 18$ & Composite & $\mathrm{C}_{\mathrm{r}}$ & & 0.0029 & $\mathrm{mg} / \mathrm{L}$ \\
\hline 200 & $2013 / 03 / 18$ & Composite & CiI & & 0.0148 & $\mathrm{mg} / \mathrm{L}$ \\
\hline 200 & $2013 / 03 / 18$ & Composite & $\mathrm{Fe}$ & & 0.717 & $\mathrm{mg} / \mathrm{L}$ \\
\hline 200 & $2013 / 03 / 18$ & Composite & $\mathrm{Hg}$ & & 0.000503 & $\operatorname{lng} / \mathrm{I}$ \\
\hline 200 & $2013 / 03 / 18$ & Composite & $\mathrm{K}$ & $<$ & 2 & $\mathrm{mg} / \mathrm{L}$ \\
\hline 200 & $2013 / 03 / 18$ & Composite & $\mathrm{L} . \mathrm{i}$ & & 0.0336 & $\mathrm{mg} / \mathrm{L}$ \\
\hline 200 & $2013 / 03 / 18$ & Composite & $\mathrm{Mg}$ & & 6.07 & $\operatorname{lng} / \mathrm{L}$ \\
\hline 200 & $2013 / 03 / 18$ & Composite & $\mathrm{Mn}$ & & 0.068 & $\mathrm{mg} / \mathrm{L}$ \\
\hline 200 & $2013 / 03 / 18$ & Composite & Mo & & $0.002 ! 6$ & $\mathrm{mg} / \mathrm{L}$ \\
\hline 200 & $2013 / 03 / 18$ & Composite & $\mathrm{Na}$ & & 264 & $\mathrm{mg} / \mathrm{L}$ \\
\hline 200 & $2013 / 03 / 18$ & Composite & $\mathrm{Nb}$ & $<$ & 0.2 & $\mathrm{mg} / \mathrm{l}$ \\
\hline 200 & $2013 / 03 / 18$ & Composite & $\mathrm{Ni}$ & & 0.00205 & $\mathrm{mg} / \mathrm{l}$. \\
\hline 200 & $2013 / 03 / 18$ & Composite & $\mathrm{P}$ & $<$ & 0.5 & $\mathrm{mo} / \mathrm{L}$ \\
\hline 200 & $2013 / 03 / 18$ & Composite & $\mathrm{Pb}$ & & 0.00671 & $\mathrm{mg} / \mathrm{L}$ \\
\hline 200 & $2013 / 03 / 18$ & Composite & $S$ & & 6.3 & $\mathrm{mg} / \mathrm{l}$ \\
\hline 200 & $2013 / 03 / 18$ & Composite & $\mathrm{Sb}$ & $<$ & 0.001 & $\mathrm{mg} / \mathrm{L}$ \\
\hline 200 & $2013 / 03 / 18$ & Composite & $\mathrm{Se}$ & $<$ & 0.004 & $\mathrm{mg} / \mathrm{L}$ \\
\hline 200 & $2013 / 03 / 18$ & Composite & $\mathrm{Sr}$ & & 00756 & $\mathrm{mg} / \mathrm{l}$ \\
\hline 200 & $20 / 3 / 03 / / 8$ & Composite & $\mathrm{Tl}$ & $<$ & 0.0002 & $\mathrm{mg} / \mathrm{L}$ \\
\hline 200 & $2013 / 03 / 18$ & Composite & $\mathrm{Ti}$ & $<$ & 0.05 & $\mathrm{mg} / \mathrm{L}$ \\
\hline 200 & $2013 / 03 / 18$ & Composite & $\mathrm{Tl}$ & $<$ & 0.0002 & $\mathbf{m g} / \mathrm{l}$. \\
\hline 200 & $2013 / 03 / / 8$ & Composite & v & $<$ & 0.02 & $\mathrm{mg} / \mathrm{L}$ \\
\hline 200 & $2013 / 03 / 18$ & Composite & $\mathrm{Zn}$ & & 0.0752 & $\mathrm{mg} / \mathrm{L}$ \\
\hline 200 & $2013 / 03 / 18$ & Composite & $\mathrm{Zr}$ & $<$ & 0.2 & $\mathrm{mg} / \mathrm{L}$ \\
\hline
\end{tabular}




\section{Major Outfall 200 (continued)}

\begin{tabular}{|c|c|c|c|c|c|c|}
\hline Location & Date Collected & Sample Type & Parameter & & Resulı & Units \\
\hline 200 & $2013 / 03 / 18$ & Composite & Alpha & & 18 & $\mathrm{pCi} / \mathrm{L}$ \\
\hline 200 & $20 \mid 3 / 03 / 18$ & Composite & Bela & & 16 & $\mathrm{pCi} / \mathrm{L}$ \\
\hline 200 & $2013 / 03 / 18$ & Composite & $\mathrm{U}$ & & 0.0317 & $\mathrm{mg} / \mathrm{L}$ \\
\hline 200 & $20 / 3 / 03 / 18$ & Composite & $\mathrm{U}-234$ & $<$ & 0.005 & wi $\%$ \\
\hline 200 & $2013 / 03 / 18$ & Composite & U235\% & & 0.287 & wt $\%$ \\
\hline 200 & $2013 / 03 / 18$ & Composite & U-236 & & 0.0136 & wt $\%$ \\
\hline 200 & $2013 / 03 / 18$ & Composite & U-238 & & 99.7 & $w t \%$ \\
\hline 200 & $2013 / 03 / 18$ & Composite & $\mathrm{U}-234$ & & 2.9 & $\mathrm{pCi} / \mathrm{L}$ \\
\hline 200 & $2013 / 03 / 18$ & C'omposite & $\mathrm{J} J-235$ & & 0.25 & $\mathrm{pCi} / \mathrm{L}$ \\
\hline 200 & $2013 / 03 / 18$ & Composite & U-238 & & 11 & $\mathrm{pCi} / \mathrm{L}$ \\
\hline 200 & $2013 / 03 / 18$ & Composite & $\mathrm{PCB}$ & $\mathrm{U}$ & 0.46 & $\mathrm{ug} / \mathrm{L}$ \\
\hline 200 & $2013 / 03 / 18$ & Composile & PCB-1016 & $\mathrm{U}$ & 0.46 & $\mathrm{ug} / \mathrm{L}$ \\
\hline 200 & $2013 / 03 / 18$ & Composite & PCB-122] & $\mathrm{U}$ & 0.46 & $\mathrm{ug} / \mathrm{L}$ \\
\hline 200 & $2013 / 03 / 18$ & Composite & PCB- 1232 & U & 0.46 & $\mathrm{ug} / \mathrm{L}$. \\
\hline 200 & $2013 / 03 / 18$ & Composite & PCB- 1242 & U & 0.46 & $\mathrm{ug} / \mathrm{L}$ \\
\hline 200 & $2013 / 03 / 18$ & Composite & PCB- 1248 & U & 0.46 & $\mathrm{ug} / \mathrm{L}$ \\
\hline 200 & $2013 / 03 / 18$ & Composite & PCB- 1254 & [] & 0.46 & $\mathrm{ug} / \mathrm{L}$ \\
\hline 200 & $2013 / 03 / 18$ & Composite & PCB- 1260 & U & 0.46 & $\mathrm{ug} / \mathrm{L}$ \\
\hline 200 & $2013 / 03 / 18$ & Composite & РСB- 1262 & U & 0.46 & $\mathrm{ug} / \mathrm{L}$ \\
\hline 200 & $2013 / 03 / 18$ & Compositc & РCB- 1268 & U & 0.46 & $\mathrm{ug} / \mathrm{L}$ \\
\hline
\end{tabular}

